Revised manuscript. Article published in J. Org. Chem. 2015, 80, 3321-3331 (DOI: $10.1021 / \mathrm{jo502912m)}$

\title{
$\mathrm{Pd}$-catalyzed directed ortho-C-H alkenylation of
}

\section{phenylalanine derivatives}

Alfonso García-Rubia, ${ }^{\dagger}$ Eduardo Laga, ${ }^{\dagger}$, Carlos Cativiela,$^{\star}$ Esteban P. Urriolabeitia, ${ }^{\star}$ Ramón GómezArrayás $^{\dagger *}$ and Juan C. Carretero ${ }^{\dagger *}$

† Departamento de Química Orgánica, Facultad de Ciencias, Universidad Autónoma de Madrid, Cantoblanco, 28049 Madrid, Spain. *Instituto de Síntesis Química y Catálisis Homogénea (ISQCH), CSIC-Universidad de Zaragoza, Pedro Cerbuna 12, E-50009 Zaragoza, Spain.

ramon.gomez@uam.es; juancarlos.carretero@uam.es<smiles>CCOC(=O)C(Cc1ccccc1)N(C)S(C)(=O)=O</smiles><smiles>CO[C@H](c1ccccc1)[C@@H](C)N(C)S(C)(=O)=O</smiles>

$\left(\left[\mathrm{F}^{+}\right]=N\right.$-fluoro-2,4,6-trimethylpyridinium triflate $)$
CEwg (1-2 equiv) $\mathrm{Pd}(\mathrm{OAc})_{2}(10 \mathrm{~mol} \%)$

$\left[\mathrm{F}^{+}\right]$(3.0 equiv)

$\mathrm{AcOH}, 110^{\circ} \mathrm{C}$

20 examples
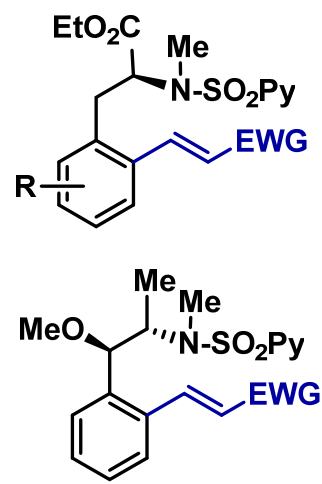

ABSTRACT: A practical Pd-catalyzed ortho-olefination of enantioenriched $N$-( $\left.\mathrm{SO}_{2} \mathrm{Py}\right)$-protected arylalanine and norephedrine derivatives with electron deficient alkenes has been developed using $N$-fluoro2,4,6-trimethylpyridinium triflate as the terminal oxidant. The reaction occurs efficiently with excellent mono-substitution selectivity and without loss of enantiopurity. This cross coupling proved to be broad 
Revised manuscript. Article published in J. Org. Chem. 2015, 80, 3321-3331 (DOI: $10.1021 / j 0502912 \mathrm{~m}$ )

in scope, tolerating a variety of steric and electronic changes to both coupling partners. Removal of the directing group under mild conditions provides access to optically active tetrahydroisoquinoline-3carboxylic acid derivatives (Tics) with good diastereocontrol and with very small erosion of enantiomeric purity.

Keywords: Palladium; C-H Olefination; Phenylalanine; Norephedrine; 2-Pyridylsulfonyl; Tetrahydroisoquinoline.

The direct functionalization of $\mathrm{C}-\mathrm{H}$ bonds is an area of great interest because of its potential to streamline synthetic routes. ${ }^{1}$ The use of directing groups has become a common strategy to address the challenge of achieving predictable selectivity in substrates that lack strong steric or electronic biases or to override the inherent substrate biasing elements. ${ }^{1-3}$

Coordination-directed $\mathrm{C}-\mathrm{H}$ bond functionalization of nitrogen-containing compounds, such as $\alpha$ amino acids, is particularly attractive given their prevalence in natural products and therapeutic agents. ${ }^{4-}$ ${ }^{8}$ Moreover, the incorporation of flexible functional handles into the amino acid skeleton provide products that offer high versatility as building blocks for their diversification into valuable heterocyclic frameworks. ${ }^{9}$ Despite their significance, catalytic metal-controlled direct functionalization of $\alpha$-amino acids remain underdeveloped. ${ }^{5,7,8}$ An important challenge is to find a suitable directing function capable of preventing interference by the potentially coordinating proximal $\alpha$-ester group and overcoming the increased steric restriction caused by the already installed $\alpha$-stereocenter. An added requirement of such methods is mild functionalization/deprotection conditions tolerant of sensitive functional groups and the easily racemizable $\alpha$-stereocenter. Most of the reported procedures are suited for $\mathrm{N}$-protected $\mathrm{NH}$ amino acid derivatives, ${ }^{5-8}$ due to the participation of active metal amide intermediate complexes, whereas catalytic direct functionalization of $\mathrm{N}$-substituted substrates (instead of $\mathrm{NH}$-amino acid 
Revised manuscript. Article published in J. Org. Chem. 2015, 80, 3321-3331 (DOI: $10.1021 / j 0502912 \mathrm{~m}$ )

derivatives) remains an important ongoing challenge. Additionally, controlling mono- vs. disubstitution selectivity is often problematic in this type of processes. ${ }^{10}$

Within this field, the $\mathrm{Pd}^{\mathrm{II}}$-catalyzed oxidative coupling between arenes and olefins (Fujiwara-Moritani reaction) is arguably one of the most important reactions in this field. ${ }^{11,12}$ Recently, our group introduced the use of the $N$-(2-pyridyl)sulfonyl $\left(\mathrm{SO}_{2} \mathrm{Py}\right)$ moeity as an easily installable and removable protecting/directing group in a variety of Pd-catalyzed $\mathrm{C}-\mathrm{H}$ functionalization reactions. ${ }^{13}$ In particular, it showed a high directing ability, as well as structural flexibility, in the $\mathrm{Pd}^{\mathrm{II}}$-catalyzed aryl $\mathrm{C}-\mathrm{H}$ ortho alkenylation of anilines, benzylamines and phenethylamines with electron-defficient alkenes. ${ }^{13 \mathrm{c}}$ Subsequently, $\mathrm{Yu}$ and co-workers demonstrated the suitability of this $\mathrm{N}-\mathrm{SO}_{2} \mathrm{Py}$-strategy for $\mathrm{Pd}^{\mathrm{II}}$ catalyzed intramolecular $\mathrm{C}-\mathrm{H}$ amination of phenethylamine derivatives, including optically active phenylalanine derivatives. ${ }^{7 \mathrm{~h}}$ Herein, we report a practical and efficient $\mathrm{Pd}^{\mathrm{II}}$-catalyzed mono-orthoolefination of enantioenriched phenylalanine derivatives. The use of the $\mathrm{N}$-SO $\mathrm{S}_{2}$ Py-protecting group allows for facile deprotection and subsequent cyclization via aza-Michael addition of the free amino group to the acrylate moiety to form disubstituted tetrahydroisoquinolines with good diastereocontrol.

On the basis of the knowledge acquired in our previous contribution, ${ }^{13 \mathrm{c}}$ the enantiomerically pure $N$ methyl- $N-\left(\mathrm{SO}_{2} \mathrm{Py}\right)$-protected phenylalanine ethyl ester derivative $(S)-\mathbf{1}$ was probed for its potential in the olefination reaction with butyl acrylate (2.0 equiv) using $\mathrm{Pd}(\mathrm{OAc})_{2}$ as catalyst $(10 \mathrm{~mol} \%)$ and $\mathrm{N}$ fluoro-2,4,6-trimethylpyridium triflate $\left[\mathrm{F}^{+}\right]$as stoichiometric oxidant, ${ }^{14}$ at $110{ }^{\circ} \mathrm{C}$ for $18 \mathrm{~h}$. The results of this optimization study are shown in Table 1 . The reaction in DCE showed poor conversion (40\%, entry 1), while changing the solvent to $\mathrm{AcOH}$ resulted in a slightly higher conversion (entry 2). Despite the low conversions, the reactions were very clean and in the latter case the desired ortho-olefination product $(S)-2$ was isolated in $45 \%$ yield. It is important to note that the stereochemical information present in the starting phenylalanine derivative $(S)$-1 was preserved in the olefin-bearing product $(S)-\mathbf{2}$ with minimal erosion in the enantiomeric purity $(97 \%$ ee, entry 2 ; the starting $(S)-1$ was prepared from 
Revised manuscript. Article published in J. Org. Chem. 2015, 80, 3321-3331 (DOI: $10.1021 / j 0502912 \mathrm{~m}$ )

commercially available L-phenylalanine of $\geq 98 \%$ ee). The use of hexafluoroisopropanol (HFIP), a solvent that proved to be highly effective in $\mathrm{C}-\mathrm{H}$ functionalization of $\alpha$-amino acid derivatives, ${ }^{8 \mathrm{i}}$ resulted in a boost in conversion up to $94 \%$. However, this increase in reactivity ( $83 \%$ yield of the desired product 2 ) came at the cost of a loss of enantiomeric purity ( $88 \%$ ee, entry 3$)$.

Table 1. Optimization of reaction conditions. ${ }^{a}$

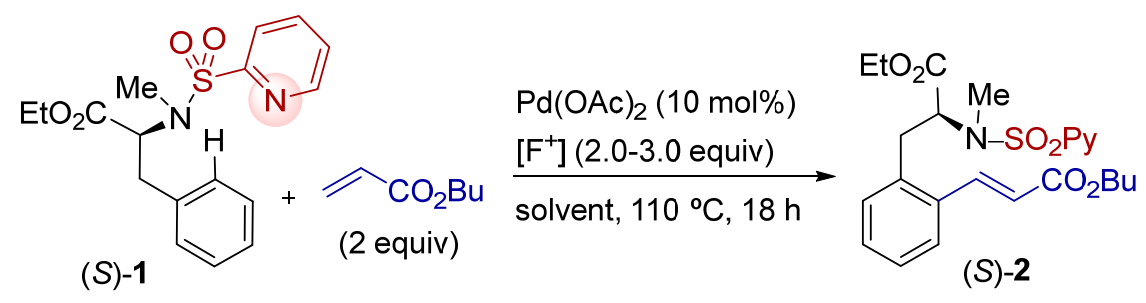

$\left[\mathrm{F}^{+}\right]=N$-fluoro-2,4,6-trimethylpyridinium triflate

\begin{tabular}{lllll}
\hline Entry & Solvent & {$[\mathrm{F}+]$ (equiv) } & Conversion $(\%)^{b}$ & ee $(\%)^{c}$ \\
\hline 1 & DCE & 2.0 & 40 & - \\
2 & $\mathrm{AcOH}$ & 2.0 & $53(45)^{d}$ & 97 \\
3 & $\mathrm{HFIP}$ & 2.0 & $94(83)^{d}$ & 88 \\
4 & $\mathrm{AcOH}$ & 2.5 & 72 & - \\
5 & $\mathrm{AcOH}$ & 3.0 & $>98$ & - \\
$6^{e}$ & $\mathrm{AcOH}$ & 3.0 & $>98(84)^{d}$ & 97
\end{tabular}

${ }^{a}$ Conditions: (-)-1 (0.15 mmol), $\mathrm{Pd}(\mathrm{OAc}) 2(0.015 \mathrm{mmol}),\left[\mathrm{F}^{+}\right](0.45 \mathrm{mmol}), \mathrm{AcOH}(1 \mathrm{~mL})$ at $110{ }^{\circ} \mathrm{C}$, butyl acrylate $(0.30 \mathrm{mmol}) .{ }^{b} \mathrm{By}{ }^{1} \mathrm{H}$ NMR from the crude mixture, measuring the relative ratio of 2 to starting 1 by integration of charasterictic, baseline separated signals. ${ }^{c}$ Enantiomeric excess of $(S)-\mathbf{2}$, dtermined by chiral stationary phase HPLC. ${ }^{d}$ In parenthesis, isolated yield after chromatography. ${ }^{e}$ Reaction time: 6 hours.

To our satisfaction, the solution to the problem of low reactivity without compromising the stereochemical integrity was found by increasing the amount of the oxidant. Thus, the model reaction of 1a with butyl acrylate in $\mathrm{AcOH}$ in the presence of 2.5 equivalents of $\left[\mathrm{F}^{+}\right]$led to $72 \%$ conversion of 2 (entry 4), while further increase of $\left[\mathrm{F}^{+}\right]$to 3.0 equivalents allowed for complete conversion (entry 5). 
Revised manuscript. Article published in J. Org. Chem. 2015, 80, 3321-3331 (DOI: 10.1021/jo502912m)

Furthermore, under these latter conditions complete conversion was observed after only 6 hours, affording the acrylate 2 in good yield (84\%) without appreciable loss of enantiopurity (97\% ee, entry 6). It should be noted that, in contrast to literature precedents, ${ }^{11}$ in which mono-olefination selectivity is often difficult to control, complete mono-substitution selectivity was observed under our reaction conditions.

After the optimal reaction parameters had been established, the effect of electronic and structural variations on the alkene was studied. For that purpose, a variety of monosubstituted electrophilic alkenes were surveyed in the reaction with the parent substrate $\mathbf{1}$ (Scheme 1). For this study, racemic ( \pm -1 was used in most cases. Not only acrylates (products $\mathbf{2}$ and $\mathbf{3}$ ), but also dimethyl vinyl phosphonate (4), phenyl vinyl sulfone (5) and phenyl vinyl sulfonate (6) coupled efficiently with 1 to give the corresponding ortho-alkenylated products with excellent regioselectivity, mono-substitution selectivity and $E$-stereoselectivity in synthetically useful yields (68-84\%). Other carbonyl derivatives such as methyl vinyl ketone (7) or acrolein (8) resulted less efficient coupling partners (43\% and 25\% yield, respectively). Interestingly, styrene derivatives bearing electron-withdrawing substituents (pentafluoro, $\mathrm{NO}_{2}$ or $\mathrm{CF}_{3}$ ) at the phenyl ring were found to be also capable reactants for the alkenylation reaction, affording the corresponding products in acceptable yields (products 9-11, 37-56\% yield). Unfortunately, styrene itself provided poor reactivity $(<10 \%$ conversion, not shown). We confirmed again that the alkenylation reaction takes place with no appreciable racemization, as demonstrated in the reaction with phenyl vinyl sulfone (product $(S)-5,79 \%$ yield, $>98 \%$ ee). 
Revised manuscript. Article published in J. Org. Chem. 2015, 80, 3321-3331 (DOI: $10.1021 / j 0502912 \mathrm{~m}$ )

Scheme 1. Scope with regard to the olefin coupling partner.
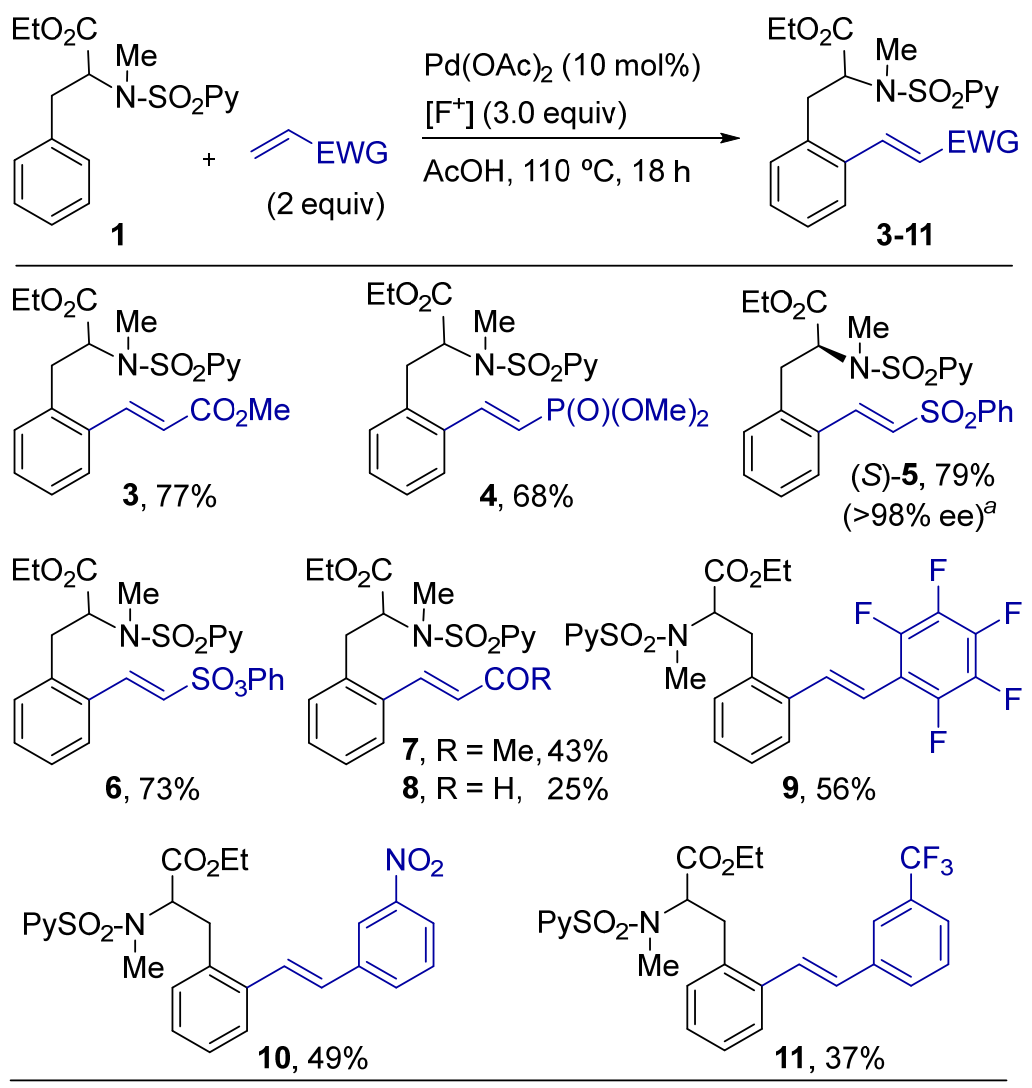

${ }^{a}$ The starting $(S)$-1 was prepared from commercially available L-phenylalanine of $\geq 98 \%$ ee. $\left[\mathrm{F}^{+}\right]=N$ fluoro-2,4,6-trimethylpyridinium triflate. $\mathrm{EWG}=$ electron-withdrawing functional group.

Encouraged by the good tolerance of our catalyst system towards different functional groups, structurally diverse phenylalanine derivatives were subjected to the catalytic olefination with butyl acrylate in order to evaluate the scope and limitations with regard to the aromatic substitution of the amino acid counterpart. This scope study was limited by the number of commercially available arylalanines. The results are presented in Scheme 2. Both electron-rich such as (OMe and Me), and electron-deficient groups $(\mathrm{F}, \mathrm{Cl}, \mathrm{Br})$ were tolerated, although substrates with electron-releasing groups are generally more reactive. Thus, the $p$-OMe product 12 (a derivative of tyrosine) was obtained with 
Revised manuscript. Article published in J. Org. Chem. 2015, 80, 3321-3331 (DOI: $10.1021 / j 0502912 \mathrm{~m}$ )

$71 \%$ yield, whereas no reactivity was observed in the substrate holding a $p-\mathrm{NO}_{2}$ group $(<10 \%$ conversion, result not shown in Scheme 2). Nevertheless, a number of mono ortho-olefinated phenylalanine derivatives were isolated in acceptable yields (24-72\%). It should be noted that in the case of $p$ - $\mathrm{Cl}$ or $p$ - $\mathrm{Br}$ substituted substrates, the incomplete conversions and the difficulty in the complete chromatographic separation of the products from the starting material and trace amount of unidentified byproducts resulted in low yields upon isolation (products 15 and 16, 24\% and 29\% yield, respectively). ${ }^{15}$ Despite these shortcomings, the partial tolerance to halogen-containing substrates is useful for subsequent product derivatization since it provides orthogonal reactivity relative to the $\mathrm{Pd}^{0}$ catalyzed cross-coupling chemistry.

When a substrate having blocked the meta position was employed, as in the case of the 2-naphthyl derivative, the reaction occurred at a sterically more accessible site (C-3') to avoid the steric congestion (product 18, 52\% yield). However, meta-fluoro-substitution caused the formation of a mixture of the two ortho-olefination regioisomers (17a and $\mathbf{1 7 b}$ were isolated with 72\% yield in a 43:57 relative ratio), probably arising from the low steric discrimination of the two ortho positions by the small fluoro substituent. The 1-naphthyl derivative underwent clean olefination at the available C-2' position (19, $69 \%$ yield). 
Revised manuscript. Article published in J. Org. Chem. 2015, 80, 3321-3331 (DOI: $10.1021 / j 0502912 \mathrm{~m}$ )

Scheme 2. Scope with regard to the substitution at the aromatic ring. ${ }^{a}$
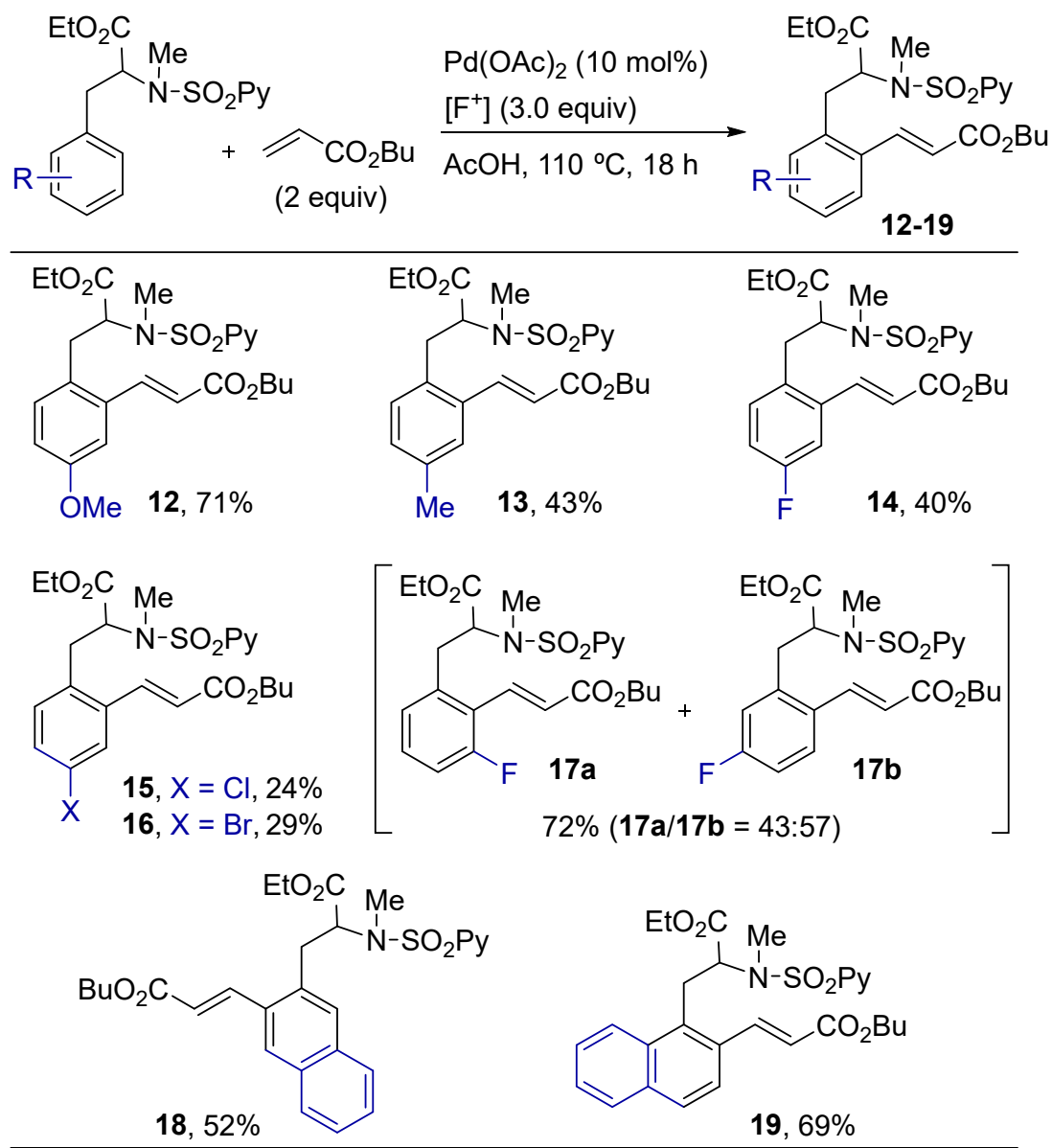

${ }^{a}\left[\mathrm{~F}^{+}\right]=N$-fluoro-2,4,6-trimethylpyridinium triflate.

Notably, this method can be further extended to chiral enantiomerically pure $\alpha, \beta$-disubstituted phenethylamines, such as the norephedrine derivative 20 (Scheme 3). Compound 20 was smoothly alkenylated under the standard reaction conditions with butyl acrylate or phenyl vinyl sulfone (1.0 equiv) to give the corresponding products in good yields (74\%-78\%) without epimerization, according to ${ }^{1} \mathrm{H}$ NMR. 
Revised manuscript. Article published in J. Org. Chem. 2015, 80, 3321-3331 (DOI: 10.1021/jo502912m)

Scheme 3. Extension to norephedrine derivatives<smiles>[R]C=CC(=O)OC(C)C(C)C(OC)c1ccccc1</smiles>

$(-)-20$

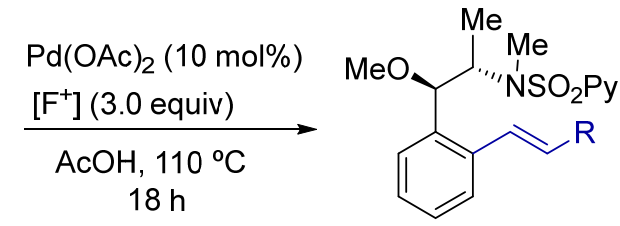

$(-)-21, \mathrm{R}=\mathrm{CO}_{2} \mathrm{Bu}, 74 \%$

$(-)-22, \mathrm{R}=\mathrm{SO}_{2} \mathrm{Ph}, 78 \%$

The easy reductive removal of the $N$-(2-pyridyl)sulfonyl protecting/directing group under smooth conditions ( $\mathrm{Zn}$ powder, 1:1 THF/sat. aq $\mathrm{NH}_{4} \mathrm{Cl}, 65^{\circ} \mathrm{C}$ ) without affecting the sensitive acrylate moiety enabled the direct access to valuable 1,2,3,4-tetrahydroisoquinoline-3-carboxylic acid (Tic) ${ }^{16}$ skeletons in synthetically useful yields (Scheme 4). For example, $N$-deprotection of product $( \pm$ )-2 simultaneously triggers the cyclization of the free amine under the reaction conditions to afford $\mathbf{2 3}$ in $71 \%$ yield and good diastereoselectivity $($ anti/syn $=80: 20) .{ }^{17}$ When the enantiomerically pure product $(S)-5(>98 \%$ ee $)$ was submitted to identical conditions, the same $N$-deprotection/intramolecular aza-Michael sequence was produced, affording the corresponding bicyclic product 24 in similar yield (74\%) and diastereocontrol (anti/syn $=80: 20)$, albeit with a small degree of racemization ( $95 \%$ ee in the major anti diastereomer).

Scheme 4. $N$-deprotection/cyclization to afford tetrahydroisoquinolines
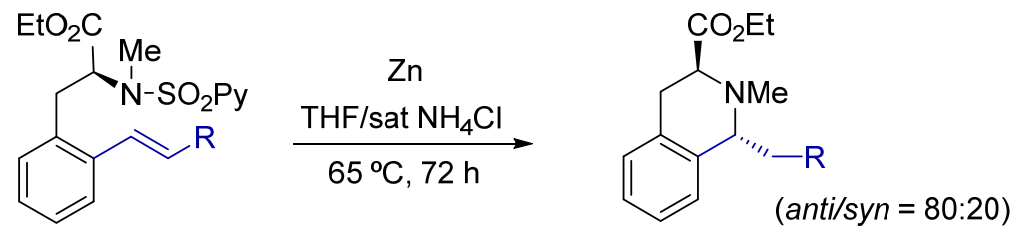
$( \pm)-2, \mathrm{R}=\mathrm{CO}_{2} \mathrm{Bu}$
$( \pm)-23, \mathrm{R}=\mathrm{CO}_{2} \mathrm{Bu}, 71 \%$
(S) $-5, \mathrm{R}=\mathrm{SO}_{2} \mathrm{Ph}$
(+)-24, $\mathrm{R}=\mathrm{SO}_{2} \mathrm{Ph}, 74 \%$ (95\% ee, anti) 
Revised manuscript. Article published in J. Org. Chem. 2015, 80, 3321-3331 (DOI: 10.1021/jo502912m)

In conclusion, we have demonstrated that the directing ability of the easily removable $N$-(2pyridyl)sulfonyl group can be extended to the $\mathrm{Pd}^{\mathrm{II}}$-catalyzed direct ortho-olefination of $N$-substituted phenylalanine and norephedrine derivatives in acceptable yields and with preservation of enantiopurity of the starting substrate. This protocol features good structural versatility in both alkene and nitrogen arene components. The smooth $N$-deprotection without affecting the acrylate moiety enables rapid access to enantioenriched tetrahydroisoquinoline derivatives.

\section{EXPERIMENTAL SECTION}

\subsection{General methods}

Solvents were used as received from commercial sources. They were not distilled nor subjected to additional purification. All reactions were carried out without special protections against air and moisture. Column liquid chromatography was performed on silica gel $\left(70-230\right.$ mesh). The ${ }^{1} \mathrm{H},{ }^{13} \mathrm{C}\left\{{ }^{1} \mathrm{H}\right\}$, ${ }^{19} \mathrm{~F}$ and ${ }^{31} \mathrm{P}\left\{{ }^{1} \mathrm{H}\right\}$ NMR spectra were recorded in $\mathrm{CDCl}_{3}$ solutions at $25{ }^{\circ} \mathrm{C}$ on 300,400 or $500 \mathrm{MHz}$ spectrometers $(\delta$, ppm; $J, \mathrm{~Hz}) ;{ }^{1} \mathrm{H}$ and ${ }^{13} \mathrm{C}\left\{{ }^{1} \mathrm{H}\right\}$ spectra were referenced using the solvent signal as internal standard, while ${ }^{19} \mathrm{~F}$ and ${ }^{31} \mathrm{P}$ NMR spectra were referenced to $\mathrm{CFCl}_{3}$ and $\mathrm{H}_{3} \mathrm{PO}_{4}(85 \%)$, respectively. HRMS and ESI (ESI+) mass spectra were recorded using an MicroToF Q, API-Q-ToF ESI with a mass range from 20 to $3000 \mathrm{~m} / \mathrm{z}$ and mass resolution 15000 (FWHM). HPLC experiments were conducted on an Agilent 1100 instrument, using Daicel Chiralpak IA, AD, AS-H and Chiralcel OJ-H columns as chiral stationary phase. Optical rotations were measured on a polarimeter and they are

reported as follows: $[\alpha]_{\text {wavelenght }}$ temperature $(c$, solvent). Melting points were determined in open-end capillary tubes. 
Revised manuscript. Article published in J. Org. Chem. 2015, 80, 3321-3331 (DOI: $10.1021 / j 0502912 \mathrm{~m}$ )

Synthesis of the starting $N$-(2-pyridyl)sulfonyl phenylalanine ethyl ester derivatives from arylalanines

\section{Step 1: Protection of the carboxylic acid group as ethyl ester}

Typical procedure: synthesis of $(S)$-ethyl 2-amino-3-phenylpropanoate $(S)$-I. To an ice-cooled solution $\left(-5{ }^{\circ} \mathrm{C}\right)$ of $(S)$-phenylalanine $(2.00 \mathrm{~g}, 12.11 \mathrm{mmol}$; $\geq 98 \%$ ee $)$ in ethanol $(10 \mathrm{~mL})$ was added thionyl chloride $(1.32 \mathrm{~mL}, 18.16 \mathrm{mmol})$. The resulting mixture was heated to reflux for 3 hours, it was allowed to reach room temperature and the solvent was evaporated. The solid residue was treated with a sat. aq solution of sodium hydrogen carbonate $(10 \mathrm{~mL})$ and extracted with ethyl acetate $(3 \times 10 \mathrm{~mL})$. The combined organic phase was dried over anhydrous magnesium sulfate, filtered and concentrated to give $(S)-I$ as a clear yellow oil; yield: $1.82 \mathrm{~g}(78 \%)$

${ }^{1} \mathrm{H}$ NMR $\left(300 \mathrm{MHz}, \mathrm{CDCl}_{3}\right): \delta 7.29-7.10(\mathrm{~m}, 5 \mathrm{H}), 4.09(\mathrm{q}, J=7.1 \mathrm{~Hz}, 2 \mathrm{H}), 3.63(\mathrm{t}, \mathrm{br}, J=5.3 \mathrm{~Hz}$, 1H), $3.01(\mathrm{dd}, J=13.5,5.3 \mathrm{~Hz}, 1 \mathrm{H}), 2.81(\mathrm{dd}, J=13.5,7.6 \mathrm{~Hz}, 1 \mathrm{H}), 1.58(\mathrm{~s}, \mathrm{br}, 2 \mathrm{H}), 1.17(\mathrm{t}, J=7.2 \mathrm{~Hz}$, $3 \mathrm{H}) .{ }^{13} \mathrm{C}\left\{{ }^{1} \mathrm{H}\right\} \mathrm{NMR}\left(75 \mathrm{MHz}, \mathrm{CDCl}_{3}\right): \delta 174.7,137.1,129.0,128.2,126.4,60.5,55.5,40.9,13.9$. ESIHRMS: Calcd for $\mathrm{C}_{11} \mathrm{H}_{16} \mathrm{NO}_{2}[\mathrm{M}+\mathrm{H}]^{+}$: 194.1176. Found: 194.1185. $[\alpha]_{\mathrm{D}}^{298}=-7.04\left(c 1.09, \mathrm{CHCl}_{3}\right)$.

( \pm )-Ethyl 2-amino-3-(4-methoxyphenyl)propanoate $[( \pm)-I I]$. Following the typical procedure, thionyl chloride $(445.9 \mu \mathrm{L}, 6.15 \mathrm{mmol})$ reacted with O-methyltyrosine $(800.0 \mathrm{mg}, 4.10 \mathrm{mmol})$ in ethanol (5 mL) to give, after workup, $( \pm)-$ II as a pale yellow oil; yield: $694.4 \mathrm{mg}(76 \%)$.

${ }^{1} \mathrm{H}$ NMR $\left(300 \mathrm{MHz}, \mathrm{CDCl}_{3}\right): \delta 6.96(\mathrm{~d}, J=8.6 \mathrm{~Hz}, 2 \mathrm{H}), 6.68(\mathrm{~d}, J=8.6 \mathrm{~Hz}, 2 \mathrm{H}), 4.00(\mathrm{q}, J=7.2 \mathrm{~Hz}$, 2H), $3.60(\mathrm{~d}, J=1.0 \mathrm{~Hz}, 3 \mathrm{H}), 3.50(\mathrm{dd}, J=7.5,5.5 \mathrm{~Hz}, 1 \mathrm{H}), 2.85(\mathrm{dd}, J=13.6,5.4 \mathrm{~Hz}, 1 \mathrm{H}), 2.67(\mathrm{dd}, J$ $=13.6,7.5 \mathrm{~Hz}, 1 \mathrm{H}), 1.74(\mathrm{~s}, \mathrm{br}, 2 \mathrm{H}), 1.09(\mathrm{t}, J=7.1 \mathrm{~Hz}, 3 \mathrm{H}) .{ }^{13} \mathrm{C}\left\{{ }^{1} \mathrm{H}\right\} \mathrm{NMR}\left(75 \mathrm{MHz}, \mathrm{CDCl}_{3}\right): \delta 174.6$, 158.0, 129.8, 128.8, 113.4, 60.3, 55.4, 54.6, 39.7, 13.7. ESI-HRMS: Calcd for $\mathrm{C}_{12} \mathrm{H}_{18} \mathrm{NO}_{3}[\mathrm{M}+\mathrm{H}]^{+}$: 224.1281. Found: 224.1276. 
Revised manuscript. Article published in J. Org. Chem. 2015, 80, 3321-3331 (DOI: $10.1021 / j 0502912 \mathrm{~m}$ )

( \pm )-Ethyl 2-amino-3-(p-tolyl)propanoate [( \pm -III]. Following the typical procedure, thionyl chloride $(242.9 \mu \mathrm{L}, 3.35 \mathrm{mmol})$ reacted with $p$-methylphenylalanine $(400.0 \mathrm{mg}, 2.23 \mathrm{mmol})$ in ethanol $(10 \mathrm{~mL})$ to give, after workup, $( \pm)-$ III as a pale yellow oil; yield: $461.0 \mathrm{mg}(99 \%)$.

${ }^{1} \mathrm{H}$ NMR (300 MHz, $\left.\mathrm{CDCl}_{3}\right): \delta 7.14-7.01(\mathrm{~m}, 4 \mathrm{H}), 4.15(\mathrm{q}, J=7.2 \mathrm{~Hz}, 2 \mathrm{H}), 3.66(\mathrm{dd}, J=7.8,5.2 \mathrm{~Hz}$, 1H), $3.02(\mathrm{dd}, J=13.5,5.2 \mathrm{~Hz}, 1 \mathrm{H}), 2.80(\mathrm{dd}, J=13.5,7.8 \mathrm{~Hz}, 1 \mathrm{H}), 2.30(\mathrm{~s}, 3 \mathrm{H}), 1.49(\mathrm{~s}, 2 \mathrm{H}), 1.23(\mathrm{t}, J$ $=7.1 \mathrm{~Hz}, 3 \mathrm{H}) .{ }^{13} \mathrm{C}\left\{{ }^{1} \mathrm{H}\right\} \mathrm{NMR}\left(75 \mathrm{MHz}, \mathrm{CDCl}_{3}\right): \delta 175.0,136.2,134.1,129.1,129.1,60.8,55.8,40.6$, 21.0, 14.1. ESI-HRMS: Calcd for $\mathrm{C}_{12} \mathrm{H}_{18} \mathrm{NO}_{2}[\mathrm{M}+\mathrm{H}]^{+}:$208.1332. Found: 208.1327.

( \pm )-Ethyl 2-amino-3-(4-fluorophenyl)propanoate [( $( \pm)-I V]$. Following the typical procedure, thionyl chloride $(475.2 \mu \mathrm{L}, 6.55 \mathrm{mmol})$ reacted with $p$-fluorophenylalanine $(800.0 \mathrm{mg}, 4.37 \mathrm{mmol})$ in ethanol (5 $\mathrm{mL})$ to give, after workup, $( \pm)-\mathbf{I V}$ as a pale yellow oil; yield: $851.0 \mathrm{mg}(92 \%)$.

${ }^{1} \mathrm{H}$ NMR (300 MHz, $\left.\mathrm{CDCl}_{3}\right): \delta 7.11-6.99(\mathrm{~m}, 2 \mathrm{H}), 6.92-6.81(\mathrm{~m}, 2 \mathrm{H}), 4.03(\mathrm{q}, J=7.1 \mathrm{~Hz}, 2 \mathrm{H}), 3.56$ (s, br, 1H), $2.91(\mathrm{dd}, J=13.5,5.3 \mathrm{~Hz}, 1 \mathrm{H}), 2.74(\mathrm{dd}, J=13.6,7.4 \mathrm{~Hz}, 1 \mathrm{H}), 1.51(\mathrm{~s}, \mathrm{br}, 2 \mathrm{H}), 1.11(\mathrm{t}, J=$ $7.1 \mathrm{~Hz}, 3 \mathrm{H}) .{ }^{13} \mathrm{C}\left\{{ }^{1} \mathrm{H}\right\} \mathrm{NMR}\left(75 \mathrm{MHz}, \mathrm{CDCl}_{3}\right): \delta 174.6,161.5\left(\mathrm{~d}, J_{\mathrm{C}-\mathrm{F}}=244.6 \mathrm{~Hz}\right), 132.9\left(\mathrm{~d}, J_{\mathrm{C}-\mathrm{F}}=3.2\right.$ $\mathrm{Hz}), 130.5\left(\mathrm{~d}, J_{\mathrm{C}-\mathrm{F}}=7.9 \mathrm{~Hz}\right), 115.0\left(\mathrm{~d}, J_{\mathrm{C}-\mathrm{F}}=21.2 \mathrm{~Hz}\right), 60.6,55.5,40.0,13.9 . \delta_{\mathrm{F}:}-115.88$. ESI-HRMS Calcd for $\mathrm{C}_{11} \mathrm{H}_{15} \mathrm{FNO}_{2}[\mathrm{M}+\mathrm{H}]^{+}:$212.1081. Found: 212.1080 .

( \pm )-Ethyl 2-amino-3-(4-chlorophenyl)propanoate $[( \pm)-\mathrm{V}]$. Following the typical procedure, thionyl chloride $(545.1 \mu \mathrm{L}, 7.51 \mathrm{mmol})$ reacted with $p$-chlorophenylalanine $(1.00 \mathrm{~g}, 5.01 \mathrm{mmol})$ in ethanol (10 $\mathrm{mL})$ to give, after workup, $( \pm)-\mathbf{V}$ as a pale yellow oil; yield: $1.12 \mathrm{~g}(99 \%)$.

${ }^{1} \mathrm{H}$ NMR (300 MHz, $\left.\mathrm{CDCl}_{3}\right): \delta 7.21(\mathrm{~d}, J=7.9 \mathrm{~Hz}, 2 \mathrm{H}), 7.08(\mathrm{~d}, J=8.0 \mathrm{~Hz}, 2 \mathrm{H}), 4.10(\mathrm{q}, J=7.2 \mathrm{~Hz}$, 2H), $3.62(\mathrm{t}, J=6.9 \mathrm{~Hz}, 1 \mathrm{H}), 2.97(\mathrm{dd}, J=13.4,5.4 \mathrm{~Hz}, 1 \mathrm{H}), 2.78(\mathrm{dd}, J=13.6,7.7 \mathrm{~Hz}, 1 \mathrm{H}), 1.43(\mathrm{~s}$, br, 2H), $1.19(\mathrm{t}, J=7.0 \mathrm{~Hz}, 3 \mathrm{H}) .{ }^{13} \mathrm{C}\left\{{ }^{1} \mathrm{H}\right\} \mathrm{NMR}\left(75 \mathrm{MHz}, \mathrm{CDCl}_{3}\right): \delta 174.7,135.8,132.5,130.6,128.5$, 60.9, 55.6, 40.3, 14.1. ESI-HRMS: Calcd for $\mathrm{C}_{11} \mathrm{H}_{15} \mathrm{ClNO}_{2}[\mathrm{M}+\mathrm{H}]^{+}:$228.0786. Found: 228.0772 . 
Revised manuscript. Article published in J. Org. Chem. 2015, 80, 3321-3331 (DOI: $10.1021 / j 0502912 \mathrm{~m}$ )

( \pm )-Ethyl 2-amino-3-(4-bromophenyl)propanoate $[( \pm)-\mathrm{VI}]$. Following the typical procedure, thionyl chloride $(445.8 \mu \mathrm{L}, 6.14 \mathrm{mmol})$ reacted with $p$-bromophenylalanine $(1.00 \mathrm{~g}, 4.10 \mathrm{mmol})$ in ethanol $(10 \mathrm{~mL})$ to give, after workup, $( \pm)-V I$ as a pale yellow oil; yield: $1.04 \mathrm{~g} \mathrm{(93 \% ).}$

${ }^{1} \mathrm{H}$ NMR (300 MHz, $\left.\mathrm{CDCl}_{3}\right): \delta 7.45-7.34(\mathrm{~m}, 2 \mathrm{H}), 7.13-7.02(\mathrm{~m}, 2 \mathrm{H}), 4.14(\mathrm{q}, J=7.1 \mathrm{~Hz}, 2 \mathrm{H}), 3.65$ $(\mathrm{dd}, J=7.8,5.4 \mathrm{~Hz}), 3.00(\mathrm{dd}, J=13.6,5.4 \mathrm{~Hz}, 1 \mathrm{H}), 2.80(\mathrm{dd}, J=13.6,7.7 \mathrm{~Hz}, 1 \mathrm{H}), 1.43(\mathrm{~s}, 2 \mathrm{H}), 1.22$ $(\mathrm{t}, J=7.1 \mathrm{~Hz}, 3 \mathrm{H}) .{ }^{13} \mathrm{C}\left\{{ }^{1} \mathrm{H}\right\} \mathrm{NMR}\left(75 \mathrm{MHz}, \mathrm{CDCl}_{3}\right): \delta 174.87,136.46,131.62,131.10,120.77,61.07$, 55.73, 40.53, 14.27. ESI-HRMS: Calcd for $\mathrm{C}_{11} \mathrm{H}_{15} \mathrm{BrNO}_{2}[\mathrm{M}+\mathrm{H}]^{+}:$272.0286. Found: 272.0269 .

( \pm -Ethyl 2-amino-3-(3-fluorophenyl)propanoate [( \pm )-VII] Following the typical procedure, thionyl chloride $(207.1 \mu \mathrm{L}, 2.85 \mathrm{mmol})$ reacted with $m$-fluor phenylalanine $(366.4 \mathrm{mg}, 2.00 \mathrm{mmol})$ in ethanol (5 mL) to give, after workup, $( \pm)-$ VII as a pale yellow oil; yield: $333.5 \mathrm{mg}(79 \%)$.

${ }^{1} \mathrm{H}$ NMR $\left(300 \mathrm{MHz}, \mathrm{CDCl}_{3}\right): \delta 8.82(\mathrm{~s}, \mathrm{br}, 2 \mathrm{H}), 7.29(\mathrm{~m}, 1 \mathrm{H}), 7.12(\mathrm{t}, J=8.1 \mathrm{~Hz}, 2 \mathrm{H}), 6.95(\mathrm{t}, J=7.8$ $\mathrm{Hz}, 1 \mathrm{H}), 4.44(\mathrm{~m}, 1 \mathrm{H}), 4.13(\mathrm{~m}, 2 \mathrm{H}), 3.51(\mathrm{~m}, 1 \mathrm{H}), 3.36(\mathrm{~m}, 1 \mathrm{H}), 1.15(\mathrm{t}, J=6.8 \mathrm{~Hz}, 3 \mathrm{H}) .{ }^{13} \mathrm{C}\left\{{ }^{1} \mathrm{H}\right\}$ NMR $\left(75 \mathrm{MHz}, \mathrm{CDCl}_{3}\right): \delta 168.8,162.0\left(\mathrm{~d}, J_{\mathrm{C}-\mathrm{F}}=46.5 \mathrm{~Hz}\right), 136.9\left(\mathrm{~d}, J_{\mathrm{C}-\mathrm{F}}=7.8 \mathrm{~Hz}\right), 130.3\left(\mathrm{~d}, J_{\mathrm{C}-\mathrm{F}}=8.2\right.$ $\mathrm{Hz}), 125.4\left(\mathrm{~d}, J_{\mathrm{C}-\mathrm{F}}=2.9 \mathrm{~Hz}\right), 116.7\left(\mathrm{~d}, J_{\mathrm{C}-\mathrm{F}}=21.4 \mathrm{~Hz}\right), 114.5\left(\mathrm{~d}, J_{\mathrm{C}-\mathrm{F}}=20.9 \mathrm{~Hz}\right), 62.5,54.2,36.0,13.8$. ${ }^{19} \mathrm{~F}\left\{{ }^{1} \mathrm{H}\right\}$ NMR (282 MHz, $\left.\mathrm{CDCl}_{3}\right): \delta$-112.7. ESI-HRMS: Calcd for $\mathrm{C}_{11} \mathrm{H}_{15} \mathrm{FNO}_{2}[\mathrm{M}+\mathrm{H}]^{+}: 212.1081$. Found: 212.1088.

( \pm )-Ethyl 2-amino-3-(naphthalen-1-yl)propanoate $[( \pm)-\mathrm{VIII}]$. Following the typical procedure, thionyl chloride $(207.1 \mu \mathrm{L}, 2.85 \mathrm{mmol})$ reacted with 1-naphtylalanine (430.3 $\mathrm{mg}, 2.00 \mathrm{mmol})$ in ethanol $(5 \mathrm{~mL})$ to give, after workup, ( \pm )-VIII as a pale yellow oil; yield: $340.6 \mathrm{mg}(70 \%)$.

${ }^{1} \mathrm{H}$ NMR $\left(300 \mathrm{MHz}, \mathrm{CDCl}_{3}\right): \delta 8.07(\mathrm{~d}, J=7.9 \mathrm{~Hz}, 1 \mathrm{H}), 7.80(\mathrm{~d}, J=7.6 \mathrm{~Hz}, 1 \mathrm{H}), 7.61(\mathrm{~d}, J=8.1 \mathrm{~Hz}$, 1H), 7.40-7.18 (m, 6H), $4.43(\mathrm{~m}, 1 \mathrm{H}), 3.97(\mathrm{~m}, 1 \mathrm{H}), 3.64(\mathrm{~m}, 3 \mathrm{H}), 0.63(\mathrm{t}, J=7.0 \mathrm{~Hz}, 3 \mathrm{H}) .{ }^{13} \mathrm{C}\left\{{ }^{1} \mathrm{H}\right\}$ NMR (75 MHz, $\left.\mathrm{CDCl}_{3}\right): \delta 169.5,133.8,131.9,131.0,128.7,128.5,128.1,126.5,125.7,125.5,123.6$, 62.1, 53.9, 34.0, 13.3. ESI-HRMS: Calcd for $\mathrm{C}_{15} \mathrm{H}_{18} \mathrm{NO}_{2}[\mathrm{M}+\mathrm{H}]^{+}:$244.1332. Found: 244.1335. 
Revised manuscript. Article published in J. Org. Chem. 2015, 80, 3321-3331 (DOI: $10.1021 / j o 502912 \mathrm{~m}$ )

( \pm )-Ethyl 2-amino-3-(naphthalen-2-yl)propanoate $[( \pm)$-IX]. Following the typical procedure, thionyl chloride $(207.1 \mu \mathrm{L}, 2.85 \mathrm{mmol})$ reacted with 2-naphtylalanine (430.3 $\mathrm{mg}, 2.00 \mathrm{mmol})$ in ethanol ( $5 \mathrm{~mL})$ to give, after workup, $( \pm)-\mathbf{I X}$ as a pale yellow oil; yield: $384.4 \mathrm{mg}(79 \%)$.

${ }^{1} \mathrm{H}$ NMR (300 MHz, $\left.\mathrm{CDCl}_{3}\right): \delta 8.88(\mathrm{~s}, \mathrm{br}, 2 \mathrm{H}), 7.80-7.74(\mathrm{~m}, 4 \mathrm{H}), 7.44-7.39(\mathrm{~m}, 3 \mathrm{H}), 4.52(\mathrm{t}, J=7.1$ $\mathrm{Hz}, 1 \mathrm{H}), 3.97(\mathrm{~m}, 2 \mathrm{H}), 3.67(\mathrm{dd}, J=5.4,14.1 \mathrm{~Hz}, 1 \mathrm{H}), 3.50(\mathrm{dd}, J=7.9,13.9 \mathrm{~Hz}, 1 \mathrm{H}), 0.96(\mathrm{t}, J=7.0$ $\mathrm{Hz}, 3 \mathrm{H}) .{ }^{13} \mathrm{C}\left\{{ }^{1} \mathrm{H}\right\} \mathrm{NMR}\left(75 \mathrm{MHz}, \mathrm{CDCl}_{3}\right): \delta 169.0,133.4,132.6,131.9,128.7,128.3,127.8,127.6$, 127.4, 126.1, 125.8, 62.3, 54.3, 36.6, 13.7. ESI-HRMS: Calcd for $\mathrm{C}_{15} \mathrm{H}_{18} \mathrm{NO}_{2}[\mathrm{M}+\mathrm{H}]^{+}:$244.1332. Found: 244.1338.

\section{Step 2: $N$-sulfonylation reaction}

Typical procedure: synthesis of $(S)$-ethyl 3-phenyl-2-(pyridine-2-sulfonamido)propanoate [(S)X]. To a solution of $(S)-\mathbf{I}(1.82 \mathrm{~g}, 9.43 \mathrm{mmol})$ and pyridine $(2.7 \mathrm{~mL}, 33.96 \mathrm{mmol})$ in dry THF $(20 \mathrm{~mL})$, cooled to $-5{ }^{\circ} \mathrm{C}$ and under Ar atmosphere, was added drop-wise a solution of 2-pyridylsulfonyl chloride $(2.01 \mathrm{~g}, 11.32 \mathrm{mmol})$ in dry THF $(5 \mathrm{~mL})$. The resulting mixture was allowed to reach room temperature and stirred for further $18 \mathrm{~h}$ before it was filtered and concentrated to dryness. The resulting solid residue was triturated in a 1:1 mixture of hexane:water $(20 \mathrm{~mL})$ and then filtered to give compound $(S)-\mathbf{X}$ as a white solid; yield: $2.69 \mathrm{~g}(85 \%)$; mp: $131-133^{\circ} \mathrm{C}$.

${ }^{1} \mathrm{H}$ NMR (300 MHz, $\left.\mathrm{CDCl}_{3}\right): \delta 8.59(\mathrm{ddd}, J=4.8,1.7,0.9 \mathrm{~Hz}, 1 \mathrm{H}), 7.90(\mathrm{dt}, J=7.8,1.1 \mathrm{~Hz}, 1 \mathrm{H}), 7.84$ (td, $J=7.7,1.7 \mathrm{~Hz}, 1 \mathrm{H}), 7.43(\mathrm{ddd}, J=7.5,4.7,1.4 \mathrm{~Hz}, 1 \mathrm{H}), 7.26-7.16(\mathrm{~m}, 3 \mathrm{H}), 7.15-7.09$ (m, 2H), $5.57(\mathrm{~d}, J=8.9 \mathrm{~Hz}, 1 \mathrm{H}), 4.58(\mathrm{dt}, J=8.8,6.0 \mathrm{~Hz}, 1 \mathrm{H}), 3.98(\mathrm{qq}, J=7.1,3.6 \mathrm{~Hz}, 2 \mathrm{H}), 3.10(\mathrm{~d}, J=6.1$ $\mathrm{Hz}, 2 \mathrm{H}), 1.10(\mathrm{t}, J=7.1 \mathrm{~Hz}, 3 \mathrm{H}) .{ }^{13} \mathrm{C}\left\{{ }^{1} \mathrm{H}\right\} \mathrm{NMR}\left(75 \mathrm{MHz}, \mathrm{CDCl}_{3}\right): \delta 170.9,157.8,149.8,138.0,135.2$, 129.6, 128.5, 127.2, 126.7, 121.7, 61.7, 57.6, 39.8, 14.0. ESI-HRMS: Calcd for $\mathrm{C}_{16} \mathrm{H}_{19} \mathrm{~N}_{2} \mathrm{O}_{4} \mathrm{~S}[\mathrm{M}+\mathrm{H}]^{+}$: 335.1060. Found: 335.1052. $[\alpha] \mathrm{D}^{298}=-9.75\left(\mathrm{c}=1.11, \mathrm{CHCl}_{3}\right)$.

( \pm -Ethyl 3-(4-methoxyphenyl)-2-(pyridine-2-sulfonamido)propanoate $[( \pm)-\mathrm{XI}]$. Following the typical procedure, $( \pm)-\mathrm{II}(694.4 \mathrm{mg}, 3.11 \mathrm{mmol})$ and pyridine $(905.6 \mu \mathrm{L}, 11.20 \mathrm{mmol})$ in THF $(20 \mathrm{~mL})$ 
Revised manuscript. Article published in J. Org. Chem. 2015, 80, 3321-3331 (DOI: $10.1021 / j 0502912 \mathrm{~m}$ )

reacted with a soln of 2-pyridylsulfonyl chloride $(662.9 \mathrm{mg}, 3.73 \mathrm{mmol})$ in THF $(5 \mathrm{~mL})$ to give $( \pm)$-XI as a white solid; yield: $829.4 \mathrm{~g}(73 \%)$; mp: $125-128^{\circ} \mathrm{C}$.

${ }^{1} \mathrm{H}$ NMR (300 MHz, $\left.\mathrm{CDCl}_{3}\right): \delta 8.59(\mathrm{~d}, J=4.6 \mathrm{~Hz}, 1 \mathrm{H}), 7.89(\mathrm{~d}, J=8.0 \mathrm{~Hz}, 1 \mathrm{H}), 7.84$ (td, $J=7.7,1.7$ Hz, 1H), 7.43 (ddd, $J=7.4,4.7,1.3 \mathrm{~Hz}, 1 \mathrm{H}), 7.03(\mathrm{~d}, J=8.6 \mathrm{~Hz}, 2 \mathrm{H}), 6.75(\mathrm{~d}, J=8.6 \mathrm{~Hz}, 2 \mathrm{H}), 5.53(\mathrm{~d}$, $J=8.8 \mathrm{~Hz}, 1 \mathrm{H}), 4.53(\mathrm{dt}, J=8.8,5.9 \mathrm{~Hz}, 1 \mathrm{H}), 3.98(\mathrm{~m}, 2 \mathrm{H}), 3.75(\mathrm{~s}, 3 \mathrm{H}), 3.04(\mathrm{~d}, J=5.9 \mathrm{~Hz}, 2 \mathrm{H}), 1.12$ $(\mathrm{t}, J=7.1 \mathrm{~Hz}, 3 \mathrm{H}) .{ }^{13} \mathrm{C}\left\{{ }^{1} \mathrm{H}\right\} \mathrm{NMR}\left(75 \mathrm{MHz}, \mathrm{CDCl}_{3}\right): \delta 170.9,158.8,157.9,149.8,138.0,130.6,127.1$, 126.7, 121.7, 113.9, 61.6, 57.7, 55.3, 38.9, 14.1. ESI-HRMS: Calcd for $\mathrm{C}_{17} \mathrm{H}_{20} \mathrm{~N}_{2} \mathrm{O}$ S $[\mathrm{M}+\mathrm{H}]^{+}$: 365.1166. Found: 365.1143.

( \pm )-Ethyl 2-(pyridine-2-sulfonamido)-3-(p-tolyl)propanoate $[( \pm)-X I I]$. Following the typical procedure, $( \pm)$-III $(461.0 \mathrm{mg}, 2.22 \mathrm{mmol})$ and pyridine $(647.6 \mu \mathrm{L}, 8.00 \mathrm{mmol})$ in THF $(20 \mathrm{~mL})$ reacted with a soln of 2-pyridylsulfonyl chloride $(474.0 \mathrm{mg}, 2.67 \mathrm{mmol})$ in THF $(5 \mathrm{~mL})$ to give $( \pm)$-XII as a yellow solid. Further purification by column chromatography (hexane-EtOAc, 2:3) provided $( \pm)$-XII as a white powder; yield: $389.3 \mathrm{mg}(50 \%)$; mp: $129-131{ }^{\circ} \mathrm{C}$.

${ }^{1} \mathrm{H}$ NMR (300 MHz, $\left.\mathrm{CDCl}_{3}\right): \delta 8.59(\mathrm{dq}, J=4.7,1.3 \mathrm{~Hz}, 1 \mathrm{H}), 7.89(\mathrm{dt}, J=7.8,1.3 \mathrm{~Hz}, 1 \mathrm{H}), 7.84(\mathrm{td}, J$ = 7.6, 1.7 Hz, 1H), $7.43(\mathrm{ddd}, J=7.3,4.7,1.5 \mathrm{~Hz}, 1 \mathrm{H}), 7.05-6.98(\mathrm{~m}, 4 \mathrm{H}), 5.46(\mathrm{~d}, J=8.9 \mathrm{~Hz}, 1 \mathrm{H})$, $4.56(\mathrm{dt}, J=8.8,5.9 \mathrm{~Hz}, 1 \mathrm{H}), 3.99(\mathrm{qd}, J=7.1,2.2 \mathrm{~Hz}, 2 \mathrm{H}), 3.06(\mathrm{~d}, J=5.9 \mathrm{~Hz}, 2 \mathrm{H}), 2.28(\mathrm{~s}, 3 \mathrm{H}), 1.12$ $(\mathrm{t}, J=7.1 \mathrm{~Hz}, 3 \mathrm{H}) .{ }^{13} \mathrm{C}\left\{{ }^{1} \mathrm{H}\right\} \mathrm{NMR}\left(75 \mathrm{MHz}, \mathrm{CDCl}_{3}\right): \delta 170.9,157.8,149.8,138.0,136.7,131.9,129.4$, 129.2, 126.6, 121.7, 61.6, 57.6, 39.3, 21.1, 14.0. ESI-HRMS: Calcd for $\mathrm{C}_{17} \mathrm{H}_{21} \mathrm{~N}_{2} \mathrm{O} 4 \mathrm{~S}[\mathrm{M}+\mathrm{H}]^{+}$: 349.1217. Found: 349.1212.

( \pm )-Ethyl 3-(4-fluorophenyl)-2-(pyridine-2-sulfonamido)propanoate $[( \pm)-\mathrm{XIII}]$. Following the typical procedure, $( \pm)-\mathbf{I V}(851.0 \mathrm{mg}, 4.03 \mathrm{mmol})$ and pyridine $(1170 \mu \mathrm{L}, 14.50 \mathrm{mmol})$ in THF $(20 \mathrm{~mL})$ reacted with a soln of 2-pyridylsulfonyl chloride $(858.7 \mathrm{mg}, 4.835 \mathrm{mmol})$ in THF (5 mL) to give $( \pm)$ XIII as a white solid. Yield: $1.24 \mathrm{~g}(87 \%)$; mp: $132-134{ }^{\circ} \mathrm{C}$. 
Revised manuscript. Article published in J. Org. Chem. 2015, 80, 3321-3331 (DOI: $10.1021 / j 0502912 \mathrm{~m}$ )

${ }^{1} \mathrm{H}$ NMR (300 MHz, $\left.\mathrm{CDCl}_{3}\right): \delta 8.59(\mathrm{ddd}, J=4.8,1.7,1.0 \mathrm{~Hz}, 1 \mathrm{H}), 7.90(\mathrm{dt}, J=7.8,1.3 \mathrm{~Hz}, 1 \mathrm{H}), 7.85$ (td, $J=7.6,1.7 \mathrm{~Hz}, 1 \mathrm{H}), 7.45(\mathrm{ddd}, J=7.4,4.7,1.4 \mathrm{~Hz}, 1 \mathrm{H}), 7.11(\mathrm{~m}, 2 \mathrm{H}), 6.91(\mathrm{~m}, 2 \mathrm{H}), 5.60(\mathrm{~d}, J=$ $8.7 \mathrm{~Hz}, 1 \mathrm{H}), 4.55(\mathrm{dt}, J=8.7,6.0 \mathrm{~Hz}, 1 \mathrm{H}), 4.00(\mathrm{qd}, J=7.1,3.1 \mathrm{~Hz}, 2 \mathrm{H}), 3.10(\mathrm{dd}, J=13.8,6.0 \mathrm{~Hz}$, 1H), $3.05(\mathrm{dd}, J=13.6,6.1 \mathrm{~Hz}, 1 \mathrm{H}), 1.12(\mathrm{t}, J=7.1 \mathrm{~Hz}, 3 \mathrm{H}) .{ }^{13} \mathrm{C}\left\{{ }^{1} \mathrm{H}\right\}$ NMR $\left(75 \mathrm{MHz}, \mathrm{CDCl}_{3}\right): \delta$ $170.84,162.13(\mathrm{~d}, J=245.5 \mathrm{~Hz}), 157.88,149.91,138.14,131.20\left(\mathrm{~d}, J_{C-F}=8.1 \mathrm{~Hz}\right), 131.05\left(\mathrm{~d}, J_{C-F}=3.3\right.$ $\mathrm{Hz}), 126.81,121.76,115.43\left(\mathrm{~d}, J_{C-F}=21.3 \mathrm{~Hz}\right), 61.86,57.71\left(\mathrm{~d}, J_{C-F}=1.4 \mathrm{~Hz}\right), 39.05,14.12 .{ }^{19} \mathrm{~F}\left\{{ }^{1} \mathrm{H}\right\}$ NMR: $\delta$-115.55 (s). ESI-HRMS: Calcd for $\mathrm{C}_{16} \mathrm{H}_{18} \mathrm{FN}_{2} \mathrm{O}_{4} \mathrm{~S}$ [M+H] $]^{+}: 353.0966$. Found: 353.0991.

( \pm -Ethyl 3-(4-chlorophenyl)-2-(pyridine-2-sulfonamido)propanoate $[( \pm)-X I V]$. Following the typical procedure, $( \pm)-\mathbf{V}(1.12 \mathrm{~g}, 4.93 \mathrm{mmol})$ and pyridine $(718.2 \mu \mathrm{L}, 8.88 \mathrm{mmol})$ in $\mathrm{THF}(20 \mathrm{~mL})$ reacted with a soln of 2-pyridylsulfonyl chloride (1.05 g, $5.92 \mathrm{mmol})$ in THF (5 mL) to give ( \pm )-XIV as a white solid; yield: $1.42 \mathrm{~g}(78 \%)$; mp: $129-131^{\circ} \mathrm{C}$.

${ }^{1} \mathrm{H}$ NMR (300 MHz, CDCl 3$): \delta 8.59(\mathrm{dt}, J=4.8,1.3 \mathrm{~Hz}, 1 \mathrm{H}), 7.89(\mathrm{~d}, J=8.3 \mathrm{~Hz}, 1 \mathrm{H}), 7.85(\mathrm{td}, J=8.0$, $1.5 \mathrm{~Hz}, 1 \mathrm{H}), 7.45$ (ddd, $J=6.9,4.8,1.8 \mathrm{~Hz}, 1 \mathrm{H}), 7.19$ (d, $J=8.4 \mathrm{~Hz}, 2 \mathrm{H}), 7.07(\mathrm{~d}, J=8.4 \mathrm{~Hz}, 2 \mathrm{H}), 5.47$ $(\mathrm{d}, J=8.6 \mathrm{~Hz}, 1 \mathrm{H}), 4.58(\mathrm{dt}, J=8.6,6.0 \mathrm{~Hz}, 1 \mathrm{H}), 4.03(\mathrm{qd}, J=7.1,1.7 \mathrm{~Hz}, 2 \mathrm{H}), 3.12(\mathrm{dd}, J=13.9,5.9$ $\mathrm{Hz}, 1 \mathrm{H}), 3.04(\mathrm{dd}, J=13.9,6.2 \mathrm{~Hz}, 1 \mathrm{H}), 1.15(\mathrm{t}, J=7.1 \mathrm{~Hz}, 3 \mathrm{H}) .{ }^{13} \mathrm{C}\left\{{ }^{1} \mathrm{H}\right\} \mathrm{NMR}\left(75 \mathrm{MHz}, \mathrm{CDCl}_{3}\right): \delta$ $170.7,149.9,138.1,133.8,133.2,131.0,128.7,126.8,121.7,61.9,57.6,39.2,14.1$ [The quaternary $\mathrm{C}$ atom of the pi fragment was not observed, in spite of the use of long accumulation periods]. ESIHRMS: Calcd for $\mathrm{C}_{16} \mathrm{H}_{18} \mathrm{ClN}_{2} \mathrm{O}_{4} \mathrm{~S}[\mathrm{M}+\mathrm{H}]^{+}: 369.0670$. Found: 369.0663 .

( \pm )-Ethyl 3-(4-bromophenyl)-2-(pyridine-2-sulfonamido)propanoate $[( \pm)-\mathrm{XV}]$. Following the typical procedure, $( \pm)$-VI $(1.04 \mathrm{~g}, 3.82 \mathrm{mmol})$ and pyridine $(556.4 \mu \mathrm{L}, 6.88 \mathrm{mmol})$ in $\mathrm{THF}(5 \mathrm{~mL})$ reacted with a soln of 2-pyridylsulfonyl chloride $(814.5 \mathrm{mg}, 4.59 \mathrm{mmol})$ in THF $(20 \mathrm{~mL})$ to give $( \pm)$ $\mathbf{X V}$ as a white solid; yield: $1.217 \mathrm{~g}(77 \%) ; \mathrm{mp}: 126-128^{\circ} \mathrm{C}$.

${ }^{1} \mathrm{H}$ NMR (300 MHz, $\left.\mathrm{CDCl}_{3}\right): \delta 8.58(\mathrm{dt}, J=4.6,1.3 \mathrm{~Hz}, 1 \mathrm{H}), 7.88(\mathrm{~d}, J=7.0 \mathrm{~Hz}, 1 \mathrm{H}) 7.84(\mathrm{td}, J=7.9$, $1.4 \mathrm{~Hz}, 1 \mathrm{H}), 7.45(\mathrm{ddd}, J=6.8,4.8,2.1 \mathrm{~Hz}, 1 \mathrm{H}), 7.33(\mathrm{~d}, J=8.3 \mathrm{~Hz}, 2 \mathrm{H}), 7.01(\mathrm{~d}, J=8.3 \mathrm{~Hz}, 2 \mathrm{H}), 5.59$ 
Revised manuscript. Article published in J. Org. Chem. 2015, 80, 3321-3331 (DOI: $10.1021 / j o 502912 \mathrm{~m}$ )

$(\mathrm{d}, J=8.7 \mathrm{~Hz}, 1 \mathrm{H}), 4.56(\mathrm{dt}, J=8.7,6.1 \mathrm{~Hz}, 1 \mathrm{H}), 4.03(\mathrm{qd}, J=7.1,1.5 \mathrm{~Hz}, 2 \mathrm{H}), 3.09(\mathrm{dd}, J=13.9$, $5.8 \mathrm{~Hz}, 1 \mathrm{H}), 3.01(\mathrm{dd}, J=13.8,6.4 \mathrm{~Hz}, 1 \mathrm{H}), 1.14(\mathrm{t}, J=7.1 \mathrm{~Hz}, 3 \mathrm{H}) .{ }^{13} \mathrm{C}\left\{{ }^{1} \mathrm{H}\right\} \mathrm{NMR}\left(75 \mathrm{MHz}, \mathrm{CDCl}_{3}\right)$ : $\delta 170.7,157.8,149.9,138.1,134.4,131.6,131.3,126.7,121.7,121.3,61.9,57.5,39.2,14.1$. ESIHRMS: Calcd for $\mathrm{C}_{16} \mathrm{H}_{18} \mathrm{BrN}_{2} \mathrm{O}_{4} \mathrm{~S}[\mathrm{M}+\mathrm{H}]^{+}$: 413.0165. Found: 413.0159.

( \pm )-Ethyl 3-(3-fluorophenyl)-2-(pyridine-2-sulfonamido)propanoate $[( \pm)-X V I]$. Following the typical procedure, $( \pm)$-VII $(316.8 \mathrm{mg}, 1.50 \mathrm{mmol})$ and pyridine $(548.8 \mu \mathrm{L}, 6.78 \mathrm{mmol})$ in THF $(20 \mathrm{~mL})$ reacted with a soln of 2-pyridylsulfonyl chloride $(401.7 \mathrm{mg}, 2.26 \mathrm{mmol})$ in THF $(5 \mathrm{~mL})$ to give $( \pm)$-XVI as a white solid; yield: $422.8 \mathrm{mg}(80 \%)$; $\mathrm{mp}: 144-146^{\circ} \mathrm{C}$.

${ }^{1} \mathrm{H}$ NMR $\left(300 \mathrm{MHz}, \mathrm{CDCl}_{3}\right): \delta 8.53(\mathrm{dt}, J=0.7,4.7 \mathrm{~Hz}, 1 \mathrm{H}), 7.85(\mathrm{dd}, J=0.7,7.5 \mathrm{~Hz}, 1 \mathrm{H}), 7.80(\mathrm{dt}, J$ $=1.6,8.9 \mathrm{~Hz}, 1 \mathrm{H}), 7.39(\mathrm{~m}, 1 \mathrm{H}), 7.16(\mathrm{~m}, 1 \mathrm{H}), 6.88-6.74(\mathrm{~m}, 3 \mathrm{H}), 5.27(\mathrm{~d}, J=8.5 \mathrm{~Hz}, 1 \mathrm{H}), 4.52(\mathrm{~m}$, $1 \mathrm{H}), 3.95(\mathrm{dc}, J=5.5,7.0 \mathrm{~Hz}, 2 \mathrm{H}), 3.04(\mathrm{~d}, J=5.8 \mathrm{~Hz}, 2 \mathrm{H}), 1.07(\mathrm{t}, J=7.1 \mathrm{~Hz}, 3 \mathrm{H}) .{ }^{13} \mathrm{C}\left\{{ }^{1} \mathrm{H}\right\} \mathrm{NMR}(75$ $\left.\mathrm{MHz}, \mathrm{CDCl}_{3}\right): \delta 170.6,164.2\left(\mathrm{~d}, J_{\mathrm{C}-\mathrm{F}}=245.9 \mathrm{~Hz}\right), 157.8,149.8,138.0,137.7\left(\mathrm{~d}, J_{\mathrm{C}-\mathrm{F}}=7.1 \mathrm{~Hz}\right), 130.0$ $\left(\mathrm{d}, J_{\mathrm{C}-\mathrm{F}}=8.2 \mathrm{~Hz}\right), 126.7,125.3\left(\mathrm{~d}, J_{\mathrm{C}-\mathrm{F}}=2.7 \mathrm{~Hz}\right), 121.6,116.4\left(\mathrm{~d}, J_{\mathrm{C}-\mathrm{F}}=20.0 \mathrm{~Hz}\right), 114.1\left(\mathrm{~d}, J_{\mathrm{C}-\mathrm{F}}=20.9\right.$ $\mathrm{Hz}), 61.8,57.4,39.5,14.0 .{ }^{19} \mathrm{~F}\left\{{ }^{1} \mathrm{H}\right\}$ NMR (282 $\left.\mathrm{MHz}, \mathrm{CDCl}_{3}\right): \delta$-113.0. ESI-HRMS: Calcd for $\mathrm{C}_{16} \mathrm{H}_{18} \mathrm{FN}_{2} \mathrm{O}_{4} \mathrm{~S}[\mathrm{M}+\mathrm{H}]^{+}:$353.0966. Found: 353.0961.

( \pm )-Ethyl 3-(naphthalen-1-yl)-2-(pyridine-2-sulfonamido)propanoate $[( \pm)-X V I I]$. Following the typical procedure, $( \pm)$-VIII $(364.9 \mathrm{mg}, 1.50 \mathrm{mmol})$ and pyridine $(548.8 \mu \mathrm{L}, 6.78 \mathrm{mmol})$ in THF $(20 \mathrm{~mL})$ reacted with a soln of 2-pyridylsulfonyl chloride $(401.7 \mathrm{mg}, 2.26 \mathrm{mmol})$ in THF $(5 \mathrm{~mL})$ to give $( \pm)$ XVII as a colorless oil; yield: $409.5 \mathrm{mg}(71 \%)$.

${ }^{1} \mathrm{H}$ NMR (300 MHz, $\left.\mathrm{CDCl}_{3}\right): \delta 8.53(\mathrm{~m}, 1 \mathrm{H}), 7.85-7.72(\mathrm{~m}, 5 \mathrm{H}), 7.59(\mathrm{~s}, 1 \mathrm{H}), 7.48-7.49(\mathrm{~m}, 2 \mathrm{H})$, 7.34$7.25(\mathrm{~m}, 3 \mathrm{H}), 4.70(\mathrm{~m}, 1 \mathrm{H}), 4.05(\mathrm{c}, J=7.0 \mathrm{~Hz}, 2 \mathrm{H}), 3.31-3.27(\mathrm{~m}, 2 \mathrm{H}), 1.13(\mathrm{t}, J=7.2 \mathrm{~Hz}, 3 \mathrm{H})$. ${ }^{13} \mathrm{C}\left\{{ }^{1} \mathrm{H}\right\} \mathrm{NMR}\left(75 \mathrm{MHz}, \mathrm{CDCl}_{3}\right): \delta 171.0,157.6,149.7,137.8,133.3,133.0,132.5,128.4,128.1,127.7$, 127.6, 127.5, 126.4, 126.0, 125.8, 121.5, 61.7, 57.8, 39.8, 14.0. ESI-HRMS: Calcd for $\mathrm{C}_{20} \mathrm{H}_{21} \mathrm{~N}_{2} \mathrm{O}_{4} \mathrm{~S}$ $[\mathrm{M}+\mathrm{H}]^{+}:$385.1217. Found: 385.1211. 
Revised manuscript. Article published in J. Org. Chem. 2015, 80, 3321-3331 (DOI: $10.1021 / j 0502912 \mathrm{~m}$ )

( \pm )-Ethyl 3-(naphthalen-2-yl)-2-(pyridine-2-sulfonamido)propanoate $[( \pm)-X V I I I]$. Following the typical procedure, $( \pm)-\mathbf{I X}(364.9 \mathrm{mg}, 1.50 \mathrm{mmol})$ and pyridine $(548.8 \mu \mathrm{L}, 6.78 \mathrm{mmol})$ in THF $(20 \mathrm{~mL})$ reacted with a soln of 2-pyridylsulfonyl chloride $(401.7 \mathrm{mg}, 2.26 \mathrm{mmol})$ in THF $(5 \mathrm{~mL})$ to give $( \pm)$ XVIII as a white solid; yield: $440.0 \mathrm{mg}(77 \%)$; mp: $53-55^{\circ} \mathrm{C}$.

${ }^{1} \mathrm{H}$ NMR $\left(300 \mathrm{MHz}, \mathrm{CDCl}_{3}\right): \delta 8.36(\mathrm{~d}, J=4.6 \mathrm{~Hz}, 1 \mathrm{H}), 7.95(\mathrm{~d}, J=7.2 \mathrm{~Hz}, 1 \mathrm{H}), 7.75-7.64(\mathrm{~m}, 5 \mathrm{H})$, 7.44-7.39 (m, 2H), 7.28-7.20 (m, 2H), $5.42(\mathrm{~d}, J=8.8 \mathrm{~Hz}, 1 \mathrm{H}), 4.61(\mathrm{c}, J=7.0 \mathrm{~Hz}, 1 \mathrm{H}), 3.83(\mathrm{~m}, 2 \mathrm{H})$, $3.46(\mathrm{~d}, J=7.1 \mathrm{~Hz}, 2 \mathrm{H}), 0.90(\mathrm{t}, J=7.0 \mathrm{~Hz}, 3 \mathrm{H}){ }^{13} \mathrm{C}\left\{{ }^{1} \mathrm{H}\right\} \mathrm{NMR}\left(75 \mathrm{MHz}, \mathrm{CDCl}_{3}\right): \delta$ 171.3, 157.7, $149.6,137.7,133.8,131.8,131.6,128.8,128.1,128.0,126.4,126.3,125.7,125.3,123.5,121.4,61.6$, 57.2, 37.2, 13.8. ESI-HRMS: Calcd for $\mathrm{C}_{20} \mathrm{H}_{21} \mathrm{~N}_{2} \mathrm{O}_{4} \mathrm{~S}[\mathrm{M}+\mathrm{H}]^{+}$, 385.1217. Found: 385.1215.

\section{Step 3: $N$-methylation}

Typical procedure: synthesis of $(S)$-ethyl 2-( $N$-methylpyridine-2-sulfonamido)-3phenylpropanoate $[(S)-1]$. To a solution of $(S)-\mathbf{X}(2.69 \mathrm{~g}, 8.05 \mathrm{mmol})$ and $\mathrm{K}_{2} \mathrm{CO}_{3}(2.23 \mathrm{~g}, 16.11 \mathrm{mmol})$ in $\mathrm{MeCN}(20 \mathrm{~mL})$, was added methyl iodide $(1.5 \mathrm{~mL}, 24.16 \mathrm{mmol})$. The reaction mixture was refluxed for $16 \mathrm{~h}$ before it was cooled to room temperature and concentrated to dryness. The residue was purified by flash chromatography ( $n$-hexane-EtOAc 9:1) to give compound $(S)$-1. Furhter purification by trituration in a 1:2 mixture $n$-hexane-Et $2 \mathrm{O}$ gave $(S)$-1 as a white solid; yield: $2.21 \mathrm{~g}(79 \%)$; mp: $119-$ $121^{\circ} \mathrm{C}$

${ }^{1} \mathrm{H}$ NMR (300 MHz, $\left.\mathrm{CDCl}_{3}\right): \delta 8.61(\mathrm{dt}, J=4.7,1.6 \mathrm{~Hz}, 1 \mathrm{H}), 7.85-7.75(\mathrm{~m}, 2 \mathrm{H}), 7.42(\mathrm{ddd}, J=6.1$, 4.7, 2.6 Hz, 1H), $7.25-7.15(\mathrm{~m}, 5 \mathrm{H}), 4.96(\mathrm{dd}, J=8.3,7.3 \mathrm{~Hz}, 1 \mathrm{H}), 3.95(\mathrm{q}, J=7.1 \mathrm{~Hz}, 2 \mathrm{H}), 3.24(\mathrm{dd}$, $J=14.0,7.3 \mathrm{~Hz}, 1 \mathrm{H}), 3.07(\mathrm{~s}, 3 \mathrm{H}), 2.96(\mathrm{dd}, J=14.0,8.3 \mathrm{~Hz}, 1 \mathrm{H}), 1.05(\mathrm{t}, J=7.1 \mathrm{~Hz}, 3 \mathrm{H}) .{ }^{13} \mathrm{C}\left\{{ }^{1} \mathrm{H}\right\}$ NMR (75 MHz, $\left.\mathrm{CDCl}_{3}\right): \delta 170.1,157.5,149.8,137.7,136.3,129.1,128.6,126.9,126.4,122.1,61.2$, 61.0, 35.9, 31.3, 14.0. ESI-HRMS: Calcd for $\mathrm{C}_{17} \mathrm{H}_{21} \mathrm{~N}_{2} \mathrm{O}_{4} \mathrm{~S}[\mathrm{M}+\mathrm{H}]^{+}: 349.1217$. Found: 349.1190 . [ $\left.\alpha\right]_{\mathrm{D}}^{298}$ $=-19.12\left(c 1.00, \mathrm{CHCl}_{3}\right)$. 
Revised manuscript. Article published in J. Org. Chem. 2015, 80, 3321-3331 (DOI: 10.1021/jo502912m)

( \pm )-Ethyl 3-(4-methoxyphenyl)-2-(N-methylpyridine-2-sulfonamido)propanoate $\quad[( \pm)$-XIX]. Following the typical procedure, $( \pm)$-XI $(829.4 \mathrm{mg}, 2.28 \mathrm{mmol})$ reacted with $\mathrm{MeI}(425.1 \mu \mathrm{L}, 6.828$ mmol) in the presence of $\mathrm{K}_{2} \mathrm{CO}_{3}(629.1 \mathrm{mg}, 4.55 \mathrm{mmol})$ in $\mathrm{MeCN}$ (10 mL) to give, after column chromatography (n-hexane-EtOAc 8:2), $( \pm)$-XIX as a white solid; yield: $669.9 \mathrm{mg}$ (78\%); mp: 115 $117^{\circ} \mathrm{C}$.

${ }^{1} \mathrm{H}$ NMR $\left(300 \mathrm{MHz}, \mathrm{CDCl}_{3}\right): \delta 8.56(\mathrm{~d}, J=4.7 \mathrm{~Hz}, 1 \mathrm{H}), 7.84-7.67(\mathrm{~m}, 2 \mathrm{H}), 7.39(\mathrm{t}, J=5.7 \mathrm{~Hz}, 1 \mathrm{H})$, $7.05(\mathrm{~d}, J=8.3 \mathrm{~Hz}, 2 \mathrm{H}), 6.71(\mathrm{~d}, J=8.2 \mathrm{~Hz}, 2 \mathrm{H}), 4.87(\mathrm{t}, J=7.8 \mathrm{~Hz}, 1 \mathrm{H}), 3.92(\mathrm{q}, J=7.1 \mathrm{~Hz}, 2 \mathrm{H}), 3.71$ (s, 3H), $3.14(\mathrm{dd}, J=14.2,7.1 \mathrm{~Hz}, 1 \mathrm{H}), 3.03(\mathrm{~s}, 3 \mathrm{H}), 2.85(\mathrm{dd}, J=14.1,8.5 \mathrm{~Hz}, 1 \mathrm{H}), 1.02(\mathrm{t}, J=7.1 \mathrm{~Hz}$, $3 \mathrm{H}) .{ }^{13} \mathrm{C}\left\{{ }^{1} \mathrm{H}\right\} \mathrm{NMR}\left(75 \mathrm{MHz}, \mathrm{CDCl}_{3}\right): \delta 170.0,158.3,157.3,149.7,137.6,129.9,128.1,126.3,121.9$, 113.8, 61.0, 60.9, 55.0, 34.8, 31.1, 13.9. ESI-HRMS: Calcd for $\mathrm{C}_{18} \mathrm{H}_{23} \mathrm{~N}_{2} \mathrm{O}_{5} \mathrm{~S}[\mathrm{M}+\mathrm{H}]^{+}: 379.1328$. Found: 379.1322.

( \pm -Ethyl 2-(N-methylpyridine-2-sulfonamido)-3-(p-tolyl)propanoate $[( \pm)-\mathrm{XX}]$. Following the typical procedure, $( \pm)$-XII $(389.3 \mathrm{mg}, 1.12 \mathrm{mmol})$ reacted with MeI $(208.6 \mu \mathrm{L}, 3.35 \mathrm{mmol})$ in the presence of $\mathrm{K}_{2} \mathrm{CO}_{3}(308.8 \mathrm{mg}, 2.23 \mathrm{mmol})$ in $\mathrm{MeCN}(10 \mathrm{~mL})$ to give, after column chromatography $(n$ hexane-EtOAc 8:2), $( \pm)-\mathbf{X X}$ as a white solid; yield: $387.3 \mathrm{mg}(96 \%)$; $\mathrm{mp}: 121-123{ }^{\circ} \mathrm{C}$.

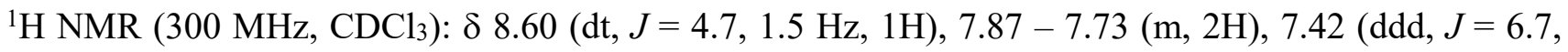
4.7, $2.3 \mathrm{~Hz}, 1 \mathrm{H}), 7.09-7.00(\mathrm{~m}, 4 \mathrm{H}), 4.93(\mathrm{dd}, J=8.6,7.0 \mathrm{~Hz}, 1 \mathrm{H}), 3.96(\mathrm{q}, J=7.1 \mathrm{~Hz}, 2 \mathrm{H}), 3.20(\mathrm{dd}$, $J=14.1,7.0 \mathrm{~Hz}, 1 \mathrm{H}), 3.07(\mathrm{~s}, 3 \mathrm{H}), 2.90(\mathrm{dd}, J=14.0,8.5 \mathrm{~Hz}, 1 \mathrm{H}), 2.29(\mathrm{~s}, 3 \mathrm{H}), 1.07(\mathrm{t}, J=7.1 \mathrm{~Hz}$ $3 \mathrm{H}) .{ }^{13} \mathrm{C}\left\{{ }^{1} \mathrm{H}\right\} \mathrm{NMR}\left(75 \mathrm{MHz}, \mathrm{CDCl}_{3}\right): \delta 170.2,157.6,149.8,137.7,136.4,133.2,129.3,129.0,126.3$, 122.1, 61.2, 61.0, 35.5, 31.3, 21.2, 14.0. ESI-HRMS: Calcd for $\mathrm{C}_{18} \mathrm{H}_{23} \mathrm{~N}_{2} \mathrm{O}_{4} \mathrm{~S}[\mathrm{M}+\mathrm{H}]^{+}: 363.1373$. Found: 363.1388.

( \pm )-Ethyl 3-(4-fluorophenyl)-2-(N-methylpyridine-2-sulfonamido)propanoate $[( \pm)-X X I]$. Following the typical procedure, $( \pm)$-XIII $(1.24 \mathrm{~g}, 3.51 \mathrm{mmol})$ reacted with $\mathrm{MeI}(656.3 \mu \mathrm{L}, 10.54 \mathrm{mmol})$ in the 
Revised manuscript. Article published in J. Org. Chem. 2015, 80, 3321-3331 (DOI: $10.1021 / j 0502912 \mathrm{~m}$ )

presence of $\mathrm{K}_{2} \mathrm{CO}_{3}(971.4 \mathrm{mg}, 7.03 \mathrm{mmol})$ in $\mathrm{MeCN}(10 \mathrm{~mL})$ to give, after column chromatography $(n$ hexane-EtOAc 8:2), ( \pm )-XXI as a white solid; yield: $1.027 \mathrm{~g}(80 \%)$; mp: $121-123{ }^{\circ} \mathrm{C}$.

${ }^{1} \mathrm{H}$ NMR (300 MHz, $\left.\mathrm{CDCl}_{3}\right): \delta 8.53(\mathrm{ddd}, J=4.8,1.7,0.9 \mathrm{~Hz}, 1 \mathrm{H}), 7.76(\mathrm{td}, J=7.7,1.7 \mathrm{~Hz}, 1 \mathrm{H}), 7.71$ (ddd, $J=7.9,1.5,0.9 \mathrm{~Hz}, 1 \mathrm{H}), 7.37(\mathrm{ddd}, J=7.4,4.7,1.5 \mathrm{~Hz}, 1 \mathrm{H}), 7.14-7.04(\mathrm{~m}, 2 \mathrm{H}), 6.83(\mathrm{t}, J=8.7$ $\mathrm{Hz}, 2 \mathrm{H}), 4.85(\mathrm{dd}, J=8.7,6.9 \mathrm{~Hz}, 1 \mathrm{H}), 3.88(\mathrm{q}, J=7.1 \mathrm{~Hz}, 2 \mathrm{H}), 3.14(\mathrm{dd}, J=14.2,6.9 \mathrm{~Hz}, 1 \mathrm{H}), 2.99$ (s, 3H), $2.86(\mathrm{dd}, J=14.2,8.7 \mathrm{~Hz}, 1 \mathrm{H}), 0.97(\mathrm{t}, J=7.1 \mathrm{~Hz}, 3 \mathrm{H}) \cdot{ }^{13} \mathrm{C}\left\{{ }^{1} \mathrm{H}\right\} \mathrm{NMR}\left(75 \mathrm{MHz}, \mathrm{CDCl}_{3}\right): \delta$ $169.6,161.5\left(\mathrm{~d}, J_{\mathrm{C}-\mathrm{F}}=244.8 \mathrm{~Hz}\right), 157.1,149.6,137.6,131.9\left(\mathrm{~d}, J_{\mathrm{C}-\mathrm{F}}=0.7 \mathrm{~Hz}\right), 130.4\left(\mathrm{~d}, J_{\mathrm{C}-\mathrm{F}}=8.0 \mathrm{~Hz}\right)$, $126.3,121.8,115.1\left(\mathrm{~d}, J_{\mathrm{C}-\mathrm{F}}=21.3 \mathrm{~Hz}\right), 61.0,60.8\left(\mathrm{~d}, J_{\mathrm{C}-\mathrm{F}}=1.3 \mathrm{~Hz}\right), 34.7,31.00,13.7 .{ }^{19} \mathrm{~F}\left\{{ }^{1} \mathrm{H}\right\} \mathrm{NMR}: \delta$ -115.99 (s). ESI-HRMS: Calcd for $\mathrm{C}_{17} \mathrm{H}_{20} \mathrm{FN}_{2} \mathrm{O}_{4} \mathrm{~S}[\mathrm{M}+\mathrm{H}]^{+}$: 367.1122 . Found: 367.1113.

\section{( \pm -Ethyl 3-(4-chlorophenyl)-2-(N-methylpyridine-2-sulfonamido)propanoate $\quad[( \pm)-X X I I]$.}

Following the typical procedure, $( \pm)$-XIV $(1422 \mathrm{mg}, 3.85 \mathrm{mmol})$ reacted with $\mathrm{MeI}(719.9 \mu \mathrm{L}, 11.56$ mmol) in the presence of $\mathrm{K}_{2} \mathrm{CO}_{3}(1.07 \mathrm{~g}, 7.71 \mathrm{mmol})$ in $\mathrm{MeCN}(10 \mathrm{~mL})$ to give, after column chromatography (n-hexane-EtOAc 8:2), ( $( \pm)$-XXII as a white solid; yield: $145 \mathrm{mg}$ (98\%); mp: 122 - 124 ${ }^{\circ} \mathrm{C}$.

${ }^{1} \mathrm{H}$ NMR $\left(300 \mathrm{MHz}, \mathrm{CDCl}_{3}\right): \delta 8.58(\mathrm{ddd}, J=4.7,1.7,0.9 \mathrm{~Hz}, 1 \mathrm{H}), 7.81$ (ddd, $\left.J=7.8,7.3,1.7 \mathrm{~Hz}, 1 \mathrm{H}\right)$, 7.75 (ddd, $J=7.9,1.5,0.9 \mathrm{~Hz}, 1 \mathrm{H}), 7.43$ (ddd, $J=7.3,4.7,1.5 \mathrm{~Hz}, 1 \mathrm{H}), 7.19-7.07$ (m, 4H), 4.92 (dd, $J$ $=8.8,6.8 \mathrm{~Hz}, 1 \mathrm{H}), 3.98(\mathrm{q}, J=7.1 \mathrm{~Hz}, 2 \mathrm{H}), 3.21(\mathrm{dd}, J=14.2,6.8 \mathrm{~Hz}, 1 \mathrm{H}), 3.05(\mathrm{~s}, 3 \mathrm{H}), 2.92(\mathrm{dd}, J=$ 14.2, 8.8 Hz, 1H), $1.08(\mathrm{t}, J=7.1 \mathrm{~Hz}, 3 \mathrm{H}) .{ }^{13} \mathrm{C}\left\{{ }^{1} \mathrm{H}\right\} \mathrm{NMR}\left(75 \mathrm{MHz}, \mathrm{CDCl}_{3}\right): \delta 169.9,157.5,149.8$, 137.7, 134.9, 132.8, 130.5, 128.7, 126.4, 122.0, 61.4, 60.9, 35.1, 31.3, 14.0. ESI-HRMS: Calcd for $\mathrm{C}_{17} \mathrm{H}_{20} \mathrm{ClN}_{2} \mathrm{O}_{4} \mathrm{~S}[\mathrm{M}+\mathrm{H}]^{+}: 383.0832$. Found: 383.0827.

\section{( \pm -Ethyl 3-(4-bromophenyl)-2-(N-methylpyridine-2-sulfonamido)propanoate $[( \pm)-\mathrm{XXIII}]$}

Following the typical procedure, $( \pm)-\mathbf{X V}(1.22 \mathrm{~g}, 2.94 \mathrm{mmol})$ reacted with $\mathrm{MeI}(549.8 \mu \mathrm{L}, 8.83$ mmol) in the presence of $\mathrm{K}_{2} \mathrm{CO}_{3}(813.7 \mathrm{mg}, 5.89 \mathrm{mmol})$ in $\mathrm{MeCN}(10 \mathrm{~mL})$ to give, after column 
Revised manuscript. Article published in J. Org. Chem. 2015, 80, 3321-3331 (DOI: $10.1021 / j 0502912 \mathrm{~m}$ )

chromatography (n-hexane-EtOAc 8:2), ( $( \pm)$-XXIII as a white solid; yield: 1.19 g (95\%); mp: $118-120$ ${ }^{\circ} \mathrm{C}$.

${ }^{1} \mathrm{H}$ NMR (300 MHz, $\left.\mathrm{CDCl}_{3}\right): \delta 8.57$ (ddd, $\left.J=4.7,1.8,0.9 \mathrm{~Hz}, 1 \mathrm{H}\right), 7.80$ (ddd, $\left.J=7.9,7.4,1.7 \mathrm{~Hz}, 1 \mathrm{H}\right)$, $7.73(\mathrm{ddd}, J=7.9,1.4,0.9 \mathrm{~Hz}, 1 \mathrm{H}), 7.43$ (ddd, $J=7.4,4.7,1.4 \mathrm{~Hz}, 1 \mathrm{H}), 7.33-7.27$ (m, 2H), $7.07-$ $6.99(\mathrm{~m}, 2 \mathrm{H}), 4.91(\mathrm{dd}, J=8.9,6.7 \mathrm{~Hz}, 1 \mathrm{H}), 3.98(\mathrm{q}, J=7.1 \mathrm{~Hz}, 2 \mathrm{H}), 3.18(\mathrm{dd}, J=14.2,6.7 \mathrm{~Hz}, 1 \mathrm{H})$, $3.04(\mathrm{~s}, 3 \mathrm{H}), 2.89(\mathrm{dd}, J=14.2,8.9 \mathrm{~Hz}, 1 \mathrm{H}), 1.07(\mathrm{t}, J=7.1 \mathrm{~Hz}, 3 \mathrm{H}) .{ }^{13} \mathrm{C}\left\{{ }^{1} \mathrm{H}\right\} \mathrm{NMR}\left(75 \mathrm{MHz}, \mathrm{CDCl}_{3}\right)$ : $\delta 169.8,157.4,149.8,137.7,135.4,131.6,130.8,126.4,121.9,120.8,61.4,60.8,35.2,31.2,14.0$. ESIHRMS: Calcd for $\mathrm{C}_{17} \mathrm{H}_{20} \mathrm{BrN}_{2} \mathrm{O}_{4} \mathrm{~S}[\mathrm{M}+\mathrm{H}]^{+}$: 427.0322. Found: 427.0314.

( \pm )-Ethyl 3-(3-fluorophenyl)-2-(N-methylpyridine-2-sulfonamido)propanoate $\quad[( \pm)-X X I V]$. Following the typical procedure, $( \pm)$-XVI $(352.4 \mathrm{mg}, 1.00 \mathrm{mmol})$ reacted with $\mathrm{MeI}(186.8 \mu \mathrm{L}, 3.00$ mmol) in the presence of $\mathrm{K}_{2} \mathrm{CO}_{3}(276.3 \mathrm{mg}, 2.00 \mathrm{mmol})$ in $\mathrm{MeCN}(10 \mathrm{~mL})$ to give, after column chromatography (n-hexane-EtOAc 8:2), ( \pm -XXIV as a colorless oil; yield: $311.4 \mathrm{mg}(85 \%)$.

${ }^{1} \mathrm{H}$ NMR (300 MHz, $\left.\mathrm{CDCl}_{3}\right): \delta 8.66(\mathrm{~d}, J=4.7 \mathrm{~Hz}, 1 \mathrm{H}), 7.86(\mathrm{~m}, 2 \mathrm{H}), 7.48(\mathrm{~m}, 1 \mathrm{H}), 7.24(\mathrm{~m}, 1 \mathrm{H}), 7.03$ $(\mathrm{d}, J=7.7 \mathrm{~Hz}, 1 \mathrm{H}), 6.93(\mathrm{~m}, 2 \mathrm{H}), 4.99(\mathrm{t}, J=8.0 \mathrm{~Hz}, 1 \mathrm{H}), 4.01(\mathrm{c}, J=7.1 \mathrm{~Hz}, 2 \mathrm{H}), 3.27(\mathrm{dd}, J=7.2$, $14.0 \mathrm{~Hz}, 1 \mathrm{H}), 3.10(\mathrm{~s}, 3 \mathrm{H}), 3.00(\mathrm{dd}, J=8.3,14.0 \mathrm{~Hz}, 1 \mathrm{H}), 1.12(\mathrm{t}, J=7.1 \mathrm{~Hz}, 3 \mathrm{H}) .{ }^{13} \mathrm{C}\left\{{ }^{1} \mathrm{H}\right\} \mathrm{NMR}(75$ $\left.\mathrm{MHz}, \mathrm{CDCl}_{3}\right): \delta 169.8,162.7\left(\mathrm{~d}, J_{\mathrm{C}-\mathrm{F}}=46.0 \mathrm{~Hz}\right), 157.3,149.8,138.9\left(\mathrm{~d}, J_{\mathrm{C}-\mathrm{F}}=7.1 \mathrm{~Hz}\right), 137.8,130.0(\mathrm{~d}$, $\left.J_{\mathrm{C}-\mathrm{F}}=9.2 \mathrm{~Hz}\right), 126.5,124.8\left(\mathrm{~d}, J_{\mathrm{C}-\mathrm{F}}=3.0 \mathrm{~Hz}\right), 121.9,115.9\left(\mathrm{~d}, J_{\mathrm{C}-\mathrm{F}}=21.4 \mathrm{~Hz}\right), 113.8\left(\mathrm{~d}, J_{\mathrm{C}-\mathrm{F}}=20.9 \mathrm{~Hz}\right)$, 61.2, 60.8, 35.5, 31.2, 13.9. ${ }^{19} \mathrm{~F}\left\{{ }^{1} \mathrm{H}\right\}$ NMR (282 MHz, $\left.\mathrm{CDCl}_{3}\right): \delta$-113.0. ESI-HRMS: Calcd for $\mathrm{C}_{17} \mathrm{H}_{20} \mathrm{FN}_{2} \mathrm{O}_{4} \mathrm{~S}[\mathrm{M}+\mathrm{H}]^{+}: 367.1122$. Found: 367.1128 .

( \pm -Ethyl 2-( $N$-methylpyridine-2-sulfonamido)-3-(naphthalen-1-yl)propanoate $\quad[( \pm)-X X V]$. Following the typical procedure, $( \pm)$-XVII $(384.5 \mathrm{mg}, 1.00 \mathrm{mmol})$ reacted with $\mathrm{MeI}(186.8 \mu \mathrm{L}, 3.00$ mmol) in the presence of $\mathrm{K}_{2} \mathrm{CO}_{3}(276.3 \mathrm{mg}, 2.00 \mathrm{mmol})$ in $\mathrm{MeCN}(10 \mathrm{~mL})$ to give, after column chromatography ( $n$-hexane-EtOAc 8:2), ( $($ )-XXV as a colorless oil; yield: $354.6 \mathrm{mg}(89 \%)$. 
Revised manuscript. Article published in J. Org. Chem. 2015, 80, 3321-3331 (DOI: $10.1021 / j 0502912 \mathrm{~m}$ )

${ }^{1} \mathrm{H}$ NMR (300 MHz, $\left.\mathrm{CDCl}_{3}\right): \delta 8.46(\mathrm{~d}, J=4.3 \mathrm{~Hz}, 1 \mathrm{H}), 7.81(\mathrm{~m}, 1 \mathrm{H}), 7.73(\mathrm{~m}, 2 \mathrm{H}), 7.65(\mathrm{~d}, J=7.9 \mathrm{~Hz}$, 1H), 7.59-7.51 (m, 2H), 7.50-7.46 (m, 2H), $7.35(\mathrm{dd}, J=1.1,8.6 \mathrm{~Hz}, 1 \mathrm{H}), 7.20(\mathrm{~m}, 1 \mathrm{H}), 5.12(\mathrm{dd}, J=$ $6.8,8.9 \mathrm{~Hz}, 1 \mathrm{H}), 4.05(\mathrm{c}, J=7.2 \mathrm{~Hz}, 2 \mathrm{H}), 3.44(\mathrm{dd}, J=6.7,14.0 \mathrm{~Hz}, 1 \mathrm{H}), 3.18(\mathrm{~s}, 3 \mathrm{H}), 3.13(\mathrm{dd}, J=$ 9.8, $14.0 \mathrm{~Hz}, 1 \mathrm{H}), 1.13(\mathrm{t}, J=7.2 \mathrm{~Hz}, 3 \mathrm{H}) .{ }^{13} \mathrm{C}\left\{{ }^{1} \mathrm{H}\right\} \mathrm{NMR}\left(75 \mathrm{MHz}, \mathrm{CDCl}_{3}\right): \delta 170.0,157.3,149.5$, $137.3,133.9,133.3,132.4,128.2,127.8,127.6,127.1,126.0,125.7,121.5,61.3,61.1,35.9,31.3,14.0$. ESI-HRMS: Calcd for $\mathrm{C}_{21} \mathrm{H}_{23} \mathrm{~N}_{2} \mathrm{O}_{4} \mathrm{~S}[\mathrm{M}+\mathrm{H}]^{+}:$399.1379. Found: 399.1372.

$( \pm)$-Ethyl 2-(N-methylpyridine-2-sulfonamido)-3-(naphthalen-2-yl)propanoate $\quad[( \pm)-X X V I]$. Following the typical procedure, $( \pm)$-XVIII $(384.5 \mathrm{mg}, 1.00 \mathrm{mmol})$ reacted with $\mathrm{MeI}(186.8 \mu \mathrm{L}, 3.00$ mmol) in the presence of $\mathrm{K}_{2} \mathrm{CO}_{3}(276.3 \mathrm{mg}, 2.00 \mathrm{mmol})$ in $\mathrm{MeCN}$ (10 mL) to give, after column chromatography ( $n$-hexane-EtOAc 8:2), ( \pm -XXVI as a colorless oil; yield: $318.8 \mathrm{mg}(80 \%)$.

${ }^{1} \mathrm{H}$ NMR $\left(300 \mathrm{MHz}, \mathrm{CDCl}_{3}\right): \delta 8.58(\mathrm{~d}, J=4.5 \mathrm{~Hz}, 1 \mathrm{H}), 8.13(\mathrm{~d}, J=8.2 \mathrm{~Hz}, 1 \mathrm{H}), 7.86(\mathrm{~d}, J=7.8 \mathrm{~Hz}$, 1H), 7.74-7.69 (m, 3H), 7.61-7.50 (m, 2H), $7.39(\mathrm{~m}, 1 \mathrm{H}), 7.32-7.28(\mathrm{~m}, 2 \mathrm{H}), 5.17(\mathrm{t}, J=7.3 \mathrm{~Hz}, 1 \mathrm{H})$, $3.98(\mathrm{c}, J=7.0 \mathrm{~Hz}, 2 \mathrm{H}), 3.74(\mathrm{dd}, J=8.0,14.0 \mathrm{~Hz}, 1 \mathrm{H}), 3.50(\mathrm{dd}, J=7.3,14.0 \mathrm{~Hz}, 2 \mathrm{H}), 3.26(\mathrm{~s}, 3 \mathrm{H})$, $1.03(\mathrm{t}, J=7.0 \mathrm{~Hz}, 3 \mathrm{H}) .{ }^{13} \mathrm{C}\left\{{ }^{1} \mathrm{H}\right\} \mathrm{NMR}\left(75 \mathrm{MHz}, \mathrm{CDCl}_{3}\right): \delta 170.2,157.3,149.6,137.6,133.8,132.2$, $131.8,128.9,127.9,127.7,126.4,126.3,125.7,125.3,123.4,121.8,61.1,60.0,33.4,31.2$, 13.9. ESIHRMS: Calcd for $\mathrm{C}_{21} \mathrm{H}_{23} \mathrm{~N}_{2} \mathrm{O} 4 \mathrm{~S}[\mathrm{M}+\mathrm{H}]^{+}:$399.1379. Found: 399.1375 .

\section{$N$-((1R,2S)-1-Methoxy-1-phenylpropan-2-yl)- $N$-methylpyridine-2-sulfonamide [(-)-20]}

Potassium hydride (1.04 g of a $35 \%$ suspension in oil, $364 \mathrm{mg}, 9.1 \mathrm{mmol}$ ) was weighted into a 100 $\mathrm{ml} 3$ neck round bottom flask. The $\mathrm{KH}$ was washed with hexane $(3 \times 4 \mathrm{~mL})$ to remove the mineral oil and THF $(7 \mathrm{~mL})$ was added to the dry $\mathrm{KH}$ powder. To the resulting suspension, cooled to $0{ }^{\circ} \mathrm{C}$, was added drop-wise (over $10 \mathrm{~min}$ ) a soln of L-norpseudoephedrine (1.37 g, $9.1 \mathrm{mmol})$ in THF (15 mL). The reaction was stirred at room temperature for $10 \mathrm{~min}$ before $\mathrm{MeI}(0.567 \mathrm{~mL}, 9.1 \mathrm{mmol})$ was added. The mixture was stirred for $1.5 \mathrm{~h}$ before a $2 \mathrm{~N}$ soln of sodium thiosulfate $(25 \mathrm{~mL})$ was added. The mixture was extracted with diethyl ether $(3 \times 50 \mathrm{~mL})$ and the combined organic phase was dried 
Revised manuscript. Article published in J. Org. Chem. 2015, 80, 3321-3331 (DOI: 10.1021/jo502912m)

$\left(\mathrm{MgSO}_{4}\right)$ and concentrated to dryness. The resulting methyl ether intermediate $[(1 R, 2 S)$-XXVII] was of sufficient purity for use in the next step.

To a solution of crude $(1 R, 2 S)$-XXVII $(9.1 \mathrm{mmol})$ and pyridine $(1.08 \mathrm{~mL}, 13.65 \mathrm{mmol})$ in dry THF $(20 \mathrm{~mL})$, cooled to $-5{ }^{\circ} \mathrm{C}$ and under Ar atmosphere, was added drop-wise a solution of 2-pyridylsulfonyl chloride $(2.4 \mathrm{~g}, 13.65 \mathrm{mmol})$ in dry THF $(5 \mathrm{~mL})$. The mixture was stirred at room temperature for $18 \mathrm{~h}$ and then it was filtered through a small pad of silica gel and the filtrate concentrated to dryness. The resulting solid residue was triturated in a 1:1 mixture of n-hexane-water $(20 \mathrm{~mL})$ and filtered, to give $(1 R, 2 S)$-XXVIII as a white solid that was used in the next step without further purification.

To a solution of $(1 R, 2 S)$-XXVIII $(9.1 \mathrm{mmol})$ and $\mathrm{K}_{2} \mathrm{CO}_{3}(2.51 \mathrm{~g}, 18.2 \mathrm{mmol})$ in $\mathrm{MeCN}(20 \mathrm{~mL})$ at room temperature was added methyl iodide $(1.0 \mathrm{~mL}, 16.1 \mathrm{mmol})$. The reaction mixture was refluxed for $16 \mathrm{~h}$ before it was concentrated to dryness. The residue was purified by column chromatography ( $n$ hexane-EtOAc 8:2) to afford the desired compound (1R,2S)-20 as a colorless oil; yield: $2.011 \mathrm{~g}(69 \%)$.

${ }^{1} \mathrm{H}$ NMR $\left(300 \mathrm{MHz}, \mathrm{CDCl}_{3}\right): \delta 8.45(\mathrm{~d}, J=4.8 \mathrm{~Hz}, 1 \mathrm{H}), 7.83-7.51(\mathrm{~m}, 2 \mathrm{H}), 7.23(\mathrm{ddd}, J=7.4,4.7,1.4$ $\mathrm{Hz}, 1 \mathrm{H}), 7.18-6.88(\mathrm{~m}, 5 \mathrm{H}), 4.29(\mathrm{~d}, J=3.5 \mathrm{~Hz}, 1 \mathrm{H}), 4.00(\mathrm{dd}, J=7.0,3.4 \mathrm{~Hz}, 1 \mathrm{H}), 3.05(\mathrm{~s}, 3 \mathrm{H}), 2.83$ $(\mathrm{s}, 3 \mathrm{H}), 0.79(\mathrm{~d}, J=7.0 \mathrm{~Hz}, 3 \mathrm{H}) .{ }^{13} \mathrm{C}\left\{{ }^{1} \mathrm{H}\right\} \mathrm{NMR}\left(75 \mathrm{MHz}, \mathrm{CDCl}_{3}\right): \delta 155.6,147.6,136.8,135.4,125.9$, 125.2, 124.3, 123.9, 120.0, 85.3, 56.1, 54.8, 28.6, 8.1. ESI-HRMS: Calcd for $\mathrm{C}_{16} \mathrm{H}_{21} \mathrm{~N}_{2} \mathrm{O}_{3} \mathrm{~S}(\mathrm{M}+\mathrm{H})^{+}$: 321,1267. Found: $321,1261 .[\alpha]_{\mathrm{D}}{ }^{298}=-68.23\left(c 0.68, \mathrm{CHCl}_{3}\right)$.

Typical procedure for the $\mathrm{C}-\mathrm{H}$ activation reaction: Synthesis of $(S, E)-B u t y l$ 3-(2-(3-ethoxy-2-(Nmethylpyridine-2-sulfonamido)-3-oxopropyl) phenyl)acrylate $[(\boldsymbol{S})-2]$ A screw-capped test tube was charged with $N$-(2-pyridyl)sulfonyl phenylalanine ethyl ester derivative $(S)$-1 (52.3 mg, $0.15 \mathrm{mmol}), 1$ fluoro-2,4,6-trimethylpyridinium triflate $(130.2 \mathrm{mg}, 0.45 \mathrm{mmol})$ and $\mathrm{Pd}(\mathrm{OAc})_{2}(3.4 \mathrm{mg}, 0.015 \mathrm{mmol})$. The tube was sealed with a rubber septum and then evacuated and backfilled with nitrogen $(3 \mathrm{x})$ before acetic acid $(1 \mathrm{~mL})$ and butyl acrylate $(43.0 \mu \mathrm{L}, 0.30 \mathrm{mmol})$ were successively added. The septum was replaced with a Teflon-coated screw cap and the resulting solution was heated and vigorously stirred at 
Revised manuscript. Article published in J. Org. Chem. 2015, 80, 3321-3331 (DOI: $10.1021 / j 0502912 \mathrm{~m}$ )

$110{ }^{\circ} \mathrm{C}$ for 6 hours. Then, it was cooled to room temperature and diluted with $\mathrm{CH}_{2} \mathrm{Cl}_{2}(5 \mathrm{~mL})$. The resulting suspension was filtered through a plug of Celite (washed with $\mathrm{CH}_{2} \mathrm{Cl}_{2}$ ). The clear solution was washed with sat. aq soln of $\mathrm{NaHCO}_{3}(2 \times 10 \mathrm{~mL})$, dried with anhydrous $\mathrm{MgSO}_{4}$ and then concentrated to dryness. The residue was then purified by column chromatography ( $n$-hexane-EtOAc 85:15) to afford (S)-2 as a yellow oil; yield: $59.8 \mathrm{mg}(84 \%)$.

$[\alpha]_{\mathrm{D}^{20}}=-13.7\left(c 0.89, \mathrm{CHCl}_{3}\right) ;{ }^{1} \mathrm{H} \mathrm{NMR}\left(300 \mathrm{MHz}, \mathrm{CDCl}_{3}\right): \delta 8.57(\mathrm{dd}, J=0.6,7.5 \mathrm{~Hz}, 1 \mathrm{H}), 7.98(\mathrm{~d}$, $J=15.0 \mathrm{~Hz}, 1 \mathrm{H}), 7.75-7.70(\mathrm{~m}, 2 \mathrm{H}), 7.55-7.40(\mathrm{~m}, 1 \mathrm{H}), 7.40-7.35(\mathrm{~m}, 2 \mathrm{H}), 7.30-7.10(\mathrm{~m}, 2 \mathrm{H}), 6.73(\mathrm{~d}$, $J=15.0 \mathrm{~Hz}, 1 \mathrm{H}), 4.80(\mathrm{t}, J=8.0 \mathrm{~Hz}, 1 \mathrm{H}), 3.95-3.80(\mathrm{~m}, 2 \mathrm{H}), 3.33(\mathrm{dd}, J=7.3 \mathrm{~Hz}, 1 \mathrm{H}), 3.10(\mathrm{dd}, J=$ $7.3 \mathrm{~Hz}, 1 \mathrm{H}), 3.01(\mathrm{~s}, 3 \mathrm{H}), 1.73(\mathrm{~m}, 2 \mathrm{H}), 1.48(\mathrm{~m}, 2 \mathrm{H}), 1.10(\mathrm{t}, J=7.0 \mathrm{~Hz}, 3 \mathrm{H}), 0.98(\mathrm{t}, J=7.1 \mathrm{~Hz}, 3 \mathrm{H})$. ${ }^{13} \mathrm{C}\left\{{ }^{1} \mathrm{H}\right\} \mathrm{NMR}\left(75 \mathrm{MHz}, \mathrm{CDCl}_{3}\right): \delta 169.7,166.8,157.4,149.8,141.3,137.6,135.8,133.8,130.6,130.0$, 127.5, 126.9, 126.3, 122.0, 120.8, 64.5, 61.3, 60.7, 32.6, 31.4, 30.8, 19.2, 13.9, 13.8. ESI-HRMS: Calcd for $\mathrm{C}_{24} \mathrm{H}_{31} \mathrm{~N}_{2} \mathrm{O}_{6} \mathrm{~S}[\mathrm{M}+\mathrm{H}]^{+}$: 475.1897. Found: 475.1891. HPLC: Daicel Chiralpak AD, hexaneisopropanol 90-10, flow rate $0.7 \mathrm{~mL} / \mathrm{min}(\ddot{\mathrm{e}}=254 \mathrm{~nm}), t_{\mathrm{R}}: 28.0 \mathrm{~min}(3 R)-\mathbf{2}$ and $33.8 \mathrm{~min}(3 S)-\mathbf{2}$.

$( \pm)-(E)$-methyl 3-(2-(3-ethoxy-2-( $N$-methylpyridine-2-sulfonamido)-3-oxopropyl)phenyl)acrylate $[( \pm)-3]$ Following the typical procedure, $( \pm)-1(52.3 \mathrm{mg}, 0.15 \mathrm{mmol})$ reacted with methyl acrylate $(27.0$ $\mu \mathrm{L}, \quad 0.30 \mathrm{mmol})$ in the presence of $\mathrm{Pd}(\mathrm{OAc})_{2}(3.4 \mathrm{mg}, 0.015 \mathrm{mmol})$, and 1-fluoro-2,4,6trimethylpyridinium triflate $(130.2 \mathrm{mg}, 0.45 \mathrm{mmol})$ in acetic acid $(1 \mathrm{~mL})$, to afford, after purification by column chromatography ( $n$-hexane-EtOAc 85:15), product ( \pm )-3 as yellow oil; yield: $49.5 \mathrm{mg}(77 \%)$.

${ }^{1} \mathrm{H}$ NMR $\left(300 \mathrm{MHz}, \mathrm{CDCl}_{3}\right): \delta 8.55(\mathrm{~d}, J=4.5 \mathrm{~Hz}, 1 \mathrm{H}), 7.96(\mathrm{~d}, J=15.9 \mathrm{~Hz}, 1 \mathrm{H}), 7.80-7.70(\mathrm{~m}, 3 \mathrm{H})$, $7.45(\mathrm{~d}, J=7.7 \mathrm{~Hz}, 1 \mathrm{H}), 7.35(\mathrm{~m}, 1 \mathrm{H}), 7.25-7.15(\mathrm{~m}, 2 \mathrm{H}), 6.29(\mathrm{~d}, J=15.9 \mathrm{~Hz}, 1 \mathrm{H}), 4.84(\mathrm{~m}, 1 \mathrm{H}), 3.88$ $(\mathrm{dc}, J=2.2,7.0 \mathrm{~Hz}, 2 \mathrm{H}), 3.70(\mathrm{~s}, 3 \mathrm{H}), 3.31(\mathrm{dd}, J=7.0,14.0 \mathrm{~Hz}, 1 \mathrm{H}), 3.06(\mathrm{dd}, J=7.0,14.0 \mathrm{~Hz}, 1 \mathrm{H})$, $3.01(\mathrm{~s}, 3 \mathrm{H}), 0.99(\mathrm{t}, J=7.1 \mathrm{~Hz}, 3 \mathrm{H}) .{ }^{13} \mathrm{C}\left\{{ }^{1} \mathrm{H}\right\} \mathrm{NMR}\left(75 \mathrm{MHz}, \mathrm{CDCl}_{3}\right): \delta 169.7,167.1,157.4,149.8$, $141.6,137.7,135.9,133.8,130.7,130.1,127.6,126.9,126.3,122.1,120.4,61.3,60.8,51.7,32.7,31.4$, 13.9. ESI-HRMS: Calcd for $\mathrm{C}_{21} \mathrm{H}_{25} \mathrm{~N}_{2} \mathrm{O}_{6} \mathrm{~S}[\mathrm{M}+\mathrm{H}]^{+}$: 433.1428. Found: 433.1420 . 
Revised manuscript. Article published in J. Org. Chem. 2015, 80, 3321-3331 (DOI: $10.1021 / j 0502912 \mathrm{~m}$ )

$( \pm)-(E)-E t h y l$

3-(2-(2-(dimethoxyphosphoryl)vinyl)phenyl)-2-(N-methylpyridine-2-

sulfonamido)propanoate $[( \pm)-4]$. Following the typical procedure, $( \pm)-\mathbf{1}(52.3 \mathrm{mg}, 0.15 \mathrm{mmol})$ reacted with dimethyl vinylphosphonate $(35.6 \mu \mathrm{L}, 0.30 \mathrm{mmol})$ in the presence of $\mathrm{Pd}(\mathrm{OAc})_{2}(3.4 \mathrm{mg}, 0.015$ $\mathrm{mmol})$, and 1-fluoro-2,4,6-trimethylpyridinium triflate $(130.2 \mathrm{mg}, 0.45 \mathrm{mmol})$ in acetic acid $(1 \mathrm{~mL})$, to afford, after purification by column chromatography ( $n$-hexane-EtOAc 50:50), product $( \pm)-4$ as yellow oil, yield: $49.3 \mathrm{mg}(68 \%)$.

${ }^{1} \mathrm{H}$ NMR $\left(300 \mathrm{MHz}, \mathrm{CDCl}_{3}\right): \delta 8.56(\mathrm{~m}, 1 \mathrm{H}), 7.82-7.74(\mathrm{~m}, 3 \mathrm{H}), 7.44(\mathrm{dd}, J=1.9,7.7 \mathrm{~Hz}, 1 \mathrm{H}), 7.36$ $(\mathrm{m}, 1 \mathrm{H}), 7.25-7.15(\mathrm{~m}, 3 \mathrm{H}), 6.12(\mathrm{t}, J=7.7 \mathrm{~Hz}, 1 \mathrm{H}), 4.88(\mathrm{~m}, 1 \mathrm{H}), 3.73(\mathrm{~d}, J=5.0 \mathrm{~Hz}, 2 \mathrm{H}), 3.71(\mathrm{~d}, J=$ $4.8 \mathrm{~Hz}, 6 \mathrm{H}), 3.31(\mathrm{dd}, J=6.6,14.2 \mathrm{~Hz}, 1 \mathrm{H}), 3.07(\mathrm{dd}, J=8.5,14.2 \mathrm{~Hz}, 1 \mathrm{H}), 3.01(\mathrm{~s}, 3 \mathrm{H}), 0.98(\mathrm{t}, J=$ $7.3 \mathrm{~Hz}, 3 \mathrm{H}) .{ }^{13} \mathrm{C}\left\{{ }^{1} \mathrm{H}\right\}$ NMR $\left(75 \mathrm{MHz}, \mathrm{CDCl}_{3}\right): \delta 169.7,157.3,149.9,146.3(\mathrm{~d}, J c-p=5.0 \mathrm{~Hz}), 137.7$, $135.5,134.4\left(\mathrm{~d}, J_{c-p}=43.7 \mathrm{~Hz}\right), 130.7,130.2,127.6,126.8,126.4,122.1,115.5\left(\mathrm{~d}, J_{c-p}=160.6 \mathrm{~Hz}\right)$, 61.3, 60.7, 52.6, 32.7, 31.4, 13.9. ESI-HRMS: Calcd for $\mathrm{C}_{21} \mathrm{H}_{28} \mathrm{~N}_{2} \mathrm{O}_{7} \mathrm{PS}[\mathrm{M}+\mathrm{H}]^{+}$: 483.1349. Found: 483.1356.

\section{(S,E)-ethyl 2-(N-methylpyridine-2-sulfonamido)-3-(2-(2-(phenylsulfonyl)vinyl)phenyl)-}

propanoate $[(S)-5]$. Following the typical procedure, $(S)-1(52.3 \mathrm{mg}, 0.15 \mathrm{mmol})$ reacted with phenyl vinyl sulfone $(50.46 \mathrm{mg}, 0.30 \mathrm{mmol})$ in the presence of $\mathrm{Pd}(\mathrm{OAc})_{2}(3.4 \mathrm{mg}, 0.015 \mathrm{mmol})$, and 1-fluoro2,4,6-trimethylpyridinium triflate $(130.2 \mathrm{mg}, 0.45 \mathrm{mmol})$ in acetic acid $(1 \mathrm{~mL})$, to afford, after purification by column chromatography ( $n$-hexane-EtOAc $80: 20)$, product $(S)$-5 as yellow oil, yield: $61.1 \mathrm{mg}(79 \%)$.

$[\alpha]_{\mathrm{D}}^{20}=-11.0\left(c 2.0, \mathrm{CHCl}_{3}\right) ;{ }^{1} \mathrm{H} \mathrm{NMR}\left(300 \mathrm{MHz}, \mathrm{CDCl}_{3}\right): \delta 8.57(\mathrm{dd}, J=0.6,4.5 \mathrm{~Hz}, 1 \mathrm{H}), 7.98(\mathrm{~d}, J$ $=15.0 \mathrm{~Hz}, 1 \mathrm{H}), 7.93(\mathrm{~d}, J=7.9 \mathrm{~Hz}, 2 \mathrm{H}), 7.75-7.70(\mathrm{~m}, 2 \mathrm{H}), 7.55-7.40(\mathrm{~m}, 3 \mathrm{H}), 7.40-7.35(\mathrm{~m}, 2 \mathrm{H})$, 7.30-7.10 (m, 3H), $6.73(\mathrm{~d}, J=15.0 \mathrm{~Hz}, 1 \mathrm{H}), 4.80(\mathrm{t}, J=8.0 \mathrm{~Hz}, 1 \mathrm{H}), 3.95-3.80(\mathrm{~m}, 2 \mathrm{H}), 3.33(\mathrm{dd}, J=$ $7.3 \mathrm{~Hz}, 1 \mathrm{H}), 3.10(\mathrm{dd}, J=7.3 \mathrm{~Hz}, 1 \mathrm{H}), 3.01(\mathrm{~s}, 3 \mathrm{H}), 0.98(\mathrm{t}, J=7.1 \mathrm{~Hz}, 3 \mathrm{H}) .{ }^{13} \mathrm{C}\left\{{ }^{1} \mathrm{H}\right\} \mathrm{NMR}(75 \mathrm{MHz}$, $\left.\mathrm{CDCl}_{3}\right): \delta 169.5,157.2,149.9,140.5,139.4,137.7,136.6,133.8,131.7,131.1,131.0,129.6,129.4$, 
Revised manuscript. Article published in J. Org. Chem. 2015, 80, 3321-3331 (DOI: 10.1021/jo502912m)

$127.9,127.7,127.4,126.5,122.1,61.4,61.0,32.7,31.4,13.9$. ESI-HRMS: Calcd for $\mathrm{C}_{25} \mathrm{H}_{27} \mathrm{~N}_{2} \mathrm{O}_{6} \mathrm{~S}_{2}$ $[\mathrm{M}+\mathrm{H}]^{+}:$515.1305. Found: 515.1310. HPLC: Daicel Chiralpak AD, hexane-isopropanol 85-15, flow rate $0.7 \mathrm{~mL} / \min (\ddot{\mathrm{e}}=254 \mathrm{~nm}), t_{\mathrm{R}}: 87.0 \mathrm{~min}(3 S)-5$ and $93.4 \min (3 R)-5$.

$( \pm)-(E)$-ethyl 2-(N-methylpyridine-2-sulfonamido)-3-(2-(2-(phenoxysulfonyl)vinyl)phenyl)propanoate $[( \pm)-6]$. Following the typical procedure, $( \pm)-1(52.3 \mathrm{mg}, 0.15 \mathrm{mmol})$ reacted with phenyl vinyl sulfonate $(55.2 \mathrm{mg}, 0.30 \mathrm{mmol})$ in the presence of $\mathrm{Pd}(\mathrm{OAc})_{2}(3.4 \mathrm{mg}, 0.015 \mathrm{mmol})$, and 1-fluoro2,4,6-trimethylpyridinium triflate $(130.2 \mathrm{mg}, 0.45 \mathrm{mmol})$ in acetic acid $(1 \mathrm{~mL})$, to afford, after purification by column chromatography ( $n$-hexane-EtOAc $80: 20)$, product $( \pm)-6$ as yellow oil, yield: $58.1 \mathrm{mg}(73 \%)$.

${ }^{1} \mathrm{H}$ NMR (300 MHz, $\left.\mathrm{CDCl}_{3}\right): \delta 8.55(\mathrm{~m}, 1 \mathrm{H}), 7.83(\mathrm{~d}, J=15.1 \mathrm{~Hz}, 1 \mathrm{H}), 7.76-7.48(\mathrm{~m}, 2 \mathrm{H}), 7.45-7.20$ $(\mathrm{m}, 10 \mathrm{H}), 6.73(\mathrm{~d}, J=15.1 \mathrm{~Hz}, 1 \mathrm{H}), 4.77(\mathrm{dd}, J=6.3,8.8 \mathrm{~Hz}, 1 \mathrm{H}), 3.88(\mathrm{dc}, J=1.6,7.6 \mathrm{~Hz}, 2 \mathrm{H}), 3.21$ $(\mathrm{dd}, J=6.3,14.5 \mathrm{~Hz}, 1 \mathrm{H}), 2.99-2.90(\mathrm{~m}, 4 \mathrm{H}), 0.98(\mathrm{t}, J=7.1 \mathrm{~Hz}, 3 \mathrm{H}) .{ }^{13} \mathrm{C}\left\{{ }^{1} \mathrm{H}\right\} \mathrm{NMR}(75 \mathrm{MHz}$, $\left.\mathrm{CDCl}_{3}\right): \delta 169.3,157.2,149.8,149.6,143.3,137.7,136.9,131.4,131.3,131.0,129.9,127.9,127.4$ 127.2, 126.4, 123.1, 122.5, 122.1, 61.5, 60.9, 32.4, 31.9, 13.9. ESI-HRMS: Calcd for $\mathrm{C}_{25} \mathrm{H}_{27} \mathrm{~N}_{2} \mathrm{O}_{7} \mathrm{~S}_{2}$ $[\mathrm{M}+\mathrm{H}]^{+}:$531.1254. Found: 531.1259 .

$( \pm)-(E)$-ethyl 2-( $N$-methylpyridine-2-sulfonamido)-3-(2-(3-oxobut-1-en-1-yl)phenyl)propanoate $[( \pm)-7]$. Following the typical procedure, $( \pm)-\mathbf{1}(52.3 \mathrm{mg}, 0.15 \mathrm{mmol})$ reacted with 3-buten-2-one $(24.3$ $\mu \mathrm{L}, \quad 0.30 \mathrm{mmol})$ in the presence of $\mathrm{Pd}(\mathrm{OAc})_{2}(3.4 \mathrm{mg}, 0.015 \mathrm{mmol})$, and 1-fluoro-2,4,6trimethylpyridinium triflate $(130.2 \mathrm{mg}, 0.45 \mathrm{mmol})$ in acetic acid $(1 \mathrm{~mL})$, to afford, after purification by column chromatography (diethyl ether-hexane 50:50), product $( \pm$ )-7 as yellow oil, yield: $26.5 \mathrm{mg}$ $(43 \%)$.

${ }^{1} \mathrm{H}$ NMR (300 MHz, CDCl 3$): 8.62(\mathrm{dt}, J=4.7,1.4 \mathrm{~Hz}, 1 \mathrm{H}), 7.94(\mathrm{~d}, J=16.1 \mathrm{~Hz}, 1 \mathrm{H}), 7.87-7.81(\mathrm{~m}$, 2H), $7.61-7.55(\mathrm{~m}, 1 \mathrm{H}), 7.44(\mathrm{q}, J=4.6 \mathrm{~Hz}, 1 \mathrm{H}), 7.31-7.22(\mathrm{~m}, 2 \mathrm{H}), 6.64(\mathrm{~d}, J=16.1 \mathrm{~Hz}, 1 \mathrm{H}), 4.90$ $(\mathrm{dd}, J=8.3,6.9 \mathrm{~Hz}, 1 \mathrm{H}), 3.90(\mathrm{q}, J=7.1 \mathrm{~Hz}, 2 \mathrm{H}), 3.40(\mathrm{dd}, J=14.0,8.3 \mathrm{~Hz}, 1 \mathrm{H}), 3.23(\mathrm{dd}, J=14.0$, 
Revised manuscript. Article published in J. Org. Chem. 2015, 80, 3321-3331 (DOI: 10.1021/jo502912m)

$6.9 \mathrm{~Hz}, 1 \mathrm{H}), 3.09$ (s, 3H), $2.44(\mathrm{~s}, 3 \mathrm{H}), 0.98(\mathrm{t}, J=7.2 \mathrm{~Hz}, 3 \mathrm{H}) .{ }^{13} \mathrm{C}\left\{{ }^{1} \mathrm{H}\right\} \mathrm{NMR}\left(75 \mathrm{MHz}, \mathrm{CDCl}_{3}\right): \delta$ $199.1,169.6,157.3,149.9,140.3,137.9,136.0,134.1,131.3,130.2,130.1,127.9,127.0,126.6,122.4$, 61.3, 60.7, 33.7, 31.4, 27.4, 14.0. ESI-HRMS: Calcd for $\mathrm{C}_{21} \mathrm{H}_{24} \mathrm{~N}_{2} \mathrm{O}_{5} \mathrm{~S}[\mathrm{M}+\mathrm{H}]^{+}$: 417.1479. Found: 417.1510.

( \pm )-(E)-ethyl 2-( $N$-methylpyridine-2-sulfonamido)-3-(2-(3-oxoprop-1-en-1-yl)phenyl)propanoate $[( \pm)-8]$. Following the typical procedure, $( \pm)-1(52.3 \mathrm{mg}, 0.15 \mathrm{mmol})$ reacted with acrolein $(20.1 \mu \mathrm{L}$ $0.30 \mathrm{mmol})$ in the presence of $\mathrm{Pd}(\mathrm{OAc})_{2}(3.4 \mathrm{mg}, 0.015 \mathrm{mmol})$, and 1-fluoro-2,4,6-trimethylpyridinium triflate $(130.2 \mathrm{mg}, 0.45 \mathrm{mmol})$ in acetic acid $(1 \mathrm{~mL})$, to afford, after purification by column chromatography (diethyl ether-hexane 50:50), product ( \pm )-8 as yellow oil, yield: $15.3 \mathrm{mg}(25 \%)$.

${ }^{1} \mathrm{H}$ NMR (300 MHz, CDCl3): 9.77 (d, $\left.J=7.7 \mathrm{~Hz}, 1 \mathrm{H}\right), 8.63$ (d, $\left.J=4.3 \mathrm{~Hz}, 1 \mathrm{H}\right), 8.05$ (d, $J=15.7 \mathrm{~Hz}$, 1H), $7.91-7.84(\mathrm{~m}, 2 \mathrm{H}), 7.66-7.60(\mathrm{~m}, 1 \mathrm{H}), 7.46(\mathrm{td}, J=5.1,3.0 \mathrm{~Hz}, 1 \mathrm{H}), 7.36-7.27(\mathrm{~m}, 3 \mathrm{H}), 6.69$ $(\mathrm{dd}, J=15.7,7.7 \mathrm{~Hz}, 1 \mathrm{H}), 4.90(\mathrm{dd}, J=8.9,6.1 \mathrm{~Hz}, 1 \mathrm{H}), 3.92(\mathrm{qd}, J=7.1,1.0 \mathrm{~Hz}, 2 \mathrm{H}), 3.43(\mathrm{dd}, J=$ 13.9, 8.9 Hz, 1H), $3.28(\mathrm{dd}, J=13.9,6.2 \mathrm{~Hz}, 1 \mathrm{H}), 3.08(\mathrm{~s}, 3 \mathrm{H}), 0.98(\mathrm{t}, J=7.1 \mathrm{~Hz}, 3 \mathrm{H}) .{ }^{13} \mathrm{C}\left\{{ }^{1} \mathrm{H}\right\} \mathrm{NMR}$ $\left(75 \mathrm{MHz}, \mathrm{CDCl}_{3}\right): \delta 94.3,169.5,157.3,149.9,149.4,137.9,136.3,133.5,131.6,131.0,130.8,128.0$, 127.2, 126.7, 122.5, 61.4, 60.8, 33.8, 31.4, 14.0. ESI-HRMS: Calcd for $\mathrm{C}_{20} \mathrm{H}_{23} \mathrm{~N}_{2} \mathrm{O} \mathrm{O}_{5} \mathrm{~S}[\mathrm{M}+\mathrm{H}]^{+}$: 403.1328. Found: 403.1322.

\section{$( \pm)-(E)-E t h y l \quad 2-(N$-methylpyridine-2-sulfonamido)-3-(2-(2-(perfluorophenyl)vinyl)phenyl)-}

propanoate $[( \pm)-9]$. Following the typical procedure, $( \pm)-1(52.3 \mathrm{mg}, 0.15 \mathrm{mmol})$ reacted with 2,3,4,5,6-pentafluorostyrene $(41.4 \mu \mathrm{L}, 0.30 \mathrm{mmol})$ in the presence of $\mathrm{Pd}(\mathrm{OAc}) 2(3.4 \mathrm{mg}, 0.015 \mathrm{mmol})$, and 1-fluoro-2,4,6-trimethylpyridinium triflate $(130.2 \mathrm{mg}, 0.45 \mathrm{mmol})$ in acetic acid $(1 \mathrm{~mL})$, to afford, after purification by column chromatography (diethyl ether-hexane 50:50), product ( \pm )-9 as yellow oil, yield: $45.0 \mathrm{mg}(56 \%)$.

${ }^{1} \mathrm{H}$ NMR $\left(300 \mathrm{MHz}, \mathrm{CDCl}_{3}\right): 8.58(\mathrm{~s}, 1 \mathrm{H}), 7.83-7.71(\mathrm{~m}, 2 \mathrm{H}), 7.76(\mathrm{~d}, J=16.5 \mathrm{~Hz}, 1 \mathrm{H}), 7.56(\mathrm{dd}, J$ $=6.9,1.8 \mathrm{~Hz}, 1 \mathrm{H}), 7.44-7.37(\mathrm{~m}, 1 \mathrm{H}), 7.29-7.20(\mathrm{~m}, 3 \mathrm{H}), 6.86(\mathrm{~d}, J=16.5 \mathrm{~Hz}, 1 \mathrm{H}), 4.94(\mathrm{dd}, J=$ 
Revised manuscript. Article published in J. Org. Chem. 2015, 80, 3321-3331 (DOI: $10.1021 / j 0502912 \mathrm{~m}$ )

8.5, $6.7 \mathrm{~Hz}, 1 \mathrm{H}), 3.96(\mathrm{q}, J=7.1 \mathrm{~Hz}, 2 \mathrm{H}), 3.42(\mathrm{dd}, J=14.3,6.7 \mathrm{~Hz}, 1 \mathrm{H}), 3.11(\mathrm{dd}, J=14.3,6.7 \mathrm{~Hz}$, 1H), $3.11(\mathrm{~s}, 3 \mathrm{H}), 1.06(\mathrm{t}, J=7.1 \mathrm{~Hz}, 3 \mathrm{H}) .{ }^{13} \mathrm{C}\left\{{ }^{1} \mathrm{H}\right\}$ NMR $\left(75 \mathrm{MHz}, \mathrm{CDCl}_{3}\right): \delta 169.8,157.3,149.6$, $144.8\left(\mathrm{q}, J_{C-F}=252.12 \mathrm{~Hz}\right), 139.8\left(\mathrm{q}, J_{C-F}=252.12 \mathrm{~Hz}\right), 137.6,137.6\left(\mathrm{q}, J_{C-F}=252.12 \mathrm{~Hz}\right), 136.0$, $134.7,134.5\left(\mathrm{td}, J_{C-F}=8.1,2.4 \mathrm{~Hz}\right), 130.6,128.8,127.6,126.3,126.1,121.9,115.3\left(\mathrm{~d}, J_{C-F}=3.2 \mathrm{~Hz}\right)$, $112.43(\mathrm{~m}), 61.2,60.5,33.2,31.3,13.8 .{ }^{19} \mathrm{~F}\left\{{ }^{1} \mathrm{H}\right\} \mathrm{NMR}: \delta-142.55\left(\mathrm{dd}, J_{C-F}=21.2,7.6 \mathrm{~Hz}, 2 \mathrm{~F}\right),-156.30$ $\left(\mathrm{t}, J_{C-F}=20.8 \mathrm{~Hz}, 1 \mathrm{~F}\right),-162.94\left(\mathrm{td}, J_{C-F}=21.2,7.6 \mathrm{~Hz}, 2 \mathrm{~F}\right)$. ESI-HRMS: Calcd for $\mathrm{C}_{25} \mathrm{H}_{22} \mathrm{~F}_{5} \mathrm{~N}_{2} \mathrm{O}_{4} \mathrm{~S}$ $[\mathrm{M}+\mathrm{H}]^{+}:$541.1215. Found: 541.1251 .

( \pm )-(E)-Ethyl 2-(N-methylpyridine-2-sulfonamido)-3-(2-(3-nitrostyryl)phenyl)propanoate $[( \pm)-$ 10]. Following the typical procedure, $( \pm)-1(52.3 \mathrm{mg}, 0.15 \mathrm{mmol})$ reacted with 3 -nitrostyrene $(41.8 \mu \mathrm{L}$, $0.30 \mathrm{mmol})$ in the presence of $\mathrm{Pd}(\mathrm{OAc}) 2(3.4 \mathrm{mg}, 0.015 \mathrm{mmol})$, and 1-fluoro-2,4,6-trimethylpyridinium triflate $(130.2 \mathrm{mg}, 0.45 \mathrm{mmol})$ in acetic acid $(1 \mathrm{~mL})$, to afford, after purification by column chromatography (diethyl ether-hexane 50:50), product ( \pm )-10 as yellow oil, yield: $36.3 \mathrm{mg}(49 \%)$.

${ }^{1} \mathrm{H}$ NMR $\left(300 \mathrm{MHz}, \mathrm{CDCl}_{3}\right): 8.58(\mathrm{dt}, J=4.7,1.4 \mathrm{~Hz}, 1 \mathrm{H}), 8.38(\mathrm{t}, J=2.1 \mathrm{~Hz}, 1 \mathrm{H}), 8.10-8.05(\mathrm{~m}$, 1H), $7.95(\mathrm{dt}, J=8.0,1.5 \mathrm{~Hz}, 1 \mathrm{H}), 7.84-7.80(\mathrm{~m}, 2 \mathrm{H}), 7.71(\mathrm{~d}, J=16.0 \mathrm{~Hz}, 1 \mathrm{H}), 7.63(\mathrm{~d}, J=7.6 \mathrm{~Hz}$, 1H), $7.49(\mathrm{t}, J=7.9 \mathrm{~Hz}, 1 \mathrm{H}), 7.45-7.38(\mathrm{~m}, 1 \mathrm{H}), 7.30-7.24(\mathrm{~m}, 1 \mathrm{H}), 7.22-7.18(\mathrm{~m}, 2 \mathrm{H}), 7.08(\mathrm{~d}, J=$ $15.8 \mathrm{~Hz}, 1 \mathrm{H}), 5.00(\mathrm{dd}, J=8.5,6.7 \mathrm{~Hz}, 1 \mathrm{H}), 3.94(\mathrm{q}, J=7.1 \mathrm{~Hz}, 2 \mathrm{H}), 3.40(\mathrm{dd}, J=13.8,8.5 \mathrm{~Hz}, 1 \mathrm{H})$, $3.28(\mathrm{dd}, J=13.8,6.7 \mathrm{~Hz}, 1 \mathrm{H}), 3.10(\mathrm{~s}, 3 \mathrm{H}), 1.00(\mathrm{t}, J=7.1 \mathrm{~Hz}, 3 \mathrm{H}) .{ }^{13} \mathrm{C}\left\{{ }^{1} \mathrm{H}\right\} \mathrm{NMR}\left(75 \mathrm{MHz}, \mathrm{CDCl}_{3}\right)$ : $\delta 169.8,157.2,149.8,148.9,139.3,137.8,136.0,134.5,132.6,131.1,129.6,129.0,128.7,128.4,127.8$, 126.6, 126.2, $122.5,122.2,121.7,61.2,60.5,34.0,31.2$, 14.0. ESI-HRMS: Calcd for $\mathrm{C}_{25} \mathrm{H}_{26} \mathrm{~N}_{3} \mathrm{O}_{6} \mathrm{~S}$ $[\mathrm{M}+\mathrm{H}]^{+}:$496.1537. Found: 496.1541.

\section{( \pm -(E)-ethyl 2-(N-methylpyridine-2-sulfonamido)-3-(2-(3-(trifluoromethyl)styryl)phenyl)-}

propanoate $[( \pm)-11]$. Following the typical procedure, $( \pm)-1(52.3 \mathrm{mg}, 0.15 \mathrm{mmol})$ reacted with 3 (trifluoromethyl)styrene $(44.5 \mu \mathrm{L}, 0.30 \mathrm{mmol})$ in the presence of $\mathrm{Pd}(\mathrm{OAc})_{2}(3.4 \mathrm{mg}, 0.015 \mathrm{mmol})$, and 1-fluoro-2,4,6-trimethylpyridinium triflate $(130.2 \mathrm{mg}, 0.45 \mathrm{mmol})$ in acetic acid $(1 \mathrm{~mL})$, to afford, after 
Revised manuscript. Article published in J. Org. Chem. 2015, 80, 3321-3331 (DOI: $10.1021 / j 0502912 \mathrm{~m}$ )

purification by column chromatography (diethyl ether-hexane 50:50), product $( \pm$ )-11 as yellow oil, yield: $28.7 \mathrm{mg}(37 \%)$.

${ }^{1} \mathrm{H}$ NMR $\left(300 \mathrm{MHz}, \mathrm{CDCl}_{3}\right): 8.56(\mathrm{~s}, 1 \mathrm{H}), 7.86-7.73(\mathrm{~m}, 4 \mathrm{H}), 7.62(\mathrm{~d}, J=15.6 \mathrm{~Hz}, 1 \mathrm{H}), 7.61(\mathrm{~d}, J=$ $8.4 \mathrm{~Hz}, 1 \mathrm{H}), 7.52-7.48(\mathrm{~m}, 1 \mathrm{H}), 7.48-7.43(\mathrm{~m}, 1 \mathrm{H}), 7.43-7.35(\mathrm{~m}, 1 \mathrm{H}), 7.31-7.23(\mathrm{~m}, 1 \mathrm{H}), 7.21-$ $7.16(\mathrm{~m}, 2 \mathrm{H}), 7.05(\mathrm{~d}, J=16.1 \mathrm{~Hz}, 1 \mathrm{H}), 5.00(\mathrm{dd}, J=8.3,6.9 \mathrm{~Hz}, 1 \mathrm{H}), 3.93(\mathrm{q}, J=7.1 \mathrm{~Hz}, 2 \mathrm{H}), 3.39$ $(\mathrm{dd}, J=13.9,8.3 \mathrm{~Hz}, 1 \mathrm{H}), 3.26(\mathrm{dd}, J=13.8,6.8 \mathrm{~Hz}, 1 \mathrm{H}), 3.11(\mathrm{~s}, 3 \mathrm{H}), 1.00$ (t, $J=7.1 \mathrm{~Hz}, 3 \mathrm{H})$. $\left.{ }^{13} \mathrm{C}\left\{{ }^{1} \mathrm{H}\right\} \operatorname{NMR}\left(75 \mathrm{MHz}, \mathrm{CDCl}_{3}\right): \delta 169.93,157.30,149.83,138.29,137.79\right), 136.38,134.33,131.09$ (q, $\left.J_{C-F}=32.24 \mathrm{~Hz}\right), 131.05,129.99,129.20,128.14,127.71,127.52,126.54,126.17,124.25\left(\mathrm{q}, J_{C-F}=3.8\right.$ $\mathrm{Hz}), 123.77\left(\mathrm{q}, J_{C-F}=3.77 \mathrm{~Hz}\right), 122.44,61.23,60.47,33.93,31.31,13.97$. The $\mathrm{CF}_{3}$ carbon was not observed, in spite of the use of long accumulation trials. ${ }^{19} \mathrm{~F}\left\{{ }^{1} \mathrm{H}\right\}$ NMR: $\delta-62.75$. ESI-HRMS: Calcd for $\mathrm{C}_{26} \mathrm{H}_{25} \mathrm{~F}_{3} \mathrm{~N}_{2} \mathrm{O}_{4} \mathrm{~S}[\mathrm{M}+\mathrm{H}]^{+}:$519.1560. Found: 519.1578 .

$( \pm)-(E)-B u t y l$ 3-(2-(3-ethoxy-2-( $N$-methylpyridine-2-sulfonamido)-3-oxopropyl)-5methoxyphenyl)-acrylate $[( \pm)-12]$. Following the typical procedure, sulfonamide $( \pm)$-XIX (56.8 mg, $0.15 \mathrm{mmol})$ reacted with butyl acrylate $(43.0 \mu \mathrm{L}, 0.30 \mathrm{mmol})$ in the presence of $\mathrm{Pd}(\mathrm{OAc})_{2}(3.4 \mathrm{mg}$, $0.015 \mathrm{mmol})$, and 1-fluoro-2,4,6-trimethylpyridinium triflate $(130.2 \mathrm{mg}, 0.45 \mathrm{mmol})$ in acetic acid (1 $\mathrm{mL}$ ), to afford, after purification by column chromatography ( $n$-hexane-EtOAc 80:20), product $( \pm)-\mathbf{1 2}$ as yellow oil, yield: $53.7 \mathrm{mg}(71 \%)$.

${ }^{1} \mathrm{H}$ NMR $\left(300 \mathrm{MHz}, \mathrm{CDCl}_{3}\right): \delta=8.54(\mathrm{~d}, J=4.5 \mathrm{~Hz}, 1 \mathrm{H}), 7.90(\mathrm{~d}, J=15.6 \mathrm{~Hz}, 1 \mathrm{H}), 7.80-7.65(\mathrm{~m}$, 2H), $7.35(\mathrm{~m}, 1 \mathrm{H}), 7.07(\mathrm{~d}, J=8.3 \mathrm{~Hz}, 1 \mathrm{H}), 6.94(\mathrm{~d}, J=2.6 \mathrm{~Hz}, 1 \mathrm{H}), 6.75(\mathrm{dd}, J=2.6,8.3 \mathrm{~Hz}, 1 \mathrm{H})$, $6.26(\mathrm{~d}, J=15.6 \mathrm{~Hz}, 1 \mathrm{H}), 4.79(\mathrm{dd}, J=2.8,8.7 \mathrm{~Hz}, 1 \mathrm{H}), 4.14(\mathrm{t}, J=6.6 \mathrm{~Hz}, 2 \mathrm{H}), 3.90(\mathrm{dc}, J=1.0,7.9$ $\mathrm{Hz}, 2 \mathrm{H}), 3.73(\mathrm{~s}, 3 \mathrm{H}), 3.25(\mathrm{dd}, J=6.6,14.1 \mathrm{~Hz}, 1 \mathrm{H}), 3.01(\mathrm{~m}, 4 \mathrm{H}), 1.62(\mathrm{~m}, 2 \mathrm{H}), 1.36(\mathrm{~m}, 2 \mathrm{H}), 1.00(\mathrm{t}$, $J=7.1 \mathrm{~Hz}, 3 \mathrm{H}), 0.89(\mathrm{t}, J=7.1 \mathrm{~Hz}, 3 \mathrm{H}) .{ }^{13} \mathrm{C}\left\{{ }^{1} \mathrm{H}\right\} \mathrm{NMR}\left(75 \mathrm{MHz}, \mathrm{CDCl}_{3}\right): \delta 169.7,166.7,158.7,157.5$, $149.7,141.3,137.6,134.8,131.8,128.1,126.2,122.0,120.8,116.1,111.5,64.5,61.2,60.9,55.3,31.9$, 31.4, 30.8, 19.2, 13.9, 13.7. ESI-HRMS: Calcd for $\mathrm{C}_{25} \mathrm{H}_{33} \mathrm{~N}_{2} \mathrm{O}_{7} \mathrm{~S}[\mathrm{M}+\mathrm{H}]^{+}$, 505.2003. Found 505.2009. 
Revised manuscript. Article published in J. Org. Chem. 2015, 80, 3321-3331 (DOI: 10.1021/jo502912m)

$( \pm)-(E)-B u t y l$

3-(2-(3-ethoxy-2-( $N$-methylpyridine-2-sulfonamido)-3-oxopropyl)-5-

methylphenyl)-acrylate $[( \pm)-13]$. Following the typical procedure, sulfonamide $( \pm)-\mathbf{X X}(54.5 \mathrm{mg}, 0.15$ mmol) reacted with butyl acrylate $(43.0 \mu \mathrm{L}, 0.30 \mathrm{mmol})$ in the presence of $\mathrm{Pd}(\mathrm{OAc})_{2}(3.4 \mathrm{mg}, 0.015$ $\mathrm{mmol})$, and 1-fluoro-2,4,6-trimethylpyridinium triflate $(130.2 \mathrm{mg}, 0.45 \mathrm{mmol})$ in acetic acid $(1 \mathrm{~mL})$, to afford, after purification by column chromatography ( $n$-hexane-EtOAc 80:20), product $( \pm)$-13 as yellow oil; yield: $31.3 \mathrm{mg}(43 \%)$.

${ }^{1} \mathrm{H}$ NMR $\left(300 \mathrm{MHz}, \mathrm{CDCl}_{3}\right): \delta 8.61(\mathrm{dt}, J=4.8,1.5 \mathrm{~Hz}, 1 \mathrm{H}), 7.98(\mathrm{~d}, J=15.7 \mathrm{~Hz}, 1 \mathrm{H}), 7.85-7.75$ (m, 2H), $7.42(\mathrm{ddd}, J=6.7,4.7,2.1 \mathrm{~Hz}, 1 \mathrm{H}), 7.33$ (d, $J=1.8 \mathrm{~Hz}, 1 \mathrm{H}), 7.13(\mathrm{~d}, J=7.8 \mathrm{~Hz}, 1 \mathrm{H}), 7.08$ $(\mathrm{dd}, J=7.8,1.8 \mathrm{~Hz}, 1 \mathrm{H}), 6.34(\mathrm{~d}, J=15.7 \mathrm{~Hz}, 1 \mathrm{H}), 4.89(\mathrm{dd}, J=8.8,6.6 \mathrm{~Hz}, 1 \mathrm{H}), 4.21(\mathrm{t}, J=6.7 \mathrm{~Hz}$, 2H), $3.97(\mathrm{qd}, J=7.1,1.4 \mathrm{~Hz}, 2 \mathrm{H}), 3.35(\mathrm{dd}, J=14.4,6.6 \mathrm{~Hz}, 1 \mathrm{H}), 3.08(\mathrm{~s}, 3 \mathrm{H}), 3.07$ (dd, $J=14.4,8.8$ $\mathrm{Hz}, 1 \mathrm{H}), 2.32(\mathrm{~s}, 3 \mathrm{H}), 1.73-1.64(\mathrm{~m}, 2 \mathrm{H}), 1.48-1.40(\mathrm{~m}, 2 \mathrm{H}), 1.07(\mathrm{t}, J=7.1 \mathrm{~Hz}, 3 \mathrm{H}), 0.96(\mathrm{t}, J=7.4$ $\mathrm{Hz}, 3 \mathrm{H}) .{ }^{13} \mathrm{C}\left\{{ }^{1} \mathrm{H}\right\} \mathrm{NMR}\left(75 \mathrm{MHz}, \mathrm{CDCl}_{3}\right): \delta 169.9,167.0,157.5,149.8,141.5,137.7,137.1,133.6$, $133.0,131.0,130.7,127.5,126.3,122.1,120.5,64.6,61.4,60.9,32.3,31.5,30.9,21.2,19.3,14.0,13.9$. ESI-HRMS: Calcd for $\mathrm{C}_{25} \mathrm{H}_{32} \mathrm{~N}_{2} \mathrm{NaO}_{6} \mathrm{~S}[\mathrm{M}+\mathrm{Na}]^{+}:$511.1873. Found: 511.1867.

( \pm )-(E)-Butyl 3-(2-(3-ethoxy-2-( $N$-methylpyridine-2-sulfonamido)-3-oxopropyl)-5-fluorophenyl)acrylate $[( \pm)-14]$. Following the typical procedure, sulfonamide $( \pm)$-XXI (55.0 mg, $0.15 \mathrm{mmol})$ reacted with butyl acrylate $(43.0 \mu \mathrm{L}, 0.30 \mathrm{mmol})$ in the presence of $\mathrm{Pd}(\mathrm{OAc})_{2}(3.4 \mathrm{mg}, 0.015 \mathrm{mmol})$, and 1fluoro-2,4,6-trimethylpyridinium triflate $(130.2 \mathrm{mg}, 0.45 \mathrm{mmol})$ in acetic acid $(1 \mathrm{~mL})$, to afford, after purification by column chromatography ( $n$-hexane-EtOAc $80: 20)$, product $( \pm)$-14 as yellow oil; yield: $29.6 \mathrm{mg}(40 \%)$.

${ }^{1} \mathrm{H}$ NMR (300 MHz, $\left.\mathrm{CDCl}_{3}\right): \delta 8.65(\mathrm{~d}, J=3.7 \mathrm{~Hz}, 1 \mathrm{H}), 7.98(\mathrm{~d}, J=15.8 \mathrm{~Hz}, 1 \mathrm{H}), 7.90-7.80(\mathrm{~m}, 2 \mathrm{H})$, $7.47(\mathrm{~m}, 1 \mathrm{H}), 7.30-7.13(\mathrm{~m}, 2 \mathrm{H}), 7.01(\mathrm{~m}, 1 \mathrm{H}), 6.37(\mathrm{~d}, J=15.8 \mathrm{~Hz}, 1 \mathrm{H}), 4.91(\mathrm{~m}, 1 \mathrm{H}), 4.25(\mathrm{t}, J=6.8$ $\mathrm{Hz}, 2 \mathrm{H}), 4.01(\mathrm{~m}, 2 \mathrm{H}), 3.40(\mathrm{dd}, J=6.4,14.5 \mathrm{~Hz}, 1 \mathrm{H}), 3.16-3.10(\mathrm{~m}, 4 \mathrm{H}), 1.73(\mathrm{~m}, 2 \mathrm{H}), 1.48(\mathrm{~m}, 2 \mathrm{H})$, $1.11(\mathrm{t}, J=7.2 \mathrm{~Hz}, 3 \mathrm{H}), 1.00(\mathrm{t}, J=7.2 \mathrm{~Hz}, 3 \mathrm{H}) .{ }^{13} \mathrm{C}\left\{{ }^{1} \mathrm{H}\right\} \mathrm{NMR}\left(75 \mathrm{MHz}, \mathrm{CDCl}_{3}\right): \delta 169.5,166.4$, 
Revised manuscript. Article published in J. Org. Chem. 2015, 80, 3321-3331 (DOI: $10.1021 / j 0502912 \mathrm{~m}$ )

157.4, 149.8, $140.1(2 \mathrm{C}), 137.7,135.7\left(\mathrm{~d}, J_{C-F}=7.3 \mathrm{~Hz}\right), 132.4\left(\mathrm{~d}, J_{C-F}=7.3 \mathrm{~Hz}\right), 131.7,126.3,122.0$, $121.9,116.9\left(\mathrm{~d}, J_{C-F}=22.0 \mathrm{~Hz}\right), 113.2\left(\mathrm{~d}, J_{C-F}=22.6 \mathrm{~Hz}\right), 64.7,61.4,60.9,32.0,31.4,30.7,19.2,13.9$, 13.7. ${ }^{19} \mathrm{~F}\left\{{ }^{1} \mathrm{H}\right\}$ NMR (282 MHz, $\left.\mathrm{CDCl}_{3}\right): \delta$-114.7. ESI-HRMS: Calcd for $\mathrm{C}_{24} \mathrm{H}_{30} \mathrm{FN}_{2} \mathrm{O} 6 \mathrm{~S}[\mathrm{M}+\mathrm{H}]^{+}$: 493.1803. Found: 493.1805.

( \pm )-(E)-Butyl 3-(5-chloro-2-(3-ethoxy-2-( $N$-methylpyridine-2-sulfonamido)-3-oxopropyl)phenyl)acrylate $[( \pm)-15]$. Following the typical procedure, sulfonamide $( \pm)$-XXII (57.5 mg, $0.15 \mathrm{mmol})$ reacted with butyl acrylate $(43.0 \mu \mathrm{L}, 0.30 \mathrm{mmol})$ in the presence of $\mathrm{Pd}(\mathrm{OAc})_{2}(3.4 \mathrm{mg}, 0.015 \mathrm{mmol})$, and 1fluoro-2,4,6-trimethylpyridinium triflate $(130.2 \mathrm{mg}, 0.45 \mathrm{mmol})$ in acetic acid $(1 \mathrm{~mL})$, to afford, after purification by column chromatography ( $n$-hexane-EtOAc 80:20), product $( \pm)$-15 as yellow oil; yield: $18.2 \mathrm{mg}(24 \%)$. It should be noted that he difficulty in the complete chromatographic separation of the products from the starting material and trace amount of unidentified byproducts resulted in low yields upon isolation.

${ }^{1} \mathrm{H}$ NMR $\left(300 \mathrm{MHz}, \mathrm{CDCl}_{3}\right): \delta 8.60(\mathrm{ddd}, J=4.7,1.7,0.9 \mathrm{~Hz}, 1 \mathrm{H}), 7.91(\mathrm{~d}, J=15.7 \mathrm{~Hz}, 1 \mathrm{H}), 7.82$ $(\mathrm{td}, J=7.7,1.7 \mathrm{~Hz}, 1 \mathrm{H}), 7.76(\mathrm{tt}, J=7.9,1.0 \mathrm{~Hz}, 1 \mathrm{H}), 7.46(\mathrm{~d}, J=2.1 \mathrm{~Hz}, 1 \mathrm{H}), 7.44(\mathrm{ddd}, J=7.6,4.7$, $1.2 \mathrm{~Hz}, 1 \mathrm{H}), 7.20(\mathrm{dd}, J=8.3,2.1 \mathrm{~Hz}, 1 \mathrm{H}), 7.17-7.15(\mathrm{~m}, 1 \mathrm{H}), 6.34(\mathrm{~d}, J=15.7 \mathrm{~Hz}, 1 \mathrm{H}), 4.87(\mathrm{dd}, J=$ 9.0, $6.4 \mathrm{~Hz}, 1 \mathrm{H}), 4.21(\mathrm{t}, J=6.7 \mathrm{~Hz}, 2 \mathrm{H}), 4.03-3.98(\mathrm{~m}, 2 \mathrm{H}), 3.35(\mathrm{dd}, J=14.5,6.4 \mathrm{~Hz}, 1 \mathrm{H}), 3.08(\mathrm{dd}$, $J=14.5,9.0 \mathrm{~Hz}, 1 \mathrm{H}), 3.07(\mathrm{~s}, 3 \mathrm{H}), 1.71-1.65(\mathrm{~m}, 2 \mathrm{H}), 1.46-1.40(\mathrm{~m}, 2 \mathrm{H}), 1.09(\mathrm{t}, J=7.1 \mathrm{~Hz}, 3 \mathrm{H})$, $0.96(\mathrm{t}, J=7.4 \mathrm{~Hz}, 3 \mathrm{H}) .{ }^{13} \mathrm{C}\left\{{ }^{1} \mathrm{H}\right\} \mathrm{NMR}: \delta 169.5,166.5,157.4,149.9,139.9,137.8,135.6,134.4,133.4$ 132.1, 129.9, 126.8, 126.4, 122.1, 122.0, 64.7, 61.6, 60.8, 32.1, 31.5, 30.8, 19.3, 14.0, 13.8. ESI-HRMS: Calcd for $\mathrm{C}_{24} \mathrm{H}_{30} \mathrm{ClN}_{2} \mathrm{O}_{6} \mathrm{~S}[\mathrm{M}+\mathrm{H}]^{+}:$509.1508. Found: 509.1499 .

$( \pm)-(E)-B u t y l$

3-(5-bromo-2-(3-ethoxy-2-( $N$-methylpyridine-2-sulfonamido)-3-

oxopropyl)phenyl)-acrylate $[( \pm)-16]$. Following the typical procedure, sulfonamide $( \pm)$-XXIII $(64.0$ $\mathrm{mg}, 0.15 \mathrm{mmol})$ reacted with butyl acrylate $(43.0 \mu \mathrm{L}, 0.30 \mathrm{mmol})$ in the presence of $\mathrm{Pd}(\mathrm{OAc})_{2}(3.4 \mathrm{mg}$, $0.015 \mathrm{mmol})$, and 1-fluoro-2,4,6-trimethylpyridinium triflate $(130.2 \mathrm{mg}, 0.45 \mathrm{mmol})$ in acetic acid (1 
Revised manuscript. Article published in J. Org. Chem. 2015, 80, 3321-3331 (DOI: $10.1021 / j 0502912 \mathrm{~m}$ )

$\mathrm{mL}$ ), to afford, after purification by column chromatography ( $n$-hexane-EtOAc 80:20), product ( \pm )-16 as yellow oil; yield: $23.8 \mathrm{mg}(29 \%)$. It should be noted that he difficulty in the complete chromatographic separation of the products from the starting material and trace amount of unidentified byproducts resulted in low yields upon isolation.

${ }^{1} \mathrm{H}$ NMR $\left(300 \mathrm{MHz}, \mathrm{CDCl}_{3}\right): \delta 8.60(\mathrm{ddd}, J=4.6,1.6,0.8 \mathrm{~Hz}, 1 \mathrm{H}), 7.90(\mathrm{~d}, J=15.7 \mathrm{~Hz}, 1 \mathrm{H}), 7.82$ (td, $J=7.7,1.7 \mathrm{~Hz}, 1 \mathrm{H}), 7.75(\mathrm{tt}, J=7.9,1.0 \mathrm{~Hz}, 1 \mathrm{H}), 7.61(\mathrm{~d}, J=2.1 \mathrm{~Hz}, 1 \mathrm{H}), 7.44$ (ddd, $J=7.6,4.7$, $1.2 \mathrm{~Hz}, 1 \mathrm{H}), 7.33$ (ddd, $J=8.7,7.4,2.0 \mathrm{~Hz}, 1 \mathrm{H}), 7.10(\mathrm{~d}, J=8.3 \mathrm{~Hz}, 1 \mathrm{H}), 6.33(\mathrm{~d}, J=15.7 \mathrm{~Hz}, 1 \mathrm{H})$, $4.88(\mathrm{dd}, J=9.0,6.3 \mathrm{~Hz}, 1 \mathrm{H}), 4.21(\mathrm{t}, J=6.7 \mathrm{~Hz}, 2 \mathrm{H}), 4.03-3.99(\mathrm{~m}, 2 \mathrm{H}), 3.33(\mathrm{dd}, J=14.5,6.3 \mathrm{~Hz}$, 1H), $3.07(\mathrm{~s}, 3 \mathrm{H}), 3.07(\mathrm{dd}, J=14.5,5.9 \mathrm{~Hz}, 1 \mathrm{H}), 1.73-1.64(\mathrm{~m}, 2 \mathrm{H}), 1.47-1.41(\mathrm{~m}, 2 \mathrm{H}), 1.10(\mathrm{t}, J=$ $7.2 \mathrm{~Hz}, 3 \mathrm{H}), 0.96(\mathrm{t}, J=7.4 \mathrm{~Hz}, 3 \mathrm{H}) .{ }^{13} \mathrm{C}\left\{{ }^{1} \mathrm{H}\right\} \mathrm{NMR}: \delta 169.6,166.5,157.4,149.9,139.8,137.8,135.9$, $134.9,132.8,132.3,129.8,126.4,122.2,122.0,121.5,64.8,61.6,60.7,32.2,31.5,30.8,19.3,14.1$, 13.8. ESI-HRMS: Calcd for $\mathrm{C}_{24} \mathrm{H}_{30} \mathrm{BrN}_{2} \mathrm{O}_{6} \mathrm{~S}[\mathrm{M}+\mathrm{H}]^{+}: 553.1002$. Found: 553.1027.

( \pm )-(E)-Butyl 3-(2-(3-ethoxy-2-( $N$-methylpyridine-2-sulfonamido)-3-oxopropyl)-6-fluorophenyl)acrylate $[( \pm)-17 \mathrm{a}]$ and $( \pm)-(E)$-Butyl 3-(2-(3-ethoxy-2-( $N$-methylpyridine-2-sulfonamido)-3oxopropyl)-4-fluorophenyl)acrylate $[( \pm)-17 b]$. Following the typical procedure, sulfonamide $( \pm)$ XXIV (55.0 mg, $0.15 \mathrm{mmol})$ reacted with butyl acrylate $(43.0 \mu \mathrm{L}, 0.30 \mathrm{mmol})$ in the presence of $\mathrm{Pd}(\mathrm{OAc})_{2}(3.4 \mathrm{mg}, 0.015 \mathrm{mmol})$, and 1-fluoro-2,4,6-trimethylpyridinium triflate (130.2 $\left.\mathrm{mg}, 0.45 \mathrm{mmol}\right)$ in acetic acid $(1 \mathrm{~mL})$, to afford a $1: 1.3$ isomeric mixture of $(\mathbf{1 7} \mathbf{a}+\mathbf{1 7} \mathbf{b})$ which was separated upon purification by column chromatography ( $n$-hexane-EtOAc 80:20).

Product $( \pm)-\mathbf{1 7 a}$ was obtained as yellow oil; yield: $22.6 \mathrm{mg}(31 \%) .{ }^{1} \mathrm{H}$ NMR $\left(300 \mathrm{MHz}, \mathrm{CDCl}_{3}\right): \delta$ $8.53(\mathrm{~d}, J=4.5 \mathrm{~Hz}, 1 \mathrm{H}), 7.70-7.65(\mathrm{~m}, 2 \mathrm{H}), 7.65(\mathrm{~d}, J=16.0 \mathrm{~Hz}, 1 \mathrm{H}), 7.35(\mathrm{~m}, 1 \mathrm{H}), 7.15(\mathrm{~m}, 1 \mathrm{H}), 7.00$ $(\mathrm{d}, J=7.5 \mathrm{~Hz}, 1 \mathrm{H}), 6.90(\mathrm{~m}, 1 \mathrm{H}), 6.47(\mathrm{~d}, J=16.0 \mathrm{~Hz}, 1 \mathrm{H}), 4.84(\mathrm{dd}, J=6.0,9.0 \mathrm{~Hz}, 1 \mathrm{H}), 4.14(\mathrm{t}, J=$ $6.7 \mathrm{~Hz}, 2 \mathrm{H}), 3.90(\mathrm{dc}, J=2.0,4.6 \mathrm{~Hz}, 2 \mathrm{H}), 3.32(\mathrm{dd}, J=9.0,14.5 \mathrm{~Hz}, 1 \mathrm{H}), 3.06(\mathrm{dd}, J=9.0,14.5 \mathrm{~Hz}$, 1H), $3.00(\mathrm{~m}, 3 \mathrm{H}), 1.62(\mathrm{~m}, 2 \mathrm{H}), 1.36(\mathrm{~m}, 2 \mathrm{H}) ; 1.01(\mathrm{t}, J=7.1 \mathrm{~Hz}, 3 \mathrm{H}), 0.90(\mathrm{t}, J=7.1 \mathrm{~Hz}, 3 \mathrm{H})$. 
Revised manuscript. Article published in J. Org. Chem. 2015, 80, 3321-3331 (DOI: $10.1021 / j 0502912 \mathrm{~m}$ )

${ }^{13} \mathrm{C}\left\{{ }^{1} \mathrm{H}\right\}$ NMR $\left(75 \mathrm{MHz}, \mathrm{CDCl}_{3}\right): \delta 169.5,166.9,157.4,149.8,138.4,137.6,134.9,130.5\left(\mathrm{~d}, J_{C-F}=13.0\right.$ $\mathrm{Hz}), 126.3,126.2\left(\mathrm{~d}, J_{C-F}=23.4 \mathrm{~Hz}\right), 125.5,125.3,121.9,115.1,114.1,64.6,61.4,60.6,32.7,31.5$, 30.7, 19.2, 13.9, 13.7. ${ }^{19} \mathrm{~F}\left\{{ }^{1} \mathrm{H}\right\}$ NMR (282 $\left.\mathrm{MHz}, \mathrm{CDCl}_{3}\right): \delta$-110.2. ESI-HRMS: Calcd for $\mathrm{C}_{24} \mathrm{H}_{30} \mathrm{FN}_{2} \mathrm{O}_{6} \mathrm{~S}[\mathrm{M}+\mathrm{H}]^{+}:$493.1803. Found: 493.1808.

Product $( \pm)-\mathbf{1 7 b}$ was obtained as yellow oil; yield: $30.5 \mathrm{mg}(41 \%) .{ }^{1} \mathrm{H}$ NMR $\left(300 \mathrm{MHz}, \mathrm{CDCl}_{3}\right): \delta$ $8.55(\mathrm{~m}, 1 \mathrm{H}), 7.87(\mathrm{~d}, J=15.8 \mathrm{~Hz}, 1 \mathrm{H}), 7.76-7.74(\mathrm{~m}, 2 \mathrm{H}), 7.45(\mathrm{dd}, J=6.0,8.4 \mathrm{~Hz}, 1 \mathrm{H}), 7.37(\mathrm{~m}, 1 \mathrm{H})$ 6.90-6.83 (m, 2H), $6.24(\mathrm{~d}, J=15.8 \mathrm{~Hz}, 1 \mathrm{H}), 4.84(\mathrm{dd}, J=4.7,8.6 \mathrm{~Hz}, 1 \mathrm{H}), 4.14(\mathrm{t}, J=6.5 \mathrm{~Hz}, 2 \mathrm{H})$, $3.91(\mathrm{c}, J=6.0 \mathrm{~Hz}, 2 \mathrm{H}), 3.30(\mathrm{dd}, J=6.7,14.3 \mathrm{~Hz}, 1 \mathrm{H}), 3.06(\mathrm{~m}, 1 \mathrm{H}), 3.00(\mathrm{~s}, 3 \mathrm{H}), 1.62(\mathrm{~m}, 2 \mathrm{H}), 1.35$ $(\mathrm{m}, 2 \mathrm{H}) ; 1.01(\mathrm{t}, J=7.1 \mathrm{~Hz}, 3 \mathrm{H}), 0.88(\mathrm{t}, J=7.1 \mathrm{~Hz}, 3 \mathrm{H}) .{ }^{13} \mathrm{C}\left\{{ }^{1} \mathrm{H}\right\} \mathrm{NMR}\left(75 \mathrm{MHz}, \mathrm{CDCl}_{3}\right): \delta 169.5$, $166.7,162.4,157.3,149.9,140.1,138.5,137.7,130.1,128.9\left(\mathrm{~d}, J_{C-F}=8.3 \mathrm{~Hz}\right), 126.4,122.0,120.6(\mathrm{~d}$, $\left.J_{C-F}=1.9 \mathrm{~Hz}\right), 117.5\left(\mathrm{~d}, J_{C-F}=22.0 \mathrm{~Hz}\right), 114.8\left(\mathrm{~d}, J_{C-F}=22.0 \mathrm{~Hz}\right), 64.6,61.5,60.7,32.6,31.5,30.8$, 19.2, 13.9, 13.8. ${ }^{19} \mathrm{~F}\left\{{ }^{1} \mathrm{H}\right\}$ NMR $\left(282 \mathrm{MHz}, \mathrm{CDCl}_{3}\right): \delta-110.5$. ESI-HRMS: Calcd for $\mathrm{C}_{24} \mathrm{H}_{30} \mathrm{FN}_{2} \mathrm{O}_{6} \mathrm{~S}$ $[\mathrm{M}+\mathrm{H}]^{+}:$493.1803. Found: 493.1800 .

$( \pm)-(E)$-Butyl 3-(3-(3-ethoxy-2-( $N$-methylpyridine-2-sulfonamido)-3-oxopropyl)naphthalen-2yl)acrylate $[( \pm)-18]$. Following the typical procedure, sulfonamide $( \pm)$-XXVI $(59.8 \mathrm{mg}, 0.15 \mathrm{mmol})$ reacted with butyl acrylate $(43.0 \mu \mathrm{L}, 0.30 \mathrm{mmol})$ in the presence of $\mathrm{Pd}(\mathrm{OAc})_{2}(3.4 \mathrm{mg}, 0.015 \mathrm{mmol})$, and 1-fluoro-2,4,6-trimethylpyridinium triflate $(130.2 \mathrm{mg}, 0.45 \mathrm{mmol})$ in acetic acid $(1 \mathrm{~mL})$, to afford, after purification by column chromatography ( $n$-hexane-EtOAc 80:20), product $( \pm)$-18 as yellow oil; yield: $40.9 \mathrm{mg}(52 \%)$.

${ }^{1} \mathrm{H}$ NMR (300 MHz, $\left.\mathrm{CDCl}_{3}\right): \delta 8.47(\mathrm{~d}, J=4.2 \mathrm{~Hz}, 1 \mathrm{H}), 8.16-8.11(\mathrm{~m}, 2 \mathrm{H}), 7.75(\mathrm{~d}, J=6.7 \mathrm{~Hz}, 1 \mathrm{H})$, 7.69-7.63 (m, 2H), 7.56-7.45 (m, 3H), $7.30(\mathrm{~m}, 1 \mathrm{H}), 6.32(\mathrm{~d}, J=15.7 \mathrm{~Hz}, 1 \mathrm{H}), 5.01(\mathrm{t}, J=7.4 \mathrm{~Hz}, 1 \mathrm{H})$, $4.15(\mathrm{t}, J=6.7 \mathrm{~Hz}, 2 \mathrm{H}), 3.90-3.75(\mathrm{~m}, 3 \mathrm{H}), 3.56(\mathrm{dd}, J=7.6,14.5 \mathrm{~Hz}, 1 \mathrm{H}), 3.10(\mathrm{~s}, 3 \mathrm{H}), 1.62(\mathrm{~m}, 2 \mathrm{H})$, $1.38(\mathrm{~m}, 2 \mathrm{H}), 0.90(\mathrm{~m}, 6 \mathrm{H}) .{ }^{13} \mathrm{C}\left\{{ }^{1} \mathrm{H}\right\}$ NMR $\left(75 \mathrm{MHz}, \mathrm{CDCl}_{3}\right): \delta$ 169.8, 166.9, 157.2, 149.7, 142.1, $137.6,134.3,133.0,132.1,131.6,128.9,128.3,127.3,126.8,126.3,124.4,123.6,121.9,120.9,64.5$, 
Revised manuscript. Article published in J. Org. Chem. 2015, 80, 3321-3331 (DOI: 10.1021/jo502912m)

61.3, 60.4, 31.8, 30.8, 28.8, 19.2, 13.8. ESI-HRMS: Calcd for $\mathrm{C}_{28} \mathrm{H}_{33} \mathrm{~N}_{2} \mathrm{O} 66 \mathrm{~S}[\mathrm{M}+\mathrm{H}]^{+}: 525.2054$. Found: 525.2059

( \pm )-(E)-Butyl 3-(1-(3-ethoxy-2-( $N$-methylpyridine-2-sulfonamido)-3-oxopropyl) naphthalen-2yl)acrylate $[( \pm)-19]$. Following the typical procedure, sulfonamide compound $( \pm)$-XXV (59.8 mg, 0.15 mmol) reacted with butyl acrylate $(43.0 \mu \mathrm{L}, 0.30 \mathrm{mmol})$ in the presence of $\mathrm{Pd}(\mathrm{OAc})_{2}(3.4 \mathrm{mg}, 0.015$ mmol), and 1-fluoro-2,4,6-trimethylpyridinium triflate $(130.2 \mathrm{mg}, 0.45 \mathrm{mmol})$ in acetic acid (1 mL), to afford, after purification by column chromatography ( $n$-hexane-EtOAc 80:20), product $( \pm)$-19 as yellow oil; yield: $54.3 \mathrm{mg}(69 \%)$.

${ }^{1} \mathrm{H}$ NMR (300 MHz, CDCl3): $\delta 8.33(\mathrm{~d}, J=4.5 \mathrm{~Hz}, 1 \mathrm{H}), 8.03(\mathrm{~d}, J=15.7 \mathrm{~Hz}, 1 \mathrm{H}), 7.89(\mathrm{~s}, 1 \mathrm{H}), 7.70$ (m, 1H), $7.61(\mathrm{~m}, 1 \mathrm{H}), 7.55-7.35(\mathrm{~m}, 5 \mathrm{H}), 7.07(\mathrm{~m}, 1 \mathrm{H}), 6.42(\mathrm{~d}, J=15.7 \mathrm{~Hz}, 1 \mathrm{H}), 4.95(\mathrm{dd}, J=6.0,9.1$ $\mathrm{Hz}, 1 \mathrm{H}), 4.27(\mathrm{t}, J=6.7 \mathrm{~Hz}, 2 \mathrm{H}), 3.94(\mathrm{dc}, J=1.5,7.0 \mathrm{~Hz}, 2 \mathrm{H}), 3.47(\mathrm{dd}, J=6.0,14.5 \mathrm{~Hz}, 1 \mathrm{H}), 3.15$ $(\mathrm{dd}, J=6.0,14.5 \mathrm{~Hz}, 1 \mathrm{H}), 3.10(\mathrm{~m}, 3 \mathrm{H}), 1.62(\mathrm{~m}, 2 \mathrm{H}), 1.40(\mathrm{~m}, 2 \mathrm{H}), 1.01(\mathrm{t}, J=7.1 \mathrm{~Hz}, 3 \mathrm{H}), 0.90(\mathrm{t}, J$ $=7.1 \mathrm{~Hz}, 3 \mathrm{H}) .{ }^{13} \mathrm{C}\left\{{ }^{1} \mathrm{H}\right\} \operatorname{NMR}\left(75 \mathrm{MHz}, \mathrm{CDCl}_{3}\right): \delta=169.8,166.7,157.5,149.5,141.6,137.3,133.8$, $132.7,132.4,132.3,129.3,128.0,127.5,127.0,126.9,126.4,125.9,121.5,121.4,64.6,61.4,60.7,33.0$, 31.5, 30.8, 19.2, 14.0, 13.8. ESI-HRMS: Calcd for $\mathrm{C}_{28} \mathrm{H}_{33} \mathrm{~N}_{2} \mathrm{O}_{6} \mathrm{~S}[\mathrm{M}+\mathrm{H}]^{+}$: 525.2054. Found: 525.2059. (E)-Butyl 3-(2-((1R,2S)-1-methoxy-2-( $N$-methylpyridine-2-sulfonamido)propyl)phenyl)acrylate [(-)-21]. Following the typical procedure, $(-)-20(48.1 \mathrm{mg}, 0.15 \mathrm{mmol})$ reacted with butyl acrylate $(21.5$ $\mu \mathrm{L}, 0.15 \mathrm{mmol})$ in the presence of $\mathrm{Pd}(\mathrm{OAc})_{2}(3.4 \mathrm{mg}, 0.015 \mathrm{mmol})$, and 1-fluoro-2,4,6trimethylpyridinium triflate $(130.2 \mathrm{mg}, 0.45 \mathrm{mmol})$ in acetic acid $(1 \mathrm{~mL})$, to afford, after purification by column chromatography ( $n$-hexane-EtOAc 80:20), product (-)-21 as colorless oil; yield : $51.4 \mathrm{mg}$ $(74 \%)$.

$[\alpha]_{\mathrm{D}}{ }^{298}=-73.8\left(c\right.$ 0.5, $\left.\mathrm{CHCl}_{3}\right) .{ }^{1} \mathrm{H} \mathrm{NMR}\left(300 \mathrm{MHz}, \mathrm{CDCl}_{3}\right): \delta 8.77-8.63(\mathrm{~m}, 1 \mathrm{H}), 8.25(\mathrm{~d}, J=15.7 \mathrm{~Hz}$, 1H), $8.05-7.93(\mathrm{~m}, 1 \mathrm{H}), 7.87(\mathrm{td}, J=7.7,1.7 \mathrm{~Hz}, 1 \mathrm{H}), 7.58(\mathrm{~d}, J=7.7 \mathrm{~Hz}, 1 \mathrm{H}), 7.52-7.25(\mathrm{~m}, 4 \mathrm{H})$, $6.38(\mathrm{~d}, J=15.7 \mathrm{~Hz}, 1 \mathrm{H}), 4.85(\mathrm{~d}, J=4.3 \mathrm{~Hz}, 1 \mathrm{H}), 4.23(\mathrm{dt}, J=8.7,5.5 \mathrm{~Hz}, 2 \mathrm{H}), 3.25(\mathrm{~s}, 3 \mathrm{H}), 3.07(\mathrm{~s}$, 
Revised manuscript. Article published in J. Org. Chem. 2015, 80, 3321-3331 (DOI: $10.1021 / j o 502912 \mathrm{~m}$ )

$3 \mathrm{H}), 1.87-1.60(\mathrm{~m}, 3 \mathrm{H}), 1.03(\mathrm{~d}, J=7.0 \mathrm{~Hz}, 3 \mathrm{H}), 0.98(\mathrm{t}, J=7.4 \mathrm{~Hz}, 3 \mathrm{H}) .{ }^{13} \mathrm{C}\left\{{ }^{1} \mathrm{H}\right\} \mathrm{NMR}(75 \mathrm{MHz}$, $\left.\mathrm{CDCl}_{3}\right): \delta 166.7,158.0,149.8,141.3,137.9,137.7,133.8,129.6,127.9,127.4,126.8,126.1,122.5$, 121.2, 84.5, 64.4, 57.2, 57.1, 31.3, 30.7, 19.1, 13.7, 11.1. ESI-HRMS: Calcd for $\mathrm{C}_{23} \mathrm{H}_{31} \mathrm{~N}_{2} \mathrm{O}_{5} \mathrm{~S}[\mathrm{M}+\mathrm{H}]^{+}$: 447.1948. Found: 447.1952.

$N-((1 R, 2 S)-1-M e t h o x y-1-(2-((E)-2-($ phenylsulfonyl)vinyl)phenyl)propan-2-yl)- $N$-methylpyridine2-sulfonamide [(-)-22]. Following the typical procedure, (-)-20 (48.1 $\mathrm{mg}, 0.15 \mathrm{mmol})$ reacted with phenyl vinyl sulfone $(25.2 \mathrm{mg}, 0.15 \mathrm{mmol})$ in the presence of $\mathrm{Pd}(\mathrm{OAc})_{2}(3.4 \mathrm{mg}, 0.015 \mathrm{mmol})$, and 1fluoro-2,4,6-trimethylpyridinium triflate $(130.2 \mathrm{mg}, 0.45 \mathrm{mmol})$ in acetic acid $(1 \mathrm{~mL})$, to afford, after purification by column chromatography ( $n$-hexane-EtOAc 80:20), product (-)-22 as colorless oil; yield : $56.9 \mathrm{mg}(78 \%)$.

$[\alpha]^{298}=-63.51\left(c \quad 0.4, \mathrm{CHCl}_{3}\right) .{ }^{1} \mathrm{H}$ NMR $\left(300 \mathrm{MHz}, \mathrm{CDCl}_{3}\right): \delta 8.84-8.60(\mathrm{~m}, 1 \mathrm{H}), 8.27(\mathrm{~d}, J=15.1$ $\mathrm{Hz}, 1 \mathrm{H}), 8.15-8.03(\mathrm{~m}, 2 \mathrm{H}), 8.05-7.95(\mathrm{~m}, 1 \mathrm{H}), 7.90(\mathrm{td}, J=7.7,1.8 \mathrm{~Hz}, 1 \mathrm{H}), 7.73-7.35(\mathrm{~m}, 7 \mathrm{H})$, $7.31(\mathrm{td}, J=7.2,1.9 \mathrm{~Hz}, 1 \mathrm{H}), 6.85(\mathrm{~d}, J=15.2 \mathrm{~Hz}, 1 \mathrm{H}), 4.84(\mathrm{~d}, J=4.7 \mathrm{~Hz}, 1 \mathrm{H}), 4.18(\mathrm{tt}, J=7.0,3.5$ $\mathrm{Hz}, 1 \mathrm{H}), 3.21(\mathrm{~s}, 3 \mathrm{H}), 3.04(\mathrm{~s}, 3 \mathrm{H}), 1.05(\mathrm{~d}, J=7.0 \mathrm{~Hz}, 3 \mathrm{H}) .{ }^{13} \mathrm{C}\left\{{ }^{1} \mathrm{H}\right\} \mathrm{NMR}\left(75 \mathrm{MHz}, \mathrm{CDCl}_{3}\right): \delta 157.8$, $149.9,140.5,139.6,138.7,137.8,133.2,131.6,130.6,129.7,129.2,128.2,128.1,128.0,127.4,126.3$, 122.6, 84.7, 57.5, 57.2, 31.2, 11.5. ESI-HRMS: Calcd for $\mathrm{C}_{24} \mathrm{H}_{27} \mathrm{~N}_{2} \mathrm{O}_{5} \mathrm{~S}_{2}[\mathrm{M}+\mathrm{H}]^{+}:$487.1356. Found: 487.1351.

Typical procedure for the $\mathrm{Zn}$-promoted reductive $N$-desulfonylation: synthesis of $\left(1 R^{*}, 3 S^{*}\right)$ Ethyl 1-(2-butoxy-2-oxoethyl)-2-methyl-1,2,3,4-tetrahydroisoquinoline-3-carboxylate $[( \pm)-23)$. A suspension of ( \pm )-2 (47.5 mg, $0.1 \mathrm{mmol})$ and Zn powder $(346 \mathrm{mg}, 5 \mathrm{mmol})$ in a 1:1 mixture of THF and sat. aq $\mathrm{NH}_{4} \mathrm{Cl}$ solution $\left(2 \mathrm{~mL}\right.$ ) was stirred at $65{ }^{\circ} \mathrm{C}$ until consumption of the starting material (TLC monitoring, $72 \mathrm{~h})$. The mixture was diluted with EtOAc $(15 \mathrm{~mL})$ and filtered over a plug of Celite to remove the $\mathrm{Zn}$ (washed with EtOAc). The combined filtrate was washed with brine $(10 \mathrm{~mL})$ and the combined organic phase was dried $\left(\mathrm{MgSO}_{4}\right)$ and concentrated to dryness. The residue was purified by 
Revised manuscript. Article published in J. Org. Chem. 2015, 80, 3321-3331 (DOI: $10.1021 / j 0502912 \mathrm{~m}$ )

flash chromatography ( $n$-hexane-EtOAc 9:1) to afford the tetrahydroisoquinoline derivative ( \pm )-23 as yellow oil (80:20 mixture of diastereomers); yield: $22.4 \mathrm{mg}(71 \%)$.

${ }^{1} \mathrm{H}$ NMR $\left(300 \mathrm{MHz}, \mathrm{CDCl}_{3}\right): \delta 7.10-7.00(\mathrm{~m}, 4 \mathrm{H}), 4.31(\mathrm{t}, J=6.8 \mathrm{~Hz}, 1 \mathrm{H}), 4.14(\mathrm{~m}, 1 \mathrm{H}), 4.10-3.95$ (m, 3H), $3.77(\mathrm{dd}, J=5.6,7.6 \mathrm{~Hz}, 1 \mathrm{H}), 3.04(\mathrm{dd}, J=16.3,7.5 \mathrm{~Hz}, 1 \mathrm{H}), 2.93(\mathrm{dd}, J=16.5,5.5 \mathrm{~Hz}, 1 \mathrm{H})$, $2.73(\mathrm{dd}, J=14.9,6.5 \mathrm{~Hz}, 1 \mathrm{H}), 2.63(\mathrm{dd}, J=15.0,6.2 \mathrm{~Hz}, 1 \mathrm{H}), 2.41(\mathrm{~s}, 3 \mathrm{H}), 1.49(\mathrm{~m}, 2 \mathrm{H}), 1.23(\mathrm{~m}$, 2H), $1.12(\mathrm{t}, J=7.2 \mathrm{~Hz}, 3 \mathrm{H}), 0.84(\mathrm{~m}, 3 \mathrm{H}) .{ }^{13} \mathrm{C}\left\{{ }^{1} \mathrm{H}\right\}$ NMR $\left(75 \mathrm{MHz}, \mathrm{CDCl}_{3}\right): \delta 172.6,171.7,137.0$, $132.2,128.8,126.9,126.4,126.3,64.3,60.5,60.3,58.4,42.1,39.1,28.5,27.5,19.1,14.2,13.7$. ESIHRMS: Calcd for $\mathrm{C}_{19} \mathrm{H}_{28} \mathrm{NO}_{4}[\mathrm{M}+\mathrm{H}]^{+}:$334.2013. Found: 334.2016 .

\section{$(1 S, 3 S)-$ Ethyl}

2-methyl-1-((phenylsulfonyl)methyl)-1,2,3,4-tetrahydroiso-quinoline-3-

carboxylate $[(+)-24]$. Following the typical procedure, $(S)-5(51.4 \mathrm{mg}, 0.1 \mathrm{mmol})$ and Zn powder (346 $\mathrm{mg}, 5 \mathrm{mmol})$ to afford, after flash chromatography ( $n$-hexane-EtOAc 9:1), product (+)-24 as yellow oil (80:20 mixture of diastereomers); yield: $26.2 \mathrm{mg}$ (74\%) (95\% ee for a pure sample of anti-(+)-24).

$[\alpha]_{\mathrm{D}}^{298}=+38.88\left(c\right.$ 0.18, $\left.\mathrm{CHCl}_{3}\right) .{ }^{1} \mathrm{H}$ NMR $\left(300 \mathrm{MHz}, \mathrm{CDCl}_{3}\right): \delta$ 8.03-7.97 $(\mathrm{m}, 2 \mathrm{H}), 7.70-7.55(\mathrm{~m}$, 3H), 7.26-7.08 (m, 4H), $4.53(\mathrm{~m}, 1 \mathrm{H}), 4.09(\mathrm{c}, J=7.1 \mathrm{~Hz}, 2 \mathrm{H}), 3.75-3.64(\mathrm{~m}, 2 \mathrm{H}), 3.50(\mathrm{dd}, J=4.0$, $15.0 \mathrm{~Hz}, 1 \mathrm{H}), 3.02(\mathrm{dd}, J=16.5,7.8 \mathrm{~Hz}, 1 \mathrm{H}), 2.95(\mathrm{dd}, J=6.1,17.1 \mathrm{~Hz}, 1 \mathrm{H}), 2.36(\mathrm{~s}, 3 \mathrm{H}), 1.20(\mathrm{t}, J=$ $7.1 \mathrm{~Hz}, 3 \mathrm{H}) .{ }^{13} \mathrm{C}\left\{{ }^{1} \mathrm{H}\right\} \mathrm{NMR}\left(75 \mathrm{MHz}, \mathrm{CDCl}_{3}\right): \delta 171.7,140.5,135.2,133.3,132.5,129.0,128.9,128.2$, 127.4, 127.1, 127.0, 63.0, 60.6, 58.8, 57.6, 38.3, 27.5, 14.2. ESI-HRMS: Calcd for $\mathrm{C}_{20} \mathrm{H}_{24} \mathrm{NO}_{4} \mathrm{~S}$ $[\mathrm{M}+\mathrm{H}]^{+}:$374.1421. Found: 374.1427. HPLC: Daicel Chiralpak IA, hexane-isopropanol 90-10, flow rate $0.7 \mathrm{~mL} / \min (\ddot{\mathrm{e}}=254 \mathrm{~nm}), t_{\mathrm{R}}: 22.7 \min (1 R, 3 R)-\mathbf{2 4}$ and $34.7 \min (1 S, 3 S)-\mathbf{2 4}$.

\section{ASSOCIATED CONTENT}

Experimental details as well as spectroscopic and analytical data for the synthesis of the starting sulfomamides. HPLC traces for enantienriched compounds. Copies of ${ }^{1} \mathrm{H}$ NMR and ${ }^{13} \mathrm{C}$ NMR of new compounds. Copies of NOE experiments for relative stereochemistry determination of compounds $\mathbf{2 3}$ 
Revised manuscript. Article published in J. Org. Chem. 2015, 80, 3321-3331 (DOI: $10.1021 / j 0502912 \mathrm{~m}$ )

and 24. Catalyst system optimization studies. This material is available free of charge via the Internet at http://pubs.acs.org.

\section{AUTHOR INFORMATION}

\section{Corresponding Authors}

ramon.gomez@uam.es; juancarlos.carretero@uam.es.

ACKNOWLEDGMENT. We thank Spanish Government (MINECO, CTQ2012-35790, CTQ201122589 and CTQ2013-40855-R) and Aragón government (research groups E40 and E97) for financial support. E. L. thanks Aragón government for a $\mathrm{PhD}$ grant.

\section{References}

(1) For general recent reviews on C-H functionalization: (a) Godula, K.; Sames, D. Science 2006, 312, 67. (b) Bruckl, T.; Baxter, R. D.; Ishihara Y.; Baran, P. S. Acc. Chem. Res., 2012, 45, 826. (c) Newhouse, T.; Baran, P. S. Angew. Chem., Int. Ed., 2011, 50, 3362. (d) Engle, K. M.; Mei, T.-S.; Wasa, M.; Yu, J.-Q. Acc. Chem. Res. 2012, 45, 788. (e) Kuhl, N.; Hopkinson, M. N.; Wencel-Delord, J.; Glorius, F. Angew. Chem. Int. Ed. 2012, 51, 10236. (f) Arockiam, P. B.; Bruneau, C.; Dixneuf, P. H. Chem. Rev. 2012, 112, 5879. (g) Yamaguchi, J.; Yamaguchi, A. D.; Itami, K. Angew. Chem. Int. Ed. 2012, 51, 8960. (h) Yu, D.-G.; Li, B.-J.; Shi, Z.-J. Tetrahedron 2012, 68, 5130. (i) Mousseau, J. J.; Charette, A. B. Acc. Chem. Res. 2013, 46, 412. (j) Wencel-Delord, J.; Glorius, F. Nat. Chem. 2013, 5, 369. (k) Sharma, A.; Vacchani, D.; Van der Eycken, E. Chem. Eur. J. 2013, 19, 1158. For a review on stoichiometric and catalytic cyclometalation strategies: (1) Cuesta, L.; Urriolabeitia, E. P. Comm. Inorg. Chem., 2012, 33, 55. 
Revised manuscript. Article published in J. Org. Chem. 2015, 80, 3321-3331 (DOI: $10.1021 / j 0502912 \mathrm{~m}$ )

(2) For a review on Pd-catalyzed remote $\mathrm{C}-\mathrm{H}$ functionalization: Franzoni, I.; Mazet, C. Org. Biomol. Chem. 2014, 12, 233.

(3) For reviews on removable directing groups in $\mathrm{C}-\mathrm{H}$ activation, see: (a) Rousseau, G.; Breit, B. Angew. Chem. Int. Ed. 2011, 50, 2450. (b) Wang, C.; Huang, Y. Synlett 2013, 24, 145. (c) Rouquet, G.; Chatani, N. Angew. Chem. Int. Ed. 2013, 52, 11726.

(4) For the wide range significance of $\alpha$-amino acids, see: Amino Acids, Peptides and Proteins in Organic Chemistry, Vol 1-5 (Series Ed.: A. B. Hughes), Wiley-VCH, Weinheim, 2009-2013.

(5) For a general review on $\mathrm{C}-\mathrm{H}$ functionalization in the synthesis of amino acids and peptides, see: Noisier, A. F. M.; Brimble, M. A. Chem. Rev. 2014, 114, 8775.

(6) For representative examples of functionalization of $\alpha$-amino acid derivatives using a stoichiometric amount of metal: (a) Vicente, J.; Saura-Llamas, I.; García-López, J. A.; Bautista, D. Organometallics 2009, 28, 448. (b) Arnau, P.; Nieto, S.; Serrano, E.; Navarro, R.; Soler, T.; Cativiela, C.; Urriolabeitia, E. P. Inorg. Chem. 2009, 48, 11963. (c) Vicente, J.; Saura-Llamas, I.; García-López, J. A.; Bautista, D. Organometallics 2010, 29, 4320. (d) Nieto, S.; Sayago, F. J.; Laborda, P.; Soler, T.; Cativiela, C.; Urriolabeitia, E. P. Tetrahedron 2011, 67, 4185. (e) García-López, J. A.; Saura-Llamas, I.; McGrady, J. E.; Bautista, D.; Vicente, J. Organometallics 2012, 31, 8333. (f) Oliva-Madrid, M. J.; García-López, J. A.; Saura-Llamas, I.; Bautista, D.; Vicente, J. Organometallics 2012, 31, 3647. (g) Laga, E.; Cativiela, C.; Urriolabeitia, E. P. Beilstein J. Org. Chem. 2012, 8, 1569. (h) Laga, E.; GarcíaMontero, A.; Sayago, F. J.; Soler, T.; Moncho, S.; Cativiela, C.; Martinez, M.; Urriolabeitia, E. P. Chem. Eur. J. 2013, 19, 17398.

(7) For catalytic $\mathrm{C}\left(\mathrm{sp}^{2}\right)-\mathrm{H}$ functionalization of aromatic $\alpha$-amino acid derivatives, see: (a) Li, J. J.; Mei, T.-S.; Yu, J.-Q. Angew. Chem. Int. Ed. 2008, 47, 6452. (b) Mei, T.-S.; Wang, X.; Yu, J.-Q. J. Am. 
Revised manuscript. Article published in J. Org. Chem. 2015, 80, 3321-3331 (DOI: $10.1021 / j 0502912 \mathrm{~m}$ )

Chem. Soc. 2009, 131, 10806. (c) Meyer, F.-M.; Liras, S.; Guzman-Perez, A.; Perreault, C.; Bian, J.; James, K. Org. Lett. 2010, 12, 3870. (d) Vickers, C. J.; Mei, T.-S.; Yu, J.-Q. Org. Lett. 2010, 12, 2511. (e) López, B.; Rodriguez, A.; Santos, D.; Albert, J.; Ariza, X.; Garcia, J.; Granell, J. Chem. Commun., 2011, 47, 1054. (f) He, G.; Zhao, Y.; Zhang, S.; Lu, C.; Chen, G. J. Am. Chem. Soc. 2012, 134, 3. (g) Albert, J.; Ariza, X.; Calvet, T.; Font-Bardia, M.; Garcia, J.; Granell, J.; Lamela, A.; López, B.; Martinez, M.; Ortega, L.; Rodriguez, A.; Santos, D. Organometallics 2013, 32, 649. (h) Mei, T.-S.; Leow, D.; Xiao, H.; Laforteza, B. N.; Yu, J.-Q. Org. Lett. 2013, 15, 3058. (i) Zhu, Y.; Bauer, M.; Ploog, J.; Ackermann, L. Chem. Eur. J. 2014, 20, 13099.

(8) For the catalytic activation of $\mathrm{C}\left(\mathrm{sp}^{3}\right)-\mathrm{H}$ bonds of $\alpha$-amino acid derivatives, see: (a) Dangel, B. D.; Johnson, J. A.; Sames, D. J. Am. Chem. Soc. 2001, 123, 8149. (b) Reddy, B. V. S.; Reddy, L. R.; Corey, E. J. Org. Lett. 2006, 8, 3391. (c) Zhao, L.; Baslé , O.; Li, C.-J. Proc. Natl. Acad. Sci. USA 2009, 106, 4106. (d) Shabashov, D.; Daugulis, O. J. Am. Chem. Soc. 2010, 132, 3965. (e) He, G.; Chen, G. Angew. Chem. Int. Ed. 2011, 50, 5192. (f) Tran, L. D.; Daugulis, O. Angew. Chem. Int. Ed. 2012, 51, 5188. (g) Aspin, S.; Goutierre, A.-S.; Larini, P.; Jazzar, R.; Baudoin O. Angew. Chem. Int. Ed. 2012, 51, 10808. (h) Fan, M.; Ma, D. Angew. Chem. Int. Ed. 2013, 52, 12152. (i) Rodríguez, N.; Romero-Revilla, J. A.; Fernández-Ibáñez, M. A.; Carretero, J. C. Chem. Sci. 2013, 4, 175. (j) Zhang, S.-Y.; He, G.; Nack, W. A.; Zhao, Y.; Li, Q.; Chen, G. J. Am. Chem. Soc. 2013, 135, 2124. (k) Chen, K.; Hu, F.; Zhang S.Q.; Shi, B.-F. Chem. Sci., 2013, 4, 3906. (1) McNally, A.; Haffemayer, B.; Collins, B. S. L.; Gaunt, M. J. Nature 2014, 150, 129. (m) Li, Q.; Zhang, S.-Y.; He, G.; Nack, W. A.; Chen, G. Adv. Synth. Catal. 2014, 356, 1544. (n) Chan, K. S. L.; Wasa, M.; Chu, L.; Laforteza, B. N.; Miura, M.; Yu, J.-Q. Nat. Chem. 2014, 6, 146. (o) Chen, K.; Zhang, S.-Q.; Xu, J.-W.; Hu, F.; Shi, B.-F. Chem.Commun. 2014, 50,13924. (p) Zhu, R.-Y.; He, J.; Wang, X.-C.; Yu, J.-Q. J. Am. Chem. Soc. 2014, 136, 13194. (q) Gong, W.; Zhang, G.; Liu, T.; Giri, R.; Yu J.-Q. J. Am. Chem. Soc. 2014, 136, 16940. (r) He, J.; Li, S.; Deng, Y.; Fu, H.; Laforteza, B. N.; Spangler, J. E.; Homs, A.; Yu J.-Q. Science 2014, 343, 1216. (s) Poveda, 
Revised manuscript. Article published in J. Org. Chem. 2015, 80, 3321-3331 (DOI: 10.1021/jo502912m)

A.; Alonso I.; Fernández-Ibáñez, M. A. Chem. Sci., 2014, 5, 3873. (t) Zhang, L.-S.; Chen, G.; Wang, X.; Guo, Q.-Y.; Zhang, X.-S.; Pan, F.; Chen, K.; Shi, Z.-J. Angew. Chem. Int. Ed. 2014, 53, 3899. (u) Chen K.; Shi B.-F. Angew. Chem. Int. Ed. 2014, 53, 11950. See also ref. $7 \mathrm{f}$.

(9) For examples of $\mathrm{C}-\mathrm{H}$ olefination, followed by $\mathrm{N}$-functionalization, see: (a) Miura, M.; Tsuda, T.; Satoh, T.; Pivsa-Art, S.; Nomura, M. J. Org. Chem. 1998, 63, 5211. (b) Wasa, M.; Engle, K. M.; Yu, J.-Q. J. Am. Chem. Soc. 2010, 132, 3680. (c) Stowers, K. J.; Fortner, K. C.; Sanford, M. S. J. Am. Chem. Soc. 2011, 133, 6541. (d) Ji, X.; Huang, H.; Li, Y.; Chen, H.; Jiang, H. Angew. Chem. Int. Ed. 2012, 51, 7292. (e) Wang, N.-J.; Mei, S.-T.; Shuai, L.; Yuan, Y.; Wei, Y. Org. Lett. 2014, 16, 3040. (f) Cai, S.; Chen, C.; Shao, P.; Xi, C. Org. Lett. 2014, 16, 3142. (g) Manu Martínez, A.; Rodríguez, N.; Gómez Arrayás, R.; Carretero, J. C. Chem. Commun. 2014, 50, 6105. (h) Ye, X.; Shi, X. Org. Lett. 2014, 16, 4448. (i) Grigorjeva, L.; Daugulis, O. Org. Lett. 2014, 16, 4684. (j) Suzuki, C.; Morimoto, K.; Hirano, K.; Satoh, T.; Miura, M. Adv. Synth. Catal. 2014, 356, 1521. (k) Shi, Z.; Boultadakis-Arapinis, M.; Koester, D. C.; Glorius, F. Chem. Commun. 2014, 50, 2650. (1) Parthasarathy, K.; Bolm, C. Chem. Eur. J. 2014, 20, 4896.

(10) See, for instance: (a) Rauf, W.; Thompson, A. L.; Brown, J. M. Chem. Commun. 2009, 3874. (b) Engle, K. M.; Wang, D.-H.; Yu, J.-Q. Angew. Chem. Int. Ed. 2010, 49, 6169. (c) Li, G.; Leow, D.; Wan, L.; Yu, J.-Q. Angew. Chem. Int. Ed. 2013, 52, 1245. (d) Chan, L. Y.; Kim, S.; Ryu, T.; Lee, P. H. Chem. Commun. 2013, 49, 4682.

(11) For recent reviews on Fujiwara-Moritani reaction: (a) Le Bras, J.; Muzart, J. Chem. Rev. 2011, 111, 1170. (b) Yeung, C. S.; Dong, V. M. Chem. Rev. 2011, 111, 1215. (c) Kozhushkov, S. I.; Ackermann, L. Chem. Sci. 2013, 4, 886. (d) Wu, Y.; Wang, J.; Mao, F.; Kwong, F. Y. Chem. Asian J. 2014, 9, 26. (e) Zhou, L.; Lu, W. Chem. Eur. J. 2014, 20, 634. 
Revised manuscript. Article published in J. Org. Chem. 2015, 80, 3321-3331 (DOI: $10.1021 / j 0502912 \mathrm{~m}$ )

(12) For selected very recent examples: (a) Li, G.; Leow, D.; Wan, L.; Yu, J.-Q. Angew. Chem. Int. Ed. 2013, 52, 1245. (b) Huang, X.; Huang, J.; Du, C.; Zhang, X.; Song, F.; You, J. Angew. Chem. Int. Ed. 2013, 52, 12970. (c) Ma, W.; Ackermann, L. Chem. Eur. J. 2013, 19, 13925. (d) Wesch, T.; Leroux, F. R.; Colobert, F. Adv. Synth. Catal. 2013, 355, 2139. (e) Cheng, G.-J.; Yang, Y.-F.; Liu, P.; Chen, P.; Sun, T.-Y.; Li, G.; Zhang, X.; Houk, K. N.; Yu, J.-Q.; Wu Y.-D. J. Am. Chem. Soc. 2014, 136, 894. (f) Wang, B.; Shen, C.; Yao, J.; Yin, H.; Zhang, Y. Org. Lett. 2014, 16, 46. (g) Zhou, J.; Li, B.; Qian, Z.C.; Shi, B.-F. Adv. Synth. Catal. 2014, 356, 1038. (h) Yang, G.; Lindovska, P.; Zhu, D.; Kim, J.; Wang, P.; Tang, R.-Y.; Movassaghi, M.; Yu, J.-Q. J. Am. Chem. Soc. 2014, 136, 10807. (i) Vora, H. U.; Silvestri, A. P.; Engelin, C. J.; Yu, J.-Q. Angew. Chem. Int. Ed. 2014, 53, 268. (j) Fabry, D. C.; Zoller, J.; Raja, S.; Rueping, M. Angew. Chem. Int. Ed. 2014, 53, 10228. (k) Becker, P.; Priebbenow, D. L.; Pirwerdjan, R.; Bolm, C. Angew. Chem. Int. Ed. 2014, 53, 269. (1) She, Z.; Shi, Y.; Huang, Y.; Cheng, Y.; Song, F.; You, J. Chem. Commun. 2014, 50, 13914. (m) Deb, A.; Bag, S.; Kancherla, R.; Maiti, D. J. Am. Chem. Soc. 2014, 136, 13602. For aerobic oxidative coupling of arenes and olefins, see: (n) Babu, B. P.; Meng, X.; Bäckvall. J.-E. Chem. Eur. J. 2013, 19, 4140. (o) Gigant, N.; Bäckvall, J.-E. Org. Lett. 2014, 16, 1664.

(13) For previous examples on the use of $\mathrm{N}-\mathrm{SO}_{2} \mathrm{Py}$ directing group in $\mathrm{C}-\mathrm{H}$ functionalizations, see: (a) García-Rubia, A.; Gómez Arrayás, R.; Carretero, J. C. Angew. Chem. Int. Ed. 2009, 48, 6511. (b) García-Rubia, A.; Urones, B.; Gómez Arrayás, R.; Carretero, J. C. Chem. Eur. J. 2010, 16, 9676. (c) García-Rubia, A.; Urones, B.; Gómez Arrayás, R.; Carretero, J. C. Angew. Chem. Int. Ed. 2011, 50, 10927. (d) Urones, B.; Gómez Arrayás, R.; Carretero, J. C. Org. Lett. 2013, 15, 1120. (e) Urones, B.; Martínez, A. M.; Rodríguez, N.; Gómez Arrayás, R.; Carretero, J. C. Chem. Commun. 2013, 49, 11044. (f) Yan, Z.-L.; Chen, W.-L.; Gao, Y.-R.; Mao, S.; Zhang, Y.-L.; Wang, Y.-Q. Adv. Synth. Catal. 2014, 356, 1085. See also references $7 \mathrm{~h}, 8 \mathrm{i}$ and $8 \mathrm{~s}$. 
Revised manuscript. Article published in J. Org. Chem. 2015, 80, 3321-3331 (DOI: 10.1021/jo502912m)

(14) $\mathrm{N}$-Fluoro-2,4,6-trimethylpyridinium triflate has been used as oxidant in Pd-catalyzed C-H fluorination, trifluoromethylation and aminations, presumably through $\mathrm{Pd} \mathrm{d}^{\mathrm{I}} / \mathrm{Pd}^{\mathrm{IV}}$ mechanisms. For early success: (a) Wang, X.; Mei, T.-S.; Yu, J.-Q. J. Am. Chem. Soc. 2009, 131, 7520. For a review: (b) Engle, K. M.; Mei, T.-S.; Wang, X.; Yu. J.-Q. Angew. Chem. Int. Ed. 2011, 50, 1478.

(15) No products resulting from competing Heck-coupling were detected in the crude reaction mixtures of products $\mathbf{1 5}$ or 16. In agreement with this observation, no Heck-type side reaction was either observed in our previously reported alkenylation of aniline derivatives with electron-defficient alkenes in substrates holding a chloro or bromo-substitutent (see reference 13c).

(16) For a recent review on synthesis and biological significance of the 1,2,3,4tetrahydroisoquinoline-3-carboxylic acid (Tic) core, see: Kotha, S.; Deodhar, D.; Khedkar, P. Org. Biomol. Chem. 2014, 12, 9054.

(17) The relative configuration of the tetrahydroisoquinoline products has been established by ${ }^{1} \mathrm{H}$ NMR, mainly by NOE experiments (see Supporting Information for details). 